Aus der Klinik für Anästhesiologie

(Prof. Dr. med. K. Meißner)

der Medizinischen Fakultät der Universität Göttingen

\title{
Prospektive Analyse der Indikation von Notarzteinsätzen
}

\author{
INAUGURAL-DISSERTATION \\ zur Erlangung des Doktorgrades \\ der Medizinischen Fakultät der \\ Georg-August-Universität zu Göttingen
}

vorgelegt von

Mauricio Sebastian Arnoldt

aus

Cordoba, Argentinien

Göttingen 2021 
Dekan:

\section{Betreuungsausschuss}

Betreuer:

Ko-Betreuerin:

\section{Prüfungskommission}

Referent:

Ko-Referentin:

Promotor:

Datum der mündlichen Prüfung: 16.11.2021
PD Dr. med. Heise, D.

Prof. Dr. med. Blaschke, S.

PD Dr. med. Heise, D.

Prof. Dr. med. Blaschke, S.

Prof. Dr. med. Dressel, R. 
Hiermit erkläre ich, die Dissertation mit dem Titel "Prospektive Analyse der Indikation von Notarzteinsätzen" eigenständig angefertigt und keine anderen als die von mir angegebenen Quellen und Hilfsmittel verwendet zu haben.

Göttingen, den 21.02.2021

Mauricio Sebastian Arnoldt 


\section{Inhaltsverzeichnis}

Abbildungsverzeichnis .................................................................................. III

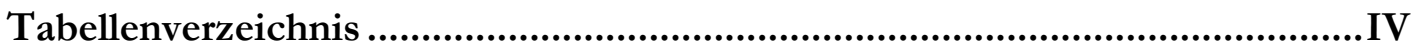

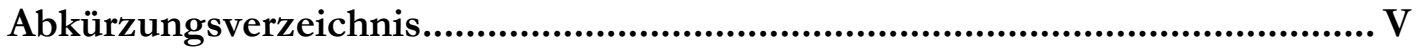

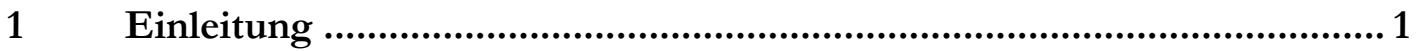

1.1 Die Rolle des Notarztes in der modernen präklinischen Notfallmedizin .......................... 1

1.2 Historische Entwicklung des ärztlich betriebenen Rettungsdienstes................................2

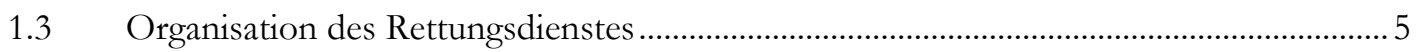

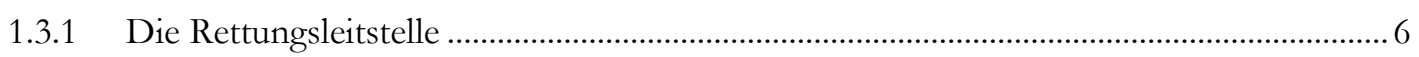

1.3.2 Die Einsatzmittel im Rettungsdienst........................................................................

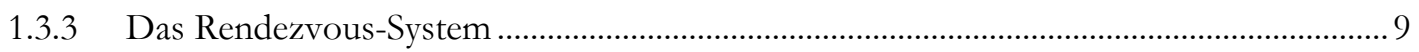

1.3.4 Das nichtärztliche Personal im Rettungsdienst ................................................................. 9

1.3.5 Weichenstellung der Versorgung von Notfallpatienten ...................................................... 10

1.4 Der Notarzt und Rettungsdienst in Göttingen...............................................................11

1.5 Aktuelle Entwicklung der Notarzteinsätze im Rettungsdienst .........................................12

1.6 Potenzielle Nachteile einer niederschwelligen Alarmierung für den Notarzt..................14

1.7 Bisherige Bewertung der Notarzt-Indikation: der NACA-Score......................................15

1.8 Schema zur Beurteilung der Indikation von Notarzteinsätzen ..........................................16

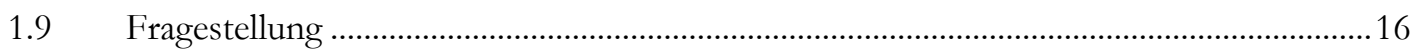

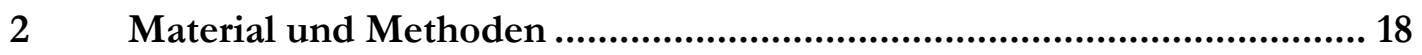

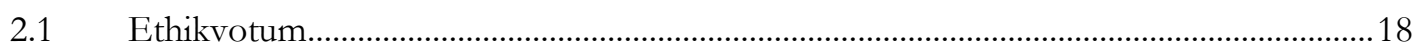

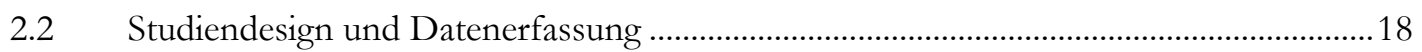

2.3 Kategorieneinteilung der erfassten Daten ...................................................................19

2.4 Kriterien der medizinischen Indikation für den Notarzteinsatz ......................................21

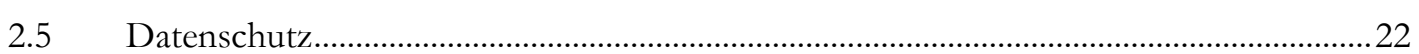

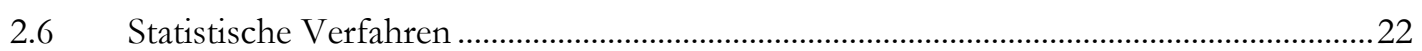

2.7 Literaturrecherche und Verwaltung der bibliographischen Quellen...............................22

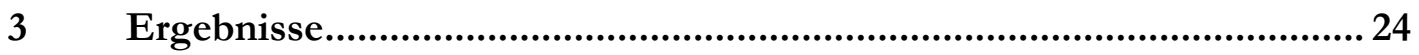

3.1 Verhältnis indiziert und nicht indizierter Notarzteinsätze ...................................................24

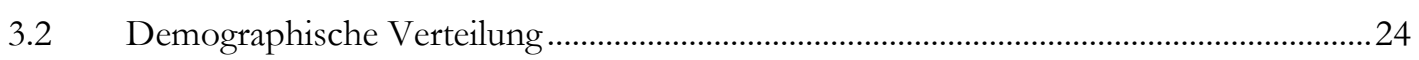

3.3 Die zwanzig häufigsten Alarmierungsmeldungen ..........................................................25

3.4 Die zehn Alarmierungsmeldungen mit dem höchsten Anteil einer gegebenen

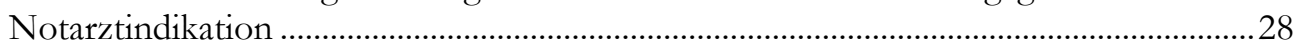

3.5 Die zehn Alarmierungsmeldungen mit dem niedrigsten Anteil einer gegebenen

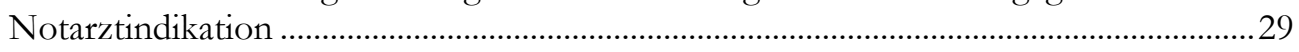

3.6 Anteil medizinisch indizierter Notarzteinsätze bei primärer Alarmierung vs. Nachalarmierung 
3.7 Anteil medizinisch indizierter Notarzteinsätze bei NEF-Einsätzen und RTH-Einsätzen

3.8 Anteil medizinisch indizierter Notarzteinsätze während und außerhalb regulärer Praxisöffnungszeiten

3.9 Anteil medizinisch indizierter Notarzteinsätze in den Gruppen von Notärzten mit und ohne Vorerfahrung im Rettungsdienst

3.10 Anteil medizinisch indizierter Notarzteinsätze bei traumatologischen und nicht traumatologischen Einsätzen.

3.11 Anteil medizinisch indizierter Notarzteinsätze in den Gruppen psychiatrische Notfälle und nicht psychiatrische Notfälle

3.12 Anteil medizinisch indizierter Notarzteinsätze in den Gruppen pädiatrische Notfälle und nicht pädiatrische Notfälle.

3.13 NACA-Score: Häufigkeitsverteilung und Korrelation mit dem Anteil medizinisch indizierter Notarzteinsätze

4 Diskussion.

4.1 Fragestellung, Grund für diese Untersuchung................................................................39

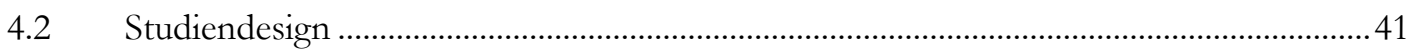

4.2.1 Bewertungssystem der Notarztindikationen ..................................................................41

4.2.2 Argumentation für eine prospektive Studie ................................................................43

4.2.3 Repräsentativität dieser Untersuchung ..............................................................................4 43

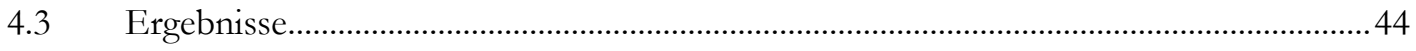

4.3.1 Verteilung der medizinischen Indikation der Notarzteinsätze......................................44

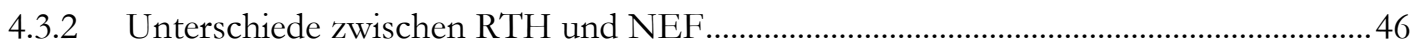

4.3.3 Verhältnisse bei primärer Alarmierung vs. Nachalarmierung ........................................47

4.3.4 Unterschiede nach Erreichbarkeit des Hausarztes............................................................49

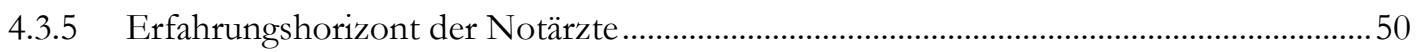

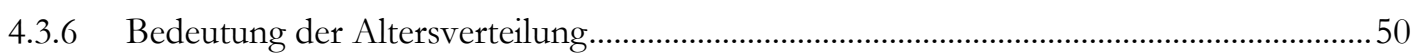

4.3.7 Besondere Alarmierungsmeldungen: Die Kindernotfälle, das Trauma, die

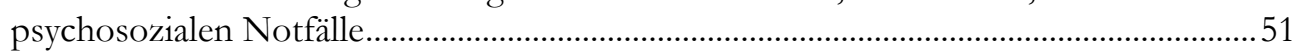

4.3.8 Der NACA-Score für die Bewertung der Notarzteinsätze...............................................54

4.4 Potenzielle Maßnahme zur selektiveren Alarmierung von Notärzten............................56

4.4.1 Verbesserung der Leistung der Rettungsleitstelle ..........................................................56

4.4.2 Berücksichtigung der aktuellen Entwicklungen im Rettungsdienst: Notfallsanitäter, verbesserte Ausstattung der Rettungsmittel, Telemedizin ..............................................58

4.5 Die unverzichtbare Rolle des Notarztes im Rettungsdienst........................................61

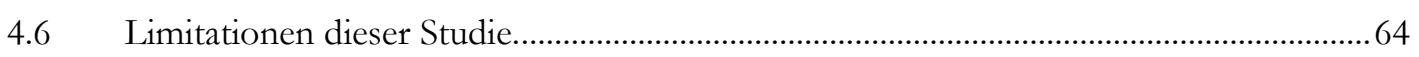

4.7 Schlussfolgerungen und Ausblicke für die Zukunft ...................................................65

$5 \quad$ Zusammenfassung.............................................................. 67

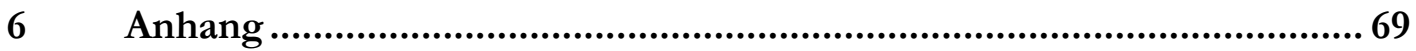

6.1 Fragebogen dieser Untersuchung für den Notarzt .....................................................69

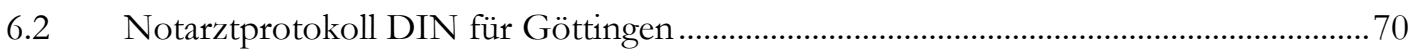

$7 \quad$ Literaturverzeichnis ..................................................................... 72 


\section{Abbildungsverzeichnis}

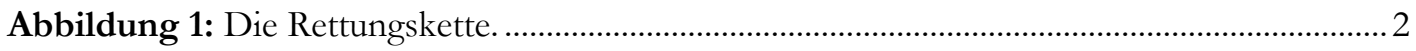

Abbildung 2: Ablauf eines Einsatzes im Rettungsdienst. ............................................................6

Abbildung 3: Entwicklung der Notarzteinsätze in der Stadt Heidelberg von 1984 bis 2004. .12

Abbildung 4: Verteilung der gesamten Notarzteinsätze hinsichtlich des Bestehens einer

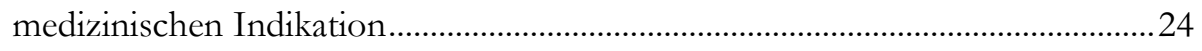

Abbildung 5: Altersverteilung der behandelten Patienten. ...........................................................25

Abbildung 6: Häufigkeitsverteilung der 20 häufigsten Alarmierungsmeldungen. ....................22

Abbildung 7: Häufigkeitsverteilung der zehn häufigsten Einsatzmeldungen...........................22

Abbildung 8: Zehn Alarmierungsmeldungen mit dem höchsten Anteil indizierter Notarztalarmierungen.

Abbildung 9: Zehn Einsatzmeldungen mit dem niedrigsten Anteil einer medizinischen Indikation.

Abbildung 10: Erstalarmierung vs. Nachalarmierung in Abhängigkeit von der medizinischen Indikation.

Abbildung 11: Graphik der Verteilung der medizinischen Indikation beim Einsatz des NEF vs. RTH.

Abbildung 12: Darstellung des Anteils der indizierten Einsätze vs. ohne medizinische Indikation nach Hausarzterreichbarkeit.

Abbildung 13: Anteil Notärzte ohne Vorerfahrung und mit Vorerfahrung im Ärzteteam in Göttingen

Abbildung 14: Verteilung der Einsätze mit und ohne medizinische Indikation nach Erfahrung der Notärzte.

Abbildung 15: Anteil der traumatologischen Notfälle an den Gesamteinsatzmeldungen ........34

Abbildung 16: Prozentuale Verteilung des Vorliegens einer medizinischen Indikation bei traumatologischen vs. nicht traumatologischen Notfällen....................................35

Abbildung 17: Anteil der psychiatrischen Notfälle an den Gesamteinsatzmeldungen..............35

Abbildung 18: Prozentuale Verteilung der medizinischen Indikation bei psychiatrischen Notfällen vs. anderer Einsatzmeldungen. .36

Abbildung 19: Prozentualer Anteil an medizinischer Indikation bei Kindernotfällen..............37

Abbildung 20: Korrelation des NACA-Scores und Einsätze mit medizinischer Indikation....38 


\section{Tabellenverzeichnis}

Tabelle 1: Indikationskatalog der BÄK für den Notarzteinsatz nach Beeinträchtigung der Vitalfunktionen

Tabelle 2: Indikationskatalog der BÄK für den Notarzteinsatz bezogen auf den Notfallhergang.

Tabelle 3: NACA-Score. .15

Tabelle 4: Angabe des Notarztes zu den Einsätzen................................................................19

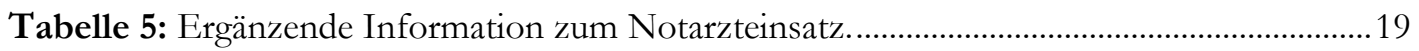

Tabelle 6: Kategorieneinteilung der erhobenen Daten................................................................220

Tabelle 7: Die zwanzig häufigsten Einsatzmeldungen und Angabe der jeweiligen medizinischen Indikation. 26

Tabelle 8: Verteilung der Einsätze nach NACA-Score... 38 


\section{Abkürzungsverzeichnis}

ACS (engl.) Acute coronary syndrom, Akutes Koronarsyndrom

ALS (engl.) Advanced life support, erweiterte Lebenserhaltungsmaßnahme

AP (1t.) Angina pectoris, Brustenge

BAND Bundesvereinigung der Arbeitsgemeinschaft der Notärzte Deutschlands

BÄK Bundesärztekammer

BAP bedingt ansprechbare Person

COPD chronische obstruktive Lungenerkrankung

CPR (engl.) Cardio pulmonary resuscitation, kardiopulmonale Wiederbelebung

DIN Deutsches Institut für Normung

EMS (engl.) Emergency Medical Services, Rettungsdienst

EMT (engl.) Emergency Medical Technician, Rettungssanitäter

EN Europäische Normen

ERC (engl.) European Resuscitation Council, Europäischer Wiederbelebungsrat

HRST Herzrhythmusstörung

KTW Krankentransportwagen

MU Motorradunfall

NA Notarzt

NÄ Notärzte

NACA (engl.) National Advisory Committee for Aeronautics, Nationales Beratungskommission für Luftfahrt

NAP nicht ansprechbare Person

NAW Notarztwagen

NEF Notarzteinsatzfahrzeug

OHEC (engl.) Out of Hospital Emergency Care, Notfallversorgung außerhalb des Krankenhauses

RA Rettungsassistent

$\mathrm{RD} \quad$ Rettungsdienst

ROSC (engl.) Return of spontaneous circulation, Rückkehr des spontanen Kreislaufs

RS Rettungssanitäter

RTH Rettungshubschrauber

RTW Rettungswagen

VU Verkehrsunfall 


\section{$1 \quad$ Einleitung}

\subsection{Die Rolle des Notarztes in der modernen präklinischen Notfallmedizin}

Der Notarzt stellt die höchst qualifizierte Instanz in der präklinischen Notfallmedizin. Sein Einsatz ist mit höheren Überlebensraten und weniger ausgeprägten Folgeerscheinungen nach einem Notfallereignis assoziiert (Hamilton et al. 2016). In der Notfallmedizin werden Patienten mit lebensbedrohlichen Erkrankungen oder Verletzungen durch qualifiziertes Personal so schnell wie möglich medizinisch versorgt, wobei nicht nur die Sicherung der Vitalfunktionen, sondern auch die Verhinderung weiterer Schäden und, wenn möglich, die Einleitung einer kausalen Therapie präklinisch im Vordergrund stehen (Wilson et al. 2015). Daran schließt sich der zügige Transport des Patienten in die nächstgelegene geeignete Klinik für die weitere innerklinische Behandlung an (Franschman et al. 2013).

Im Vergleich zu nicht arztbesetzen Systemen kommt ein breiteres Spektrum an Therapieverfahren zum Einsatz, wenn Notärzten bei der präklinischen Versorgung eingesetzt werden. Hieraus resultiert eine höhere Überlebensrate bei HerzKreislaufstillständen, respiratorischen Notfällen und erweiterter Traumaversorgung (Mikkelsen et al. 2015), sowie eine Steigerung der Kosteneffektivität der präklinischen Maßnahmen. Der Notarzt ermöglicht den Beginn einer intensivmedizischen Behandlung bereits am Notfallort, wobei zu beachten ist, dass im präklinischen Kontext die diagnostischen und therapeutischen Möglichkeiten, personelle Ressourcen und technische Verfahren in der Regel begrenzt sind (Roudsari et al. 2007).

Gemäß der Überlebenskette steht die schnellstmögliche Versorgung des Patienten im Vordergrund. Aus diesem Grund kommt das medizinische Personal zum Patienten an den Notfallort und nicht (wie lange praktiziert) umgekehrt. (Schulz-Stubner 2003). Der Notarzt und das nicht ärztliche medizinische Personal sind ein elementares Glied der Chain of Survival, im deutschen Rettungssystem spielt der Notfallmediziner deshalb eine entscheidende Rolle bei der Durchführung der frühzeitigen erweiterten lebensrettenden Maßnahmen (engl. Advanced Life Support, ALS) (Soar et al. 2015), gemäß den Leitlinien der European Resucitation Council (ERC - Europäischer Wiederbelebungsrat) und der erweiterten Traumaversorgung (engl. Advanced Trauma Life Support, ATLS) (Galvagno et al. 2019). Dies beinhaltet sowohl der Stellung korrekter Arbeitsdiagnosen bei seltenen oder komplexen Krankheitsbildern wie z. B. bei einer subarachnoidalen Blutung, als auch die Einleitung spezifischer Therapien, wie z. B. einer Thrombolyse bei einer schwerwiegenden Lungenarterienembolie (Everding et al. 2015). 


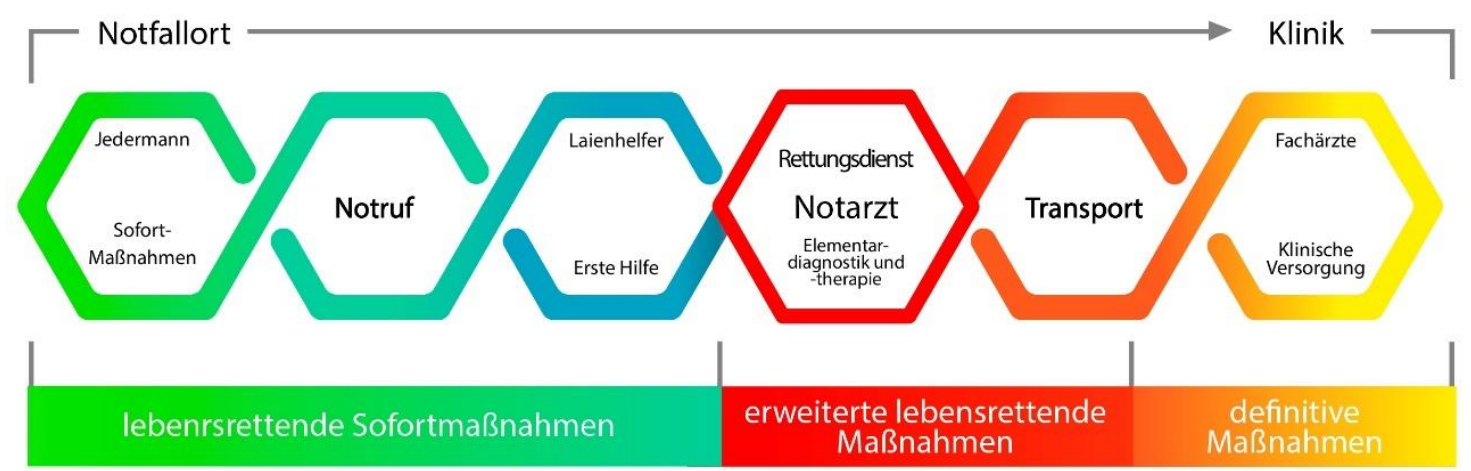

Abbildung 1: Die Rettungskette (nach Ahnefeld 2003) - (mit freundlicher Genehmigung von Georg Thieme Verlag KG).

Darüber hinaus entscheidet der Notarzt, welche Klinik für die weitere, spezifische Therapie des Patienten am besten geeignet ist. Studien konnten zeigen, dass die Zuteilung von Patienten an geeignete Einrichtungen zur Weiterbehandlung (fr. Triage) am besten durch Notärzte erfolgt (Marshall et al. 2017). Der Patient erreicht somit innerhalb kurzer Zeit das am besten geeignete Krankenhaus für die weitere Behandlung im Einsatzbereich. Darüber hinaus sorgt der Notarzt dafür, dass eine lückenlose Kommunikation mit den weiterbehandelnden Ärzten im Krankenhaus stattfindet (Harmsen et al. 2017), sodass präklinische Arbeitshypothesen und Verdachtsdiagnose gegebenenfalls übernommen und bereits eingeleitete Therapien nahtlos fortgeführt werden können. Diese medizinische Kompetenz ist nicht nur für Patienten im Herzstillstand oder Schock von Bedeutung, sondern auch für alle Patienten, die an einer Erkrankung leiden, bei der die golden hour, also die Zeit bis zur Einleitung einer kausalen Therapie, lebensentscheidend ist, wie z. B. bei einem Schlaganfall, einem akuten Myokardinfarkt, einer Sepsis oder einem Status Asthmaticus (Metelmann et al. 2018).

Der Notarzt qualifiziert sich in Deutschland durch die Zusatzbezeichnung Notfallmedizin für die Ausübung der präklinischen Notfallversorgung. Vertreten sind überwiegend die drei klinischen Fächer Anästhesie (Moecke und Knobelsdorff 2008), Chirurgie und Innere Medizin; jedoch auch in geringerer Verteilung die Pädiatrie und Neurologie. Die Notfallmedizin ist eine Zusatzbezeichnung, die bereits vor dem Abschluss der Facharztausbildung erworben werden kann.

\subsection{Historische Entwicklung des ärztlich betriebenen Rettungsdienstes}

Das heutige System zur präklinischen Notfallversorgung in Deutschland mit kurzen Hilfsfristen und rund um die Uhr verfügbaren Notärzten ist das Ergebnis einer langen Entwicklung. Einer der zentralen Gedanken war hierbei, den Patienten nicht wie bisher 
üblich schnellstmöglich vom Unfallort zu einem Arzt oder in eine Klinik zu transportieren, sondern den Arzt für die Akutversorgung zum Patienten zu bringen. Nachdem dieses Konzept mehr und mehr Fuß fasste, wurde das Einsatzspektrum der Notfallmedizin von der Versorgung von Patienten nach Unfällen oder Verletzungen auch auf andere schwere Erkrankungen mit akuter Lebensgefahr erweitert (Sefrin 2013).

Der Ursprung der Notfallmedizin geht auf das Militärwesen zurück, da kriegerische Auseinandersetzungen mit einer immer größeren Anzahl an schwer verletzten Soldaten einhergingen. Erst Ende des 19. Jahrhunderts wurde unter Mitbeteiligung des Internationalen Roten Kreuz ein erstes organisiertes Rettungssystem eingerichtet, das verletzte Personen auch außerhalb von Kriegshandlungen versorgte (Vanni et al. 2018).

Der erste Notarzt in heutigem Sinne war der Chirurg Marin Kirschner 1938 in Heidelberg, der mit seiner Forderung, den Arzt zum Patienten zu bringen, eines der zentralen Prinzipien des modernen deutschen Rettungsdienstes formulierte. Aus dieser Idee entstand ein fahrbarer Operationszug, der eine chirurgische Versorgung bereits unmittelbar am Unfallort ermöglichte. Diese Idee scheiterte jedoch an der eingeschränkten Mobilität des Systems (Dick 2006).

Ein weiterer Wegbereiter des Notarztdienstes war der Chirurg Karl Heinrich Bauer, der 1957 - ebenfalls in Heidelberg - ein Klinomobil implementierte. Dieses Einsatzfahrzeug bestand aus einem ein Omnibus mit Anhänger, in dem sich ein kompletter chirurgischer Arbeitsplatz befand, der gemäß dem Konzept von Kirschner samt Personal zum Unfallort gebracht werden sollte. Auch dieses Konzept stellte sich jedoch als überdimensioniert und so unflexibel heraus, so dass es sich nicht durchsetzen konnte.

Ebenfalls 1957 entwickelte Prof. Viktor Hoffmann in Köln ein flexibleres System für die präklinische Erstversorgung von Notfallpatienten. Auf der Basis eines umgebauten Lieferwagen (dem heutigen Notarztwagen entsprechend) wurde ein ärztlich besetztes Team nicht mit dem Ziel eines sofortigen operativen Eingriffes, sondern einer reinen Stabilisierung gestörter Vitalfunktionen bei lebensbedrohlichen Notfällen zum Einsatz gebracht (Burgkhardt und Burgkhardt 2007).

Um eine höhere Flexibilität bei der Entsendung des Notarztes zu erreichen, wurde 1964 mit einem Personenkraftwagen vom Typ VW-Käfer das erste Notarzteinsatzfahrzeug (NEF) etabliert und 1965 der Prototyp eines Rettungswagens (RTW) nach heutigem Standard (Deutsches Institut für Normen, DIN 75080) vorgestellt. Mit diesen Fahrzeugen war es möglich das von Prof. E. Gögler vorgestellte Rendezvous-System zu praktizieren (Sefrin 2003). Hierbei kann der Arzt sehr schnell mit Sondersignalen zum Einsatz gebracht werden und eine schnelle ärztliche Versorgung leisten, ohne zwangsläufig an den Transport des Patienten in die Klinik gebunden zu sein. Nach dem Vorbild dieses Projektes nahmen zahlreiche Abteilungen für Chirurgie und besonders Anästhesiologie in den darauffolgenden Jahren arztbesetzten Einsatzfahrzeuge in Betrieb, wie z. B. in Köln, München und Mainz. 
In den 1950er Jahren gewannen die frühestmögliche Schockbehandlung, Reanimation mit Intubation und künstliche Beatmung sowie Herzdruckmassage und Defibrillation zunehmend an Bedeutung (Ahnefeld 2003), wodurch die therapeutische Lücke zwischen Notfallort und der innerklinischen Endversorgung geschlossen werden sollte. Professor Friedrich W. Ahnefeld formulierte 1966 das Konzept der Rettungskette für die stufenweise Versorgung von Notfallpatienten von der Alarmierung bis zum Eintreffen in die Notaufnahme (Dick 2002). Professor R. Frey, der erste Ordinarius für Anästhesiologie in der damaligen Bundesrepublik Deutschland, förderte sehr stark die Weiterentwicklung der präklinischen Notfallmedizin. Die rasche und intensive Weiterentwicklung notarztbesetzer Rettungssysteme wurde auch durch kontinuierliche Fortschritte der Medizintechnik ermöglicht. Einer der bedeutendsten Meilensteine war die offizielle Indienstnahme des ersten Rettungshubschrauber „Christoph 01“ in München im Jahre 1970 (Klingshirn 2001). Auch das Einsatzspektrum dieses initial für die Traumaversorgung gedachten Notarztsystems wurde im Verlauf der Zeit mehr und mehr auf andere, nicht traumatologische Notfälle erweitert.

Mitte der siebziger Jahre wurde das Rettungssystem in der Bundesrepublik Deutschland flächendeckend in allen Bundesländern auf der Grundlage eines von Bund und Ländern gemeinsam erstellten Musters für Landesrettungsdienstgesetze ausgebaut. Nach Anregung der Deutschen Gesellschaft für Anästhesie und Intensivmedizin (DGAI) und der fachübergreifenden Deutschen Interdisziplinären Vereinigung für Intensiv- und Notfallmedizin (DIVI) wurde eine Empfehlung für die Qualifikation des Notärztlichen Personals formuliert. Die Bundesärztekammer definierte Zugangsvoraussetzungen für den Erwerb des Fachkundenachweis Rettungsdienst, der für die Teilnahme am Notarztdienst gefordert wurde und 2003 durch die „Zusatzbezeichnung Notfallmedizin“ abgelöst wurde.

In der ehemaligen Deutschen Demokratischen Republik (DDR) erfolgte ebenfalls die Entwicklung eines arztbesetzten Rettungssystems. Bereits 1960 wurde in Magdeburg ein mit Anästhesisten besetztes Einsatzfahrzeug eingerichtet. Dieses Fahrzeug wurde unter dem Namen Schnelle Medizinische Hilfe (SMH) in Zusammenarbeit mit der Feuerwehr ursprünglich zur Versorgung von Unfallverletzten in Dienst gestellt (Ebmeyer und Röse 2013). Bald stellte sich heraus, dass nicht nur traumatisierte, sondern auch internistische Patienten bei vital bedrohlichen Zuständen von einer notärztlichen Erstversorgung profitieren können, so dass das Rettungsdienstsystems in Dringliche Medizinische Hilfe (DMH) umbenannt wurde. Durch einen kontinuierlichen Ausbau konnten nahezu 80\% der Bevölkerung innerhalb angemessener Zeit erreicht werden. Ein Lufttransport war in der DDR nicht vorgesehen und fand nur selten unter Mitwirkung sowjetischer Streitkräfte statt (Jantzen et al. 2008). Mit der Wiedervereinigung wurde der Rettungsdienst in den neuen Bundesländern von den ehemals westdeutschen Hilfsorganisationen übernommen und an einheitlichen Standards ausgerichtet. 
Die Klinik für Anästhesiologie der Universitätsmedizin Göttingen (ehemals Zentrum für Anästhesiologie, Rettungs- und Intensivmedizin) trug mit der Etablierung des ersten Notarztwagens in Niedersachsen im Jahre 1971 und der Stationierung des Rettungshubschraubers Christoph 44 im Jahre 1980 maßgeblich zur Entwicklung der präklinischen Notfallmedizin bei (Roessler 2019a).

\subsection{Organisation des Rettungsdienstes}

Die Versorgung von Verletzten oder Erkrankten außerhalb der Klinik (engl. OHEC, Out of Hospital Emergency Care) findet in den einzelnen Ländern in Abhängigkeit von deren sozioökonomischem Entwicklungsgrad auf sehr unterschiedlichen medizinischen Niveaus statt. Sie reicht vom schnellen, unorganisierten Abtransportes durch Sicherheitskräfte oder Privatpersonen über mehr oder weniger geeignete Erste-Hilfe-Stellen bis zu hin zu organisierten Rettungssystemen mit professionellen medizinischen Fachkräften (Suryanto et al. 2017). In der Europäischen Union, den USA und der ehemaligen „Commonwealth“ verfügen alle Länder über moderne präklinische notfallmedizinische Versorgungssysteme (engl. Emergency Medical Service, EMS). Obwohl einige gemeinsame Leitlinien und Prinzipien existieren, wie zum Beispiel die einheitliche Notrufnummer (112 innerhalb Europas), gibt es jedoch keine einheitlichen Standards für die Organisation des Rettungsdienstes (Fischer et al. 2011).

Sämtliche Systeme der präklinischen Versorgung lassen sich in zwei Gruppen einteilen: Das anglo-amerikanische System, in dem die Erstversorgung und lebensrettende Maßnahmen durch ausgebildetes nicht ärztliches Personal (engl. Paramedic und engl. Emergency Medical Technician, EMT) geleistet wird, steht dem deutsch-französischen System gegenüber, welches durch die Verfügbarkeit von spezialisierten Notfallmedizinern (Al-Shaqsi 2010) gekennzeichnet ist. Letzteres gilt als maximaler Standard der Versorgungsqualität in der präklinischen Medizin, so dass in den letzten Jahren tendenziell ein Wandel hin zu Rettungsdiensten mit ärztlichem Personal, insbesondere für den Einsatz von Rettungshubschraubern, stattgefunden hat.

Die Bundesrepublik Deutschland verfügt heutzutage über ein lückenloses Netz von Rettungswachen und bodengebundenen sowie luftgestützten Notarztsystemen. Eine definierte Hilfsfrist von 10 - 15 Minuten vom Eingang des Notrufes bis zum Erreichen des Notfallorts kann in 95\% der Fälle eingehalten werden (Lechleuthner 2019). Dies erfordert eine ausreichend hohe Anzahl verfügbarer Fahrzeuge und eine optimale strategischräumliche Stationierung. Das deutsche Rettungsdienstsystem zählt aufgrund seiner Leistungsmerkmale zu den besten der Welt (Roessler und Zuzan 2006). Der Rettungsdienst wird aufgrund des Föderalismusprinzips im deutschen Grundgesetz auf Länderebene organisiert, dennoch existieren geringe Unterschiede in der Organisation des Rettungsdienstes zwischen den Bundesländern. Die Verantwortung für die Organisation und Durchführung des Rettungsdienstes wird an die Kreise bzw. kreisfreien Städte als Träger des 
Rettungsdienstes weitergegeben. In dieser Arbeit liegt der Fokus auf dem Land Niedersachsen, und besonders auf der Stadt Göttingen und Umgebung.

Das System ist dual gestaltet und verfügt neben nichtärztlichem Personal auch über speziell qualifizierte Notfallmediziner, die in lebensbedrohlichen Fällen eingesetzt werden. Ziel ist die Aufrechterhaltung der Rettungskette von der Notfallstelle bis zum Ort der endgültigen Versorgung (Stevanovic et al. 2017).

Jedem Bürger steht in der Bundesrepublik das Recht auf eine qualifizierte Hilfeleistung zu jeder Zeit zu. Falls notwendig, soll diese Leistung durch einen Notfallmediziner erbracht werden. Laut Statistiken der Bundesanstalt für Straßenwesen wird in etwa 40\% der Notfallsituationen ein Notarzt hinzugezogen (Schmiedel und Behrendt 2015).

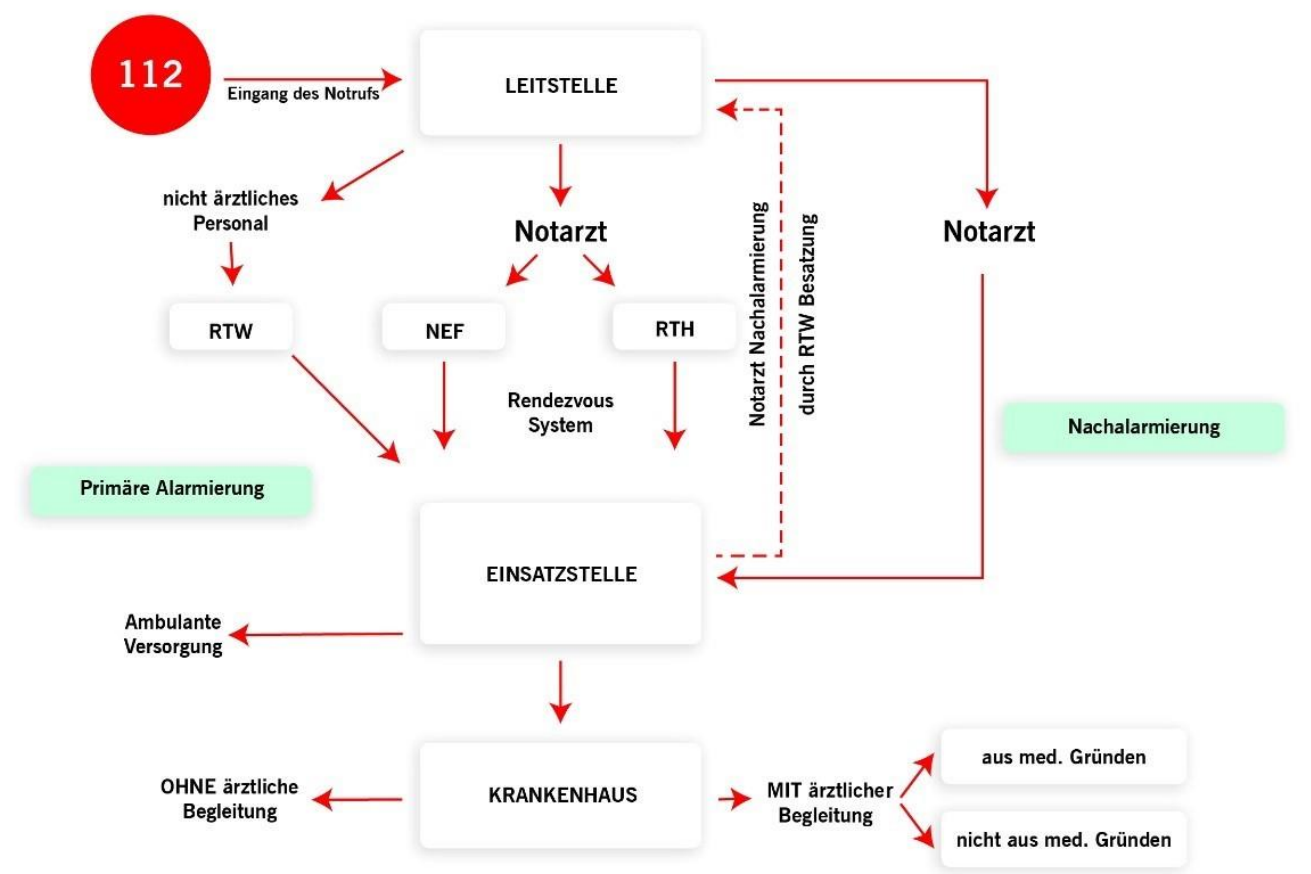

Abbildung 2: Ablauf eines Einsatzes im Rettungsdienst

\subsubsection{Die Rettungsleitstelle}

Alle Fahrzeuge des Rettungsdienstes werden von einer Leitstelle alarmiert und koordiniert. Neben reinen Rettungsleitstellen setzen sich mehr und mehr integrierte Leitstellen durch, in denen auch Einätze der Feuerwehr oder der Polizei organisiert und gesteuert werden. Notrufe werden in der Leitstelle durch geschultes Personal entgegengenommen. Leitstellendisponenten verfügen über mindestens eine Rettungsassistentenausbildung und eine spezielle Zusatzausbildung Leitstelle sowie über Detailkenntnisse der regionalen 
Infrastruktur. Die Leitstelle muss für Notfallmeldungen ständig erreichbar sein. Die Leitstelle verfügt über das Weisungsrecht für die Einsatzkräfte, jedoch nicht in Bezug auf medizinische und flugtechnische Angelegenheiten (Maurer et al. 2015).

Abhängig von der am Telefon beschriebenen Lage vor Ort wird entschieden, ob primär nur einen Rettungswagen zum Notfallort entsendet wird, oder zusätzlich ein arztbesetztes Rettungsmittel, wenn der Leitstellendisponent von einer Notfallsituation mit akuter Lebensgefahr bzw. erheblicher Schädigung des Patienten ausgeht (Kumpch und Luiz 2011). Die Entscheidung über die Aussendung des Notarztes zur Notfallstelle stellt eine der wichtigsten Aufgaben der Leitstelle dar. Als Grundlage für diese Entscheidung existiert in Deutschland ein Indikationskatalog mit Einsatz- und Zustandsstichwörtern, in welchen Situationen ein Notarzt hinzugezogen werden sollte.

\subsubsection{Die Einsatzmittel im Rettungsdienst}

Aktuell existieren in Deutschland circa 1800 Rettungswachen, an denen etwa 3400 Rettungswagen und ca. 1000 arztbesetzte Fahrzeuge stationiert sind. Das Luftrettungssystem verfügt von 53 Hubschrauberstationen mit einem Einsatzradius von 50 - 70 Kilometern. Der bodengebundene Rettungsdienst steht unter Verantwortung der lokalen Behörden, für das Hubschraubersystem haben die Länder direkte Zuständigkeit (Fischer et al. 2003).

Die bodengebundenen Einsatzmittel des Rettungsdienstes werden im Folgenden erläutert:

Die niedrigste Instanz der bodengebundenen Einsatzfahrzeuge ist der Krankentransportwagen (KTW). Der KTW muss mindestens zwei Personen als Besatzung haben, typischerweise zwei Rettungssanitäter, oder einen Rettungsassistenten und einen Rettungssanitäter. Da der KTW nicht für eine differenzierte Behandlung von akuten Notfällen ausgestattet ist, wird dieser nur für den Transport von Patienten ohne vitale Gefährdung eingesetzt, z. B. für Dialysefahrten oder elektive Krankenhauseinweisungen.

Die nächsthöhere Instanz bodengebundener Rettungsmittel ist der Rettungswagen (RTW), der für die Versorgung und den Transport von Notfallpatienten konzipiert wurde. Seine standardisierte medizinisch-technische Ausstattung (DIN EN 1789 Typ C) erlaubt die Versorgung, Überwachung und den Transport von Notfallpatienten. Seit der Einführung des Notfallsanitätergesetzes in Niedersachsen ist es vorgeschrieben, dass mindestens ein Notfallsanitäter zur Besatzung des Rettungswagens gehören muss. Vor Inkrafttreten dieses Gesetzes bestand die Besatzung von RTW aus zwei Rettungsassistenten oder einem Rettungsassistenten und einem Rettungssanitäter.

Das am weitesten verbreitete arztbesetzte Rettungsmittel ist das Notarzteinsatzfahrzeug (NEF), das einen schnellen und flexiblen Transport des Notarztes an die Einsatzstelle ermöglicht. Das Fahrzeug verfügt über eine umfangreiche medizinische Ausstattung für die Behandlung von lebensbedrohlichen Zuständen, es hat jedoch weder einen Behandlungsraum noch Transportmöglichkeiten für Patienten. Das NEF ist für den Einsatz 
im „Rendezvous-System“ (siehe unten) konzipiert. Die nichtärztliche Besatzung des Notarzteinsatzfahrzeuges (der NEF-Fahrer) muss mindestens als Rettungsassistent qualifiziert sein.

Ein früher sehr verbreitetes, heute aber kaum noch eingesetztes arztbesetztes Rettungsmittel ist der Notarztwagen (NAW), der ebenfalls über die vollständige medizinisch-technische Ausstattung zur Versorgung, Überwachung und Beförderung von Notfallpatienten verfügt. Die Besatzung des NAW besteht aus einem Arzt und zwei nicht ärztlichen Besatzungsmitgliedern, die als Rettungsassistent oder Notfallsanitäter qualifiziert sein müssen. Aufgrund seiner Inflexibilität (der Notarzt ist fest an das Fahrzeug gebunden) wurden die NAW in Deutschland nahezu vollständig durch NEF ersetzt.

Ebenfalls dem Rettungsdienst zuzuordnen sind Intensivtransportwagen (ITW), die über eine besondere Ausstattung für den Interhospitaltransfer von intensiv überwachungs- und behandlungspflichtigen Patienten verfügen. Diese besteht in speziellen Beatmungsgeräten, Medikamenten, Motorspritzenpumpen und deutlich erweitertem Monitoring. Neben zwei Rettungsassistenten bzw. Notfallsanitätern sind ITW mit Ärzten besetzt, die über eine spezielle Ausbildung im Interhospitaltransfer von Intensivpatienten verfügen. Auch wenn die ITW formal dem Rettungsdienst zugeschrieben werden übernehmen ITW nur selten Primäreinsätze, da ihr Einsatz in der Regel eine Vorlaufzeit benötigt, die weit über den maximalen Hilfsfristen liegt.

Im Gegensatz zu den bodengebundenen Rettungsmitteln nehmen Luftrettungsmittel eine Sonderstellung ein: Ihr Einsatz ist nur bei Tageslicht und ausreichenden Sichtverhältnissen möglich, so dass ihre Einsatzbereitschaft nachts grundsätzlich nicht gegeben ist, und auch tagsüber stets einer gewissen Unsicherheit unterliegt. Aus diesem Grunde stellen sie eine subsidiäre Ergänzung zum bodengebundenen Rettungsdienst dar.

Medizinisch gelten die gleichen Kriterien für den Einsatz des Hubschrauber-Notarztes wie für den Einsatz des NEF (Indikationskatalog), die Entscheidung ob ein NEF oder ein Rettungshubschrauber alarmiert wird, ist rein einsatztaktischer Natur. (Gries et al. 2008). Die Zeitverkürzung bis zum Eintreffen am Einsatzort sowie für den Transportweg kann bei kritischen Zuständen, insbesondere für Trauma Patienten, die Entscheidung über die Entsendung eines Luftrettungsmittels beeinflussen (Stewart et al. 2019 und Wigman et al. 2011).

Rettungstransporthubschrauber (RTH) sind spezielle Hubschrauber, die über eine vergleichbare medizinische Ausrüstung verfügen wie ein NEF. RTH werden einerseits als schneller und flexibler Notarztzubringer im Rahmen des Rendezvous-Systems eingesetzt. Im Gegensatz zum NEF können in RTH jedoch auch Patienten vom Einsatzort in die Zielklinik transportiert werden. Dies ist insbesondere dann von Vorteil, wenn eine schnellstmögliche klinische Versorgung erforderlich ist, wie z. B. bei intraabdominellen Blutungen. Für zeitkritische Verlegungen zwischen Krankenhäusern ("Sekundäreinsätze") werden ebenfalls oft RTH angefordert, wenn die Vorlaufzeit von reinen Verlegungs-Hubschraubern (s.u.) zu 
inakzeptablen Zeitverlusten führen würde. Die Möglichkeiten zur Behandlung des Patienten während des Fluges sind aufgrund der eingeschränkten Räumlichen Verhältnisse sehr begrenzt. Die Besatzung eines RTH besteht aus einem Piloten, dem Notarzt sowie einem „HMC“ (engl. Helicopter Emergency Medical Services Crew Member), d. h. einem Rettungsassistenten/Notfallsanitäter mit einer Zusatzausbildung in Flugtechnischen Aspekten wie z. B. Navigation.

Intensivtransporthubschrauber (ITH) zählen ebenfalls zu den Luftrettungsmitteln, ihre primäre Aufgabe ist der Interhospitaltransfer von intensivpflichtigen Patienten. Analog zum ITW verfügen ITH ebenfalls über eine erweiterte intensivmedizinische Ausrüstung, der Versorgungs- und Behandlungsraum ist deutlich größer als im RTH. Bedingt durch die meist längere Vorlaufzeit bis zur Einsatzbereitschaft und ihre Größe (die wiederum die Auswahl an Landemöglichkeiten einschränkt) werden Intensivtransporthubschrauber nur in Ausnahmefällen für Primäreinsätze alarmiert.

\subsubsection{Das Rendezvous-System}

Der Grundgedanke des Rendezvous-Systems (fr. Termin) besteht darin, dass der Notarzt parallel zum Rettungswagen alarmiert wird, jedoch unabhängig davon mit einem Notarzteinsatzfahrzeug oder einem Rettungshubschrauber zum Einsatzort gebracht wird. Dieses System erfordert zwar mindestens zwei Fahrzeuge, daher ist es funktionell aufwendiger. Das Rendezvous-System ermöglicht jedoch kürzere Eintreffzeiten sowie flexiblere Einsatzmöglichkeiten für den Notarzt: Nach der Erstversorgung muss der Notarzt den Transport ins Krankenhaus nur dann begleiten, wenn die medizinische Situation dies erfordert. Andernfalls ist der Notarzt nach der Erstversorgung wieder einsatzbereit und steht der Leitstelle sofort wieder zur Verfügung (Hecker und Domres 2018). Aktuell wird in Deutschland bei Notarzteinsätzen fast ausschließlich das Rendezvous-System praktiziert.

\subsubsection{Das nichtärztliche Personal im Rettungsdienst}

Laut Niedersächsischem Rettungsdienstgesetz (NRettDG) muss das im Rettungsdienst eingesetzte Personal "geeignet sein und die erforderliche Zuverlässigkeit besitzen " ( $\int 10$ NrettDG). Das Gesetz gibt Empfehlungen für Mindeststandards vor, derzeit existieren vier Qualifikationseben für das nichtärztliche Personal im Rettungsdienst (Lippert 2013):

Die unterste Qualifikationsstufe ist der Rettungshelfer (RH). Die Ausbildung zum Rettungshelfer umfasst 240 Stunden, aufgeteilt in 160 Stunden Theorie und 80 Stunden praktische Ausbildung mit Fokus auf Wiederbelebung und Notfallversorgung. Rettungshelfer arbeiten meistens als Freiwillige bei öffentlichen Veranstaltungen und bei der nicht dringlichen Patientenbeförderung.

Die nächst höhere Qualifikationsebene ist der Rettungssanitäter (RS). Die Ausbildung zum Rettungssanitäter umfasst 520 Stunden, davon 160 Stunden Theorie, 160 Stunden Praktikum im Krankenhaus, 160 Stunden Praktikum auf einer Rettungswache sowie ein 40- stündiger 
Abschlusslehrgang. Bis 1989 war der Rettungssanitäter die am häufigsten eingesetzte Berufsgruppe auf Rettungsmitteln. Mit Inkrafttreten des Rettungsassistentengesetz wurden Rettungssanitäter nach und nach durch Rettungsassistenten abgelöst.

Der Rettungsassistent (RA) verfügt im Gegensatz zu den zuvor genannten Berufsgruppen über eine geschützte Berufsbezeichnung. Die Ausbildung dauert zwei Jahre und beinhaltet umfangreiche theoretische Lerninhalte sowie eine praktische Ausbildung über 1600 Stunden im Rettungsdienst.

Am Konzept des Rettungsassistenten wurde kritisiert, dass die zweijährige Ausbildung im Vergleich zu anderen dreijährigen Lehrberufen von nichtärztlichem Personal im Gesundheitssystem (z. B. bei Gesundheits- und Krankenpfleger, Hebammen usw.) deutlich kürzer ist und den zunehmend komplexeren Anforderungen und Ansprüchen des modernen Rettungsdienstsystems und der Entwicklung in der Notfallmedizin nicht mehr gerecht wird (Brokmann et al. 2013). Seit 2014 wurde daher das neue Berufsbild des „Notfallsanitäters“ eingeführt (Flake et al. 2013), für dessen Erwerb eine dreijährige Ausbildung zu absolvieren ist, die mit einer staatlichen Prüfung abschließt. Für diese ebenfalls geschützte Berufsbezeichnung werden zunehmend Auszubildende zugelassen. Für den Rettungsdienst ist die Tatsache relevant, dass Notfallsanitätern die Durchführung bestimmter ärztlicher Maßnahmen unter Anwendung von Algorithmen als Regelkompetenz zugesprochen wird. Diese Maßnahmen sind jedoch nicht vereinheitlicht, sondern von den ärztlichen Leitern der Rettungsdienste zu definieren.

Rettungshelfern, Rettungssanitätern und Rettungsassistenten ist es dagegen formell nicht erlaubt, ärztliche Maßnahmen selbständig durchzuführen (Ahnefeld et al. 2003). Ist im Falle einer akut lebensbedrohlichen Situation jedoch kein Notarzt verfügbar, ist die Durchführung von lebensrettenden Maßnahmen wie die Wiederbelebung, Defibrillation, die Gabe von Glukose bei einer Hypoglykämie oder Adrenalin bei einer starken anaphylaktischen Reaktion unter der Bezeichnung „Notkompetenz“ (Ohr 2005) durch dieses Personal möglich (Timmermann et al. 2008). In Anwesenheit eines Notarztes ist das nichtärztliche Personal dem Arzt untergeordnet.

Trifft nichtärztliches Personal als erstes an der Einsatzstelle ein, kann es nach Einschätzung der Lage jederzeit über die Leitstelle einen Notarzt nachfordern, wenn der Zustand des Patienten die Notwendigkeit ärztlicher Interventionen erkennen lässt (z. B. bei einem hämodynamisch instabilen Herzinfarkt), oder wenn medikolegale Aspekte dies erfordern (z. B. bei Transportverweigerung des Patienten trotz gegebener Behandlungsindikation).

\subsubsection{Weichenstellung der Versorgung von Notfallpatienten}

Wenn die Erstversorgung des Patienten am Einsatzort abgeschlossen ist, kann der Patient entweder vor Ort (i. d. R. zu Hause) belassen werden, oder aber zur weiteren Behandlung in das nächstgelegene geeignete Krankenhaus transportiert werden. 
Für Krankenhäuser in Deutschland sind je nach Komplexität und Spezialisierungsgrad drei medizinische Versorgungstufen definiert (Bayer et al. 2013). Die erste Stufe ist die Grundund Regelversorgung, diese Krankenhäuser verfügen über Abteilungen für Innere Medizin und Allgemeinchirurgie. Die zweite Versorgungsstufe ist die Schwerpunktversorgung, die zusätzlich zur Grund- und Regelversorgung über zusätzliche Fachrichtungen wie Kardiologie, Nephrologie Gastroenterologie, Neurologie, Geburtshilfe, Orthopädie/Unfallchirurgie verfügt. Die dritte Stufe stellt die Maximalversorgung dar; diese beinhaltet alle Versorgungsdisziplinen, eine hohe fachliche Spezialisierung, eine zentrale Notaufnahme und einen Schockraum.

Patienten mit komplexen Erkrankungen oder Zuständen, die eine spezialisierte Behandlung erfordern - z. B. Polytraumata, Kindererkrankungen, ein akuter Myokardinfarkt (Dick 2003), Intrakranielle Blutung (Doggen et al. 2016) - profitieren von der Behandlung in speziellen Zentren. Daher muss nicht immer zwangsläufig das nächstgelegenen Krankenhaus angefahren, sondern das am besten für die weitere Versorgung geeignete (Strohm et al. 2018). Nach Beendigung des Einsatzes erfolgt die Rückmeldung an die Leitstelle, mit der das Rettungsmittel als frei und einsatzbereit gemeldet wird.

\subsection{Der Notarzt und Rettungsdienst in Göttingen}

Für die Stadt Göttingen und das angrenzende Umland werden die Notärzte aus der Klinik für Anästhesiologie der Universitätsmedizin Göttingen bereitgestellt. Dies geschieht in einer Kooperation mit der Berufsfeuerwehr Göttingen (diese betreibt die Notarzteinsatzfahrzeuge) und der Deutschen Rettungsflugwacht.

Im Bundesland Niedersachsen werden als Notarzt nur approbierte Ärzte eingesetzt, die die Qualifikation „Zusatzbezeichnung Notfallmedizin“ erworben haben. Voraussetzungen für den Erwerb dieser Zusatzbezeichnung sind (gemäß \6 Abs. 1 der Weiterbildungsordnung) eine 24-monatige Weiterbildung in einem Fachgebiet der stationären Patientenversorgung, sechs Monate Weiterbildungszeit in der Intensivmedizin, Anästhesiologie oder in der Notfallaufnahme, die Teilnahme an einem 80 Stunden-Kurs in allgemeiner und spezieller Notfallbehandlung sowie 50 Einsätze im Notarztwagen oder Rettungshubschrauber unter Anleitung eines verantwortlichen Notarztes bzw. 20 Einsätze im Notarztwagen oder Rettungshubschrauber unter Anleitung eines weiterbildungsermächtigten Notarztes. Abschließend muss eine Prüfung in Form eines kollegialen Gespräches bei der Ärztekammer abgelegt werden.

Die Stadt Göttingen verfügt über drei arztbesetzte Rettungsmittel, mit denen rund um die Uhr zwei Notärzte für Einsätze im Rendezvous-System zur Verfügung stehen: An beiden Feuerwachen der Stadt ist jeweils ein NEF stationiert (Feuerwache Süd: NEF 1-82-1, Feuerwache Nord: 2-82-1), der Rettungshubschrauber Christoph 44 der DRF-Luftrettung ist am Universitätsklinikum stationiert. 
Die Notärzte absolvieren 24 stündige Dienste, von 07:00 Uhr bis 07:00 Uhr des nächsten Tages. Das NEF 2-82-1 ist 24 Stunden im Dienst, während das NEF 1-82-1 komplementär zum RTH Christoph 44 eingesetzt wird, d. h. es wird erst nach Sonnenuntergang in Betrieb genommen und mit dem Notarzt des RTH besetzt.

Während im Jahr 2015 in Göttingen etwa 3600 Notarzteinsätze stattfanden, werden Notärzte aktuell zu etwa 5000 Einsätzen pro Jahr alarmiert (Roessler 2019b). Hiervon werden etwa 2000 bis 2800 Patienten durch das NEF-Nord versorgt, 500 bis 900 Patienten durch das NEF-Süd, und ca. 1000 bis 1500 Patienten durch den RTH Cristoph 44.

\subsection{Aktuelle Entwicklung der Notarzteinsätze im Rettungsdienst}

Die Häufigkeit von Notarzteinsätzen hat in den letzten Jahrzehnten in erheblichem Umfang zugenommen. Ebenso ist eine Zunahme von Patienten zu verzeichnen, die eine Notaufnahme über die Inanspruchnahme des Rettungsdienstes aufsuchen (Osterloh 2019). Für den Zeitraum zwischen 1984 und 2004 wird eine Vervierfachung der Notarzteinsätze in Heidelberg (957 Einsätze in 1984 vs. 3825 Einsätze in 2004) beschrieben (Bernhard et al. 2006), die als repräsentativ für das gesamte Bundesgebiet angesehen werden kann. Für die Stadt Leipzig beschreiben Bader et al. eine Zunahme der Notarzteinsätze um 24\% im Zeitraum 2003 bis 2013 (6030 Einsätze in 2003 vs. 7470 Einsätze in 2013) (Bader et al. 2018), die nicht auf das Bevölkerungswachstum zurückzuführen sind (Anstieg der Einsätze pro 1000 Einwohner).

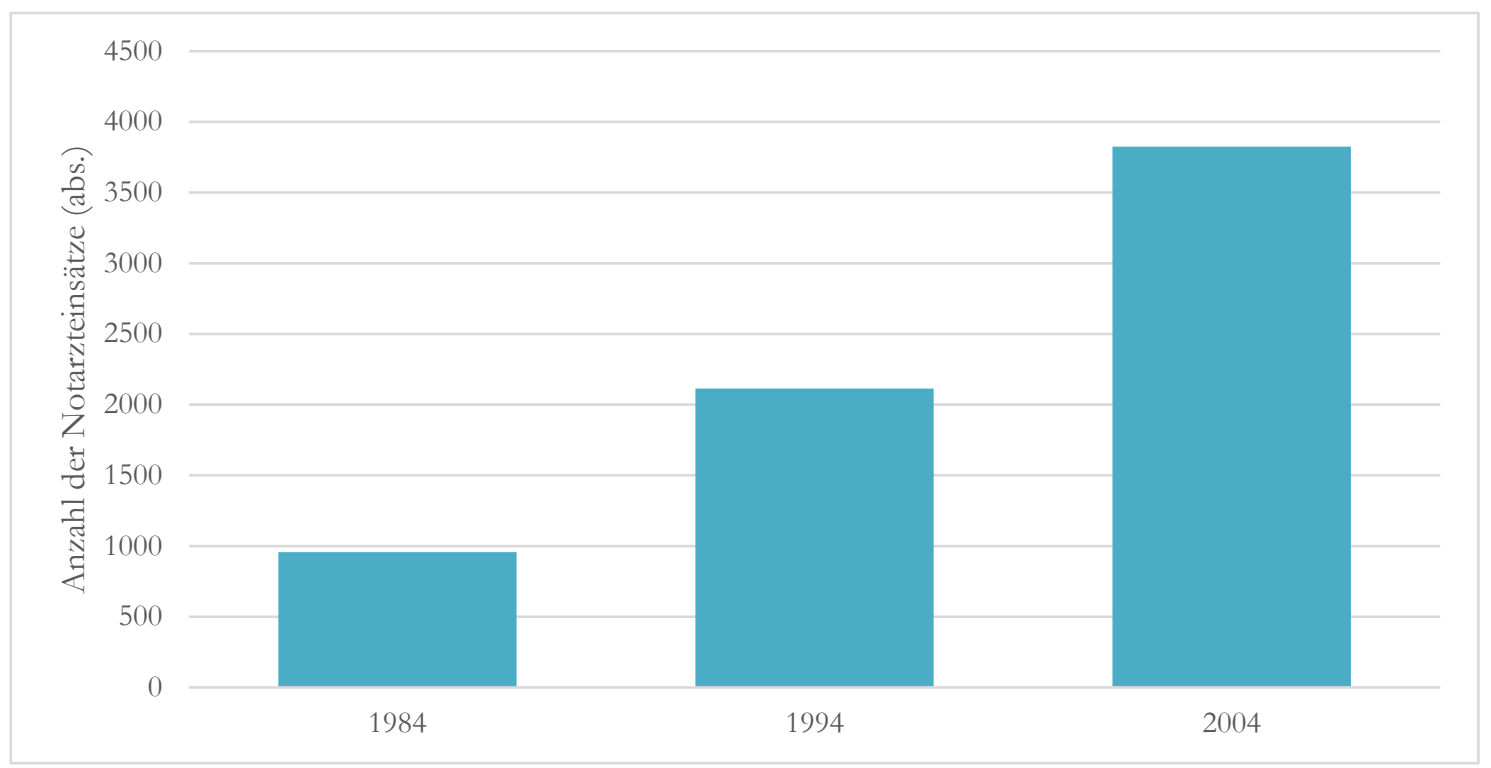

Abbildung 3: Entwicklung der Notarzteinsätze in der Stadt Heidelberg von 1984 bis 2004 (nach Bernhard et al. 2006). 
Da nicht anzunehmen ist, dass die Inzidenz von vital bedrohlichen Erkrankungen oder Unfällen mit schweren Personenschäden ebenfalls in diesem Maße gestiegen ist, könnte ein möglicher Grund für diese massive Zunahme an Notarzteinsätzen eine zunehmend niederschwellige Alarmierungsstrategie arztbesetzter Rettungsmittel anhand des Indikationskatalogs für die Rettungsleitstellen sein (BÄK 2013). Der Notarztindikationskatalog listet sowohl Unfallhergänge als auch medizinisch-klinische Zustände auf, die eine relevante Beeinträchtigung von Vitalfunktionen vermuten lassen. Anhand dieser Alarmierungsliste wird der Notarzt bei Verdacht einer potenziellen Lebensgefahr zur Einsatzstelle entsendet. In der Tabelle 1 und Tabelle 2 werden diese Alarmierungsmeldungen aufgeführt. Bei der Zusammenstellung der NotarztIndikationskataloge stand eine möglichst hohe Sensitivität für die Erfassung akut lebensbedrohlicher Zustände im Vordergrund, die mit einer entsprechend reduzierten Spezifität einhergeht.

Diese Einsatzstrategie einer eher großzügigen Alarmierung arztbesetzter Rettungsmittel wurde bislang kaum in Frage gestellt.

Tabelle 1: Indikationskatalog der BÄK für den Notarzteinsatz nach Beeinträchtigung der Vitalfunktionen (nach BÄK 2013).

\begin{tabular}{|c|c|c|}
\hline Funktionen & Zustand & Beispiel \\
\hline Bewusstsein & $\begin{array}{l}\text { reagiert nicht oder nicht adäquat auf } \\
\text { Ansprechen und Rütteln }\end{array}$ & $\begin{array}{l}\text { Schädel-Hirn-Trauma (SHT), } \\
\text { Schlaganfall, Vergiftungen, } \\
\text { Krampfanfall, Koma }\end{array}$ \\
\hline Atmung & $\begin{array}{l}\text { keine normale Atmung, ausgeprägte oder } \\
\text { zunehmende Atemnot, } \\
\text { Atemstillstand }\end{array}$ & Asthmaanfall, Lungenödem, Aspiration \\
\hline Herz/Kreislauf & $\begin{array}{l}\text { akuter Brustschmerz, ausgeprägte oder } \\
\text { zunehmende Kreislaufinsuffizienz, } \\
\text { Kreislaufstillstand }\end{array}$ & $\begin{array}{l}\text { Herzinfarkt, Angina pectoris, Akutes } \\
\text { Koronarsyndrom (ACS), } \\
\text { Herzrhythmusstörungen, hypertone } \\
\text { Krise, Schock }\end{array}$ \\
\hline $\begin{array}{l}\text { Sonstige Schädigungen } \\
\text { mit Wirkung auf die } \\
\text { Vitalfunktionen }\end{array}$ & $\begin{array}{l}\text { schwere Verletzung, schwere Blutung, } \\
\text { starke akute Schmerzen, akute } \\
\text { Lähmungen }\end{array}$ & $\begin{array}{l}\text { Thorax-/Bauchtrauma, SHT, größere } \\
\text { Amputationen, Ösophagus- } \\
\text { varizenblutung, Verbrennungen, } \\
\text { Frakturen mit deutlicher Fehl- } \\
\text { stellung, Pfählungsverletzungen, } \\
\text { Vergiftungen, Schlaganfall }\end{array}$ \\
\hline Schmerz & $\begin{array}{l}\text { akute starke und/oder zunehmende } \\
\text { Schmerzen }\end{array}$ & Trauma, Herzinfarkt, Kolik \\
\hline
\end{tabular}


Tabelle 2: Indikationskatalog der BÄK für den Notarzteinsatz bezogen auf den Notfallhergang (BÄK 2013).

Schwerer Verkehrsunfall mit Hinweis auf Verletzte

Sonstiger Unfall mit Schwerverletzten

Unfall mit Kindern

Brände/Rauchgasentwicklung mit Hinweis auf Personenbeteiligung

Explosionsunfälle mit Hinweis auf Personenbeteiligung

Thermische oder chemische Unfälle mit Hinweis auf Personenbeteiligung

Strom- oder Blitzunfälle

Ertrinkungs- oder Tauchunfälle oder Eiseinbruch

Einklemmung oder Verschüttung

Drohender Suizid

Sturz aus Höhe $(\geq 3 \mathrm{~m})$

Schuss-/Stich-/Hiebverletzungen im Kopf-, Hals- oder Rumpfbereich

Geiselnahme, Amoklage oder sonstige Verbrechen mit unmittelbarer Gefahr für Menschenleben

Unmittelbar einsetzende oder stattgefundene Geburt

Vergiftungen mit vitaler Gefährdung

\subsection{Potenzielle Nachteile einer niederschwelligen Alarmierung für den Notarzt}

In der jüngeren Vergangenheit wurden jedoch auch erstmals potentielle Nachteile diskutiert, die sich aus der niederschwelligen Alarmierung arztbesetzter Rettungsmittel ergeben könnten (Sefrin et al. 2007). Auch wenn die negativen Auswirkungen einer liberalen Alarmierungsstrategie bislang nur punktuell untersucht wurden, sind die bisher geäußerten Argumente nachvollziehbar und schlüssig:

Die optimale Behandlung vital bedrohter Notfallpatienten setzt bei den Notärzten eine ausreichende Erfahrung in klinischer Diagnostik, medikamentöser Therapie und manuellen Fähigkeiten voraus. Der zunehmende Anteil von nicht Notfallpatienten im Rettungsdienst führt dazu, dass selbst regelmäßig tätige Notärzte immer häufiger nicht mehr über die notwendige Routine verfügen, um z. B. polytraumatisierte Patienten oder vital bedrohte Kinder adäquat zu versorgen, da sie viel seltener mit diesen Situationen konfrontiert werden. Eine Auswertung von 82.000 bodengebundenen Notarzteinsätzen (Gries et al. 2005) ergab beispielsweise, dass (bei angenommenen 16 Einsätzen pro Monat) lediglich alle 3,7 Monate eine nichtreanimationsbedingte Intubation vorgenommen wird und die Versorgung eines polytraumatisierten Patienten sogar nur alle 14,5 Monate erfolgt. 
Nicht zwingend notwendige Notarzteinsätze sind auch deshalb kritisch zu diskutieren, weil dadurch der Notarzt von anderen ärztlichen Aufgaben abgehalten wird. Ein Großteil der Notärztinnen und Notärzte sind während ihrer Arbeitszeit nicht ausschließlich für den Rettungsdienst abgeordnet, sondern leisten die Einsätze in der Regel aus dem regulären Klinikalltag heraus.

Nicht zuletzt muss die Tatsache erwähnt werden, dass bei Fahrten mit Sondersignalen ein fünffach erhöhtes Unfallrisiko (Pietschmann 2001) und ein vierfach erhöhtes Todesrisiko für die Insassen von Einsatzfahrzeugen besteht (Stolpe et al. 2002). Der unbegründete Einsatz eines Notarztes setzt die Besatzung und die Teilnehmer im Straßenverkehr einer nicht unerheblichen Gefahr aus, die auf ein Minimum reduziert werden sollte.

Um der Diskussion über Notwendigkeit und Möglichkeiten einer gezielteren Alarmierung von arztbesetzten Rettungsmitteln eine Grundlage zu geben, ist eine Analyse des IstZustandes erforderlich, wie hoch der Anteil an indizierten bzw. nicht indizierten Notarzteinsätzen tatsächlich ist.

\subsection{Bisherige Bewertung der Notarzt-Indikation: der NACA-Score}

Zur Beurteilung der Indikation von Notarzteinsätzen wird oft der National Advisory Comitee for Aeronautics Score (NACA-Score) bzw. seine Häufigkeitsverteilung herangezogen. Der NACA-Score umfasst ein einfaches Einteilungssystem zur Einordnung der Erkrankungsoder Verletzungsschwere von Patienten in 7 Kategorien, mit kurzen allgemein-klinischen Definitionen.

Tabelle 3: NACA-Score (nach Weiss et al. 2001).

\begin{tabular}{cl}
\hline NACA-Score & Schweregrad des Notfalles \\
\hline I & Geringfügige Störung \\
III & Ambulante Abklärung \\
IV & Stationäre Behandlung \\
V & Akute Lebensgefahr nicht auszuschließen \\
VI & Akute Lebensgefahr \\
VII & Reanimation \\
\hline
\end{tabular}

Der NACA-Score wird obligat bei jedem Notfallpatienten erhoben und in jedem notärztlichen Protokoll dokumentiert. Der Score ist unabhängig von Messwerten, lässt sich sowohl bei Erkrankungen als auch Verletzungen anwenden und ist damit für die präklinische 
Notfallmedizin, in der nur auf eingeschränkte diagnostische Möglichkeiten zurückgegriffen werden kann, besonders geeignet (Weiss et al. 2001).

Hierdurch können auch große Patientenkollektive einfach und gegebenenfalls sogar retrospektiv ausgewertet werden, ohne dass weitere Informationen über den Patienten erforderlich sind. Es existieren jedoch zunehmend Hinweise auf Limitationen insbesondere bei der Bewertung von Notarztindikationen (Lackner und Altemeyer 2005).

Trotz der Einfachheit des NACA-Scores ist seine Aussagekraft bei der Beurteilung präklinischer Notfallpatienten limitiert, da diese Klassifizierung im hohen Maße von der subjektiven Beurteilung der Notfallsituation durch den Notarzt abhängig ist. Dies ergab eine Untersuchung von Schlechtriemen et al, die den NACA-Score von über 100.000 Notfallpatienten retrospektiv anhand von objektivierbaren Parametern validierten. Die Autoren gelangen zu der Schlussfolgerung, dass der NACA-Score "zur retrograden Beurteilung der Einsatzindikation alleine nicht geeignet ist" (Schlechtriemen et al. 2005b). Der NACA-Score ermöglicht daher keine Beurteilung eines Einsatzes hinsichtlich seiner Notarztindikation.

\subsection{Schema zur Beurteilung der Indikation von Notarzteinsätzen}

Grundlage der vorliegenden Arbeit ist ein Klassifikationssystem, mit dem Notarzteinsätze in "indiziert" oder "nicht indiziert" eingeteilt werden können. Als Anforderung an das Klassifikationssystem wurde gestellt, dass die zugrundeliegenden Daten originär ärztliche Maßnahmen abbilden, einfach zu erheben und objektiv zu interpretieren sind. Als Kriterien wurde die Gabe von Medikamenten während des Einsatzes (zum Zeitpunkt der Datenerhebung eine originär ärztliche Maßnahme) und die ärztliche Begleitung des Patienten in eine Klinik aus medizinischen Gründen zugrunde gelegt.

Ein Notarzteinsatz wurde dann als indiziert bewertet, wenn vom Notarzt entweder ein Medikament verabreicht wurde, oder aber eine notärztliche Transportbegleitung aus medizinischen Gründen erfolgte (die per definitionem nur von einem Arzt geleistet werden kann). War beides nicht der Fall, wurde der Notarzteinsatz als nicht indiziert bewertet.

\subsection{Fragestellung}

Basierend auf den genannten Überlegungen leiten sich folgenden Fragestellungen ab:

1. Wie hoch ist der Prozentsatz an Notarzteinsätzen, die nach den genannten Kriterien als "indiziert" bzw. "nicht indiziert" eingestuft werden?

2. Sind die Anteile der als indiziert bewerteten Notarzteinsatze bei primärer Alarmierung und bei Nachalarmierung des Notarztes signifikant unterschiedlich?

3. Sind die Anteile der als indiziert bewerteten Notarzteinsatze bei RTH-Einsätzen und bei NEF-Einsätzen signifikant unterschiedlich? 
4. Sind die Anteile der als indiziert bewerteten Notarzteinsatze bei Einsätzen während geöffneter Hausarztpraxen und Einsätzen außerhalb von Praxisöffnungszeiten signifikant unterschiedlich?

5. Ist es von der Berufserfahrung des Notarztes abhängig, wie häufig Notarzt-Einsätze als indiziert beurteilt werden?

6. Sind die Anteile der als indiziert bewerteten Notarzteinsatze bei psychiatrischen Notfällen und nicht psychiatrischen Notfällen signifikant unterschiedlich?

7. Sind die Anteile der als indiziert bewerteten Notarzteinsatze bei traumatologischen Notfällen und bei nicht traumatologischen Notfällen signifikant unterschiedlich?

8. Sind die Anteile der als indiziert bewerteten Notarzteinsatze bei pädiatrischen Notfällen und bei nicht pädiatrischen Notfällen signifikant unterschiedlich?

9. Besteht eine Korrelation zwischen dem NACA-Score und dem Anteil der als indiziert eingestuften Einsätze?

10. Welche sind die häufigsten Alarmierungsmeldungen für einen Notarzteinsatz und wie ist das Verhältnis von indizierten zu nicht indizierten Einsätzen bei diesen Meldungen?

11. Welche Alarmierungsmeldungen sind mit dem niedrigsten Anteil indizierter Notarzteinsätze assoziiert?

12. Sind die Anteile der als indiziert bewerteten Notarzteinsatze bei Patienten $\leq 65$ Jahren und bei Patienten $>65$ Jahren signifikant unterschiedlich? 


\section{Material und Methoden}

\subsection{Ethikvotum}

Die Ethikkommission erteilte ein positives Votum zur Durchführung dieser Arbeit (Antragsnummer: 10/3/16).

\subsection{Studiendesign und Datenerfassung}

Die Untersuchung ist eine prospektive, nicht interventionelle Beobachtungsstudie auf Grundlage einer standardisierten Befragung der Notärzte, ergänzt mit den Daten aus elektronisch gespeicherten Notarztprotokollen (RED - Rettungsdienst Einsatz Dokumentationsbogen) für komplementäre Information.

Die Datenerhebung erfolgte im Zeitraum von 13.02.2017 bis 01.01.2018. Innerhalb dieses Intervalls wurden 35 Notärzte aus der Klinik für Anästhesiologie befragt, die auf den beiden in Göttingen stationierten Notarzteinsatzfahrzeugen und dem Rettungshubschrauber Christoph 44 tätig waren.

Alle Notärzte wurden im Untersuchungszeitraum gebeten, auf einem Fragebogen (siehe Anhang) kurze Angaben zu jedem Einsatz zu machen.

Abbestellung durch die Leitstelle, Fehleinsätze und Situationen, in denen kein Patient vorzufinden war (Einsatz als nicht auffindbare Person bezeichnet) wurden nicht in die Datenerfassung eingeschlossen.

Um den Dokumentationsaufwand für die Notärzte zu minimieren, wurden nur solche Informationen abgefragt, die nicht obligatorisch aus den elektronischen Einsatzprotokollen hervorgehen. In Tabelle 4 sind die Angaben zusammengefasst, die von den Notärzten zu jedem Einsatz gemacht wurden. 
Tabelle 4: Angabe des Notarztes zu den Einsätzen.

Datum des Einsatzes

Einsatznummer

Alarmierungsstichwort

Primäre Alarmierung oder Nachalarmierung

Patient befördert oder Behandlung vor Ort

Falls befördert, die Zielklinik des Transportes

Falls befördert, Transport vom Notarzt begleitet

Falls begleiteter Transport, Transport begleitet aus medizinischen Gründen

Anhand der Einsatznummer konnten die Informationen der Fragebögen mit denen des elektronischen Notarztprotokolls nach Vorgaben der DIVI (Deutsche Interdisziplinäre Vereinigung für Intensiv- und Notfallmedizin) (Röhrig und Walcher 2014) zusammengefügt werden. In Tabelle 5 sind diejenigen Angaben aufgeführt, die hierzu den elektronischen Einsatzprotokollen entnommen wurden.

Tabelle 5: Ergänzende Information zum Notarzteinsatz.

\section{Durch den Notarzt gestellte Verdachtsdiagnose}

Gabe von Medikamenten

Alter und Geschlecht des Patienten

Eingabe zum NACA-Score

Alle erhobenen Daten für diese Arbeit wurden in pseudonymisierter Form elektronisch archiviert und zur statistischen Auswertung im Tabellenkalkulationsprogramm Microsoft Excel 2019 (Microsoft Corporation, Redmond, USA) aufbereitet.

\subsection{Kategorieneinteilung der erfassten Daten}

Für die Beantwortung der formulierten Fragestellungen wurden die generierten Daten in Kategorien eingestuft. Tabelle 6 gibt eine Übersicht von den erfassten Kategorien. 
Tabelle 6: Kategorieneinteilung der erhobenen Daten.

Alarmierung: Primäre Alarmierung oder Nachalarmierung

Einsatzmittel: RTH oder NEF

Alarmierungszeitpunkt: Während oder außerhalb regulärer Praxisöffnungszeiten

Erfahrung des Notarztes: Erste Rotation im Notarztdienst oder bereits vorhandene

Erfahrung im Rettungsdienst

Psychiatrische oder nicht psychiatrische Notfälle

Traumatologische oder nicht traumatologische Notfälle

Pädiatrische oder nicht pädiatrische Notfälle

NACA-Score

Patientenalter $\leq 65$ Jahre oder $>65$ Jahre

Transportbegleitung: Erfolgt oder nicht erfolgt (wenn ja: Aus medizinischer Indikation?)

Eine Erläuterung der Kategorien der Tabelle 6 folgt in den nächsten Absätzen.

Die zeitgleiche Alarmierung von Notarzt und RTW alleine aufgrund der Notfallmeldung wird als "primäre Alarmierung" betrachtet. Wurde der Notarzt jedoch erst angefordert, nachdem eine Person mit medizinischen Kenntnissen mit der Erstversorgung begonnen hatte (z. B. Rettungsassistent, Hausarzt), wird dies als "Nachalarmierung" gewertet.

Alle Einsätze, bei denen der Notarzt mittels eines bodengebundenen Fahrzeuges zur Einsatzstelle gebracht wurde, werden als "NEF" gewertet. Alle anderen Fällen, d. h. Transport des Notarztes mit dem Rettungshubschrauber, werden als "RTH" kategorisiert.

Als gängige Praxisöffnungszeiten in Göttingen wurde der Zeitraum von 08:00 bis 18:00 Uhr an normalen Werktagen festgelegt. Einsätze während dieser Zeit werden als "während regulärer Praxisöffnungszeiten" kategorisiert, alle anderen Einsätze (inkl. am 24. und 31.12.) als "außerhalb regulärer Praxisöffnungszeiten".

Im Rettungsdienst der Stadt Göttingen werden Notärzte aus der Abteilung für Anästhesiologie der Universitätsmedizin Göttingen mit Facharztstandard bzw. ab dem vierten Weiterbildungsjahr eingesetzt. Als „Notärzte ohne Vorerfahrung“ wurden alle Notärzte kategorisiert, die ihre erste Rotation im Rettungsdienst absolvierten und über keine Vorerfahrung in der präklinischen Rettungsmedizin verfügen. Diese erste Rotation im Notarztdienst umfasst den Einsatz für vier Monate in Vollzeit auf dem NEF und für weitere sechs Monate auf dem RTH. Notärzte, die nach Abschluss dieser Rotation erneut im Rettungsdienst eingesetzt wurden, oder die über einen vergleichbaren Erfahrungshorizont 
aus Tätigkeiten an anderen Standorten verfügten, wurden der Kategorie „Notärzte mit Vorerfahrung" zugeordnet.

Als psychiatrische Notfälle wurden alle Einsätze klassifiziert, bei denen psychiatrische Symptome zur Entsendung des Notarztes geführt haben. Als traumatologische Notfälle wurden alle Einsätze klassifiziert, denen ein Trauma zugrunde lag. Als Kindernotfälle werden alle Einsätze klassifiziert, bei denen der Patient jünger als 16 Jahre war.

Die Einteilung "Patiententransport" vs. "kein Patiententransport" sowie (im Falle eines Transportes) die Einteilung in "mit Notarztbegleitung" vs. "ohne Notarztbegleitung" erfolgte anhand der Einsatzprotokolle. Für den Fall eines Transportes mit Notarztbegleitung erfolgte eine dritte Einteilung in "Transportbegleitung aus medizinischer Indikation" und

"Transportbegleitung ohne medizinische Indikation". Beispiele für eine Transportbegleitung ohne medizinische Indikation wären z. B. die Vervollständigung der Dokumentation auf der Fahrt oder die Rückkehr zur Notarztwache auf dem Gelände des Krankenhauses. Diese Klassifikation beruhte auf der individuellen Einschätzung des behandelnden Notarztes.

Der obligat bei jedem Einsatz erhobene NACA-Score wurde dem elektronischen Einsatzprotokoll entnommen.

Ein NACA-Score von VII wurde in unserer Arbeit zur Auswertung nicht betrachtet, da in diesem Falle die beiden Kriterien für die medizinische Indikation des Notarztes (Medikamentengabe und Transport des Patienten) keine Anwendung finden.

\subsection{Kriterien der medizinischen Indikation für den Notarzteinsatz}

Wie bereits beschrieben, wurde ein Einsatz als indiziert gewertet, wenn vom Notarzt entweder ein Medikament verabreicht wurde, oder der Notarzt den Patiententransport aus medizinischer Indikation begleitete.

Sauerstoff und kristalloide Infusionslösungen zählen zwar rechtlich betrachtet als Medikamente, sie werden jedoch regelmäßig von Rettungsassistenten und Notfallsanitätern eigenverantwortlich und ohne Nachforderung des Notarztes eingesetzt. Aus diesem Grunde wurde die Sauerstoffapplikation durch den Notarzt nicht als Medikamentengabe im Sinne dieser Studie gewertet, und die Gabe von kristalloiden Infusionslösungen nur dann, wenn sie nicht nur zum Offenhalten eines venösen Zuganges erfolgte, sondern explizit der Behandlung eines therapiebedürftigen Volumenmangels diente.

Neben der Gabe von Medikamenten kommen bei der präklinischen Versorgung regelmäßig viele andere Maßnahmen zur Anwendung, die ausschließlich ärztlichem Personal vorbehalten sind, wie z. B. Reanimation, Intubation, Kardioversion oder Defibrillation, Schrittmachertherapie oder nicht invasive Beatmung. Da diese ärztlichen Maßnahmen jedoch nahezu immer mit der Gabe von Medikamenten einhergehen, wurden sie nicht im 
Fragebogen erfasst, da die Entsendung des Notarztes in diesen Fällen nahezu immer über die Medikamentengabe als "indiziert" klassifiziert wurde.

In dem (unwahrscheinlichen) Fall, dass der Notarzt zwar eine lebensrettende ärztliche Maßnahme durchgeführt hat, aber kein Medikament verabreicht wurde und auch kein Transport erfolgte, wäre dieser Einsatz fälschlicherweise als "nicht indiziert" klassifiziert worden. Beispiele wären eine akute Dyspnoe durch die Verlegung einer Trachealkanüle, die vor Ort durch den Notarzt behoben werden kann, so dass der Patient zu Hause bleibt. Um bei Konstellationen dieser Art die Indikation des Notarzteinsatzes gegebenenfalls zu korrigieren, wurden die Notarztprotokolle von allen Einsätzen gründlich überprüft, bei denen die Entsendung des Notarztes nach den Kriterien dieser Untersuchung nicht indiziert war. Ergaben sich Hinweise darauf, dass andere, relevante ärztliche Maßnahmen zur Anwendung kamen, wurde der Notarzteinsatz als "indiziert" eingestuft.

Im Fall der Ausstellung einer Todesbescheinigung ohne weitere medizinische Maßnahmen (und ohne Transport) wurden die Einsätze als medizinisch indiziert gezählt.

\subsection{Datenschutz}

Die erhobenen Daten der Patienten (Alter, Geschlecht) sowie Informationen über den Einsatz wurden den Fragebögen und Dokumentationsprotokollen entnommen, die Datenerfassung und Auswertung erfolgte pseudonymisiert mit Hilfe der universellen Einsatznummer. Es wurden im Rahmen dieser Studie keine personenbezogenen Daten erfragt oder verarbeitet.

\subsection{Statistische Verfahren}

Die deskriptive und statistische Auswertung der Daten erfolgte mit Hilfe von SPSS Statistics (SPSS Inc., Chicago, IL, USA) und Excel 2019 (Microsoft Corporation, Redmond, USA). Die Häufigkeiten bestimmter Ergebnisse wurde in absoluten Zahlen und als prozentualer Anteil beschrieben. Zur Untersuchung von Unterschieden zwischen einzelnen kategorialen Variablen wurde der T- Test nach Student angewendet. Zur Signifikanztestung der verschiedenen Gruppen der kategorialen Variablen wurde den Pearsons-Chi-Quadrat-Test eingesetzt. Der Spearmans Korrelationskoeffizient wurde bei bivariater Analyse angewendet. Eine Fehlerwahrscheinlichkeit von $\mathrm{p}<0,05$ wurde als statistisch signifikant betrachtet.

\subsection{Literaturrecherche und Verwaltung der bibliographischen Quellen}

Es erfolgte eine selektive Literaturrecherche in den medizinischen Datenbanken PubMed (U.S. National Library of Medicine), Science Direct (Elsevier B.V.) sowie Google Scholar 
(Google LLC) und Springer Link (Spinger Nature Switzerland AG), mit Hilfe von Referenzen aus relevanten Publikationen sowie unter Einbeziehung aktueller themenrelevanter Richtlinien, Leitlinien und Empfehlungen.

Die Verwaltung der wissenschaftlichen bibliographischen Referenzen sowie die Erstellung des Literaturverzeichnisses fand mit Anwendung der Software Zotero (George Mason University, Virginia, USA) statt. 


\section{Ergebnisse}

Insgesamt wurden im Untersuchungszeitraum Daten von 3067 Notarzteinsätzen erfasst. 64 Einsätze mussten wegen fehlender Informationen ausgeschlossen werden, so dass 3003 vollständige Datensätze generiert und in dieser Arbeit untersucht werden konnten.

Von 3003 Patienten im gesamten Beobachtungszeitraum wurden 2494 (83,05\%) in ein Krankenhaus befördert, 385 (12,82\%) wurden vor Ort ambulant behandelt, und bei 124 $(4,12 \%)$ Einsätzen starben Patienten an der Einsatzstelle.

\subsection{Verhältnis indiziert und nicht indizierter Notarzteinsätze}

Von den insgesamt 3003 ausgewerteten Notarzteinsätzen war bei 2131 Einsätzen (71\%) die Indikation zur Alarmierung des Notarztes nach den Kriterien dieser Untersuchung gegeben, während bei 872 Fällen (29\%) keine Indikation nach den genannten Kriterien gegeben war. Dieser Unterschied war statistisch signifikant $(\mathrm{p}<0,0001)$.

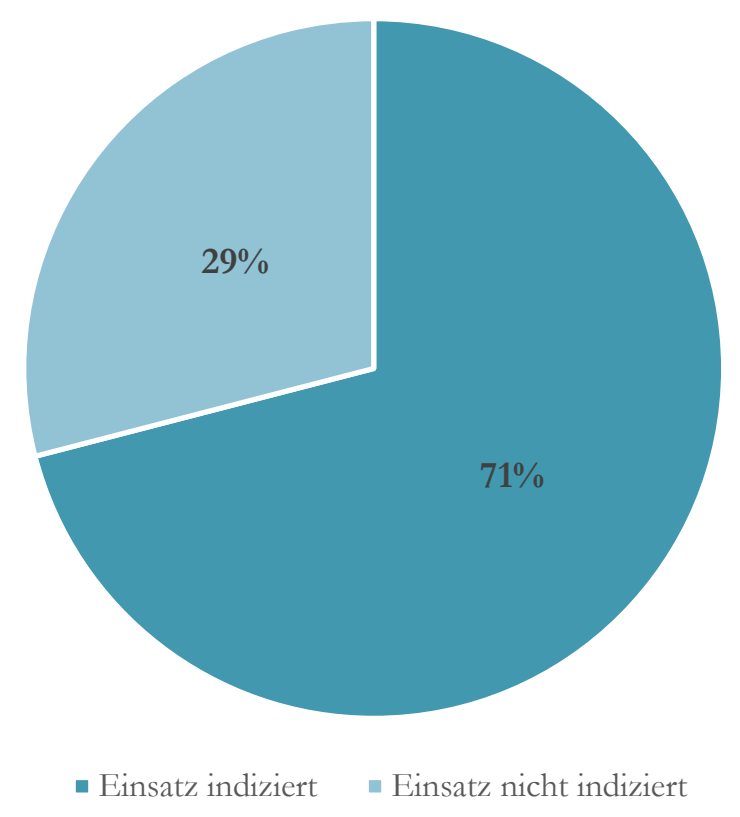

Abbildung 4: Verteilung der gesamten Notarzteinsätze hinsichtlich des Bestehens einer medizinischen Indikation (in Prozent).

\subsection{Demographische Verteilung}

Der Anteil der männlichen Patienten war mit 53,94\% $(\mathrm{n}=1620)$ minimal größer als der Anteil weiblicher Patienten, dieser betrug 46,06\% ( $\mathrm{n}=1383$ ).

Das Alter der behandelten Patienten reichte von 0 bis 106 Jahren. Bei der Altersverteilung war eine starke Zunahme der relativen Häufigkeit ab einem Alter von 50 Jahre zu 
verzeichnen, $71,32 \%$ der Patienten waren über 50 Jahre alt. Der Gipfel der Häufigkeitsverteilung liegt bei einem Patientenalter zwischen 70 bis 80 Jahren.

Die Gruppe der Patienten über 65-jährigen umfasste 1570 Patienten, innerhalb dieser Gruppe wurden 74,65\% der Einsätze als indiziert bewertet $(\mathrm{n}=1172)$. Die Gruppe der Patienten bis 65 Jahre umfasste 1433 Patienten, bei denen die Indikation für den Notarzteinsatz in lediglich 66,92\% der Fälle gegeben war $(\mathrm{n}=959)$. Dieser Unterschied in der Häufigkeit indizierter Notarzteinsätze war statistisch signifikant $(p=0,0001)$.

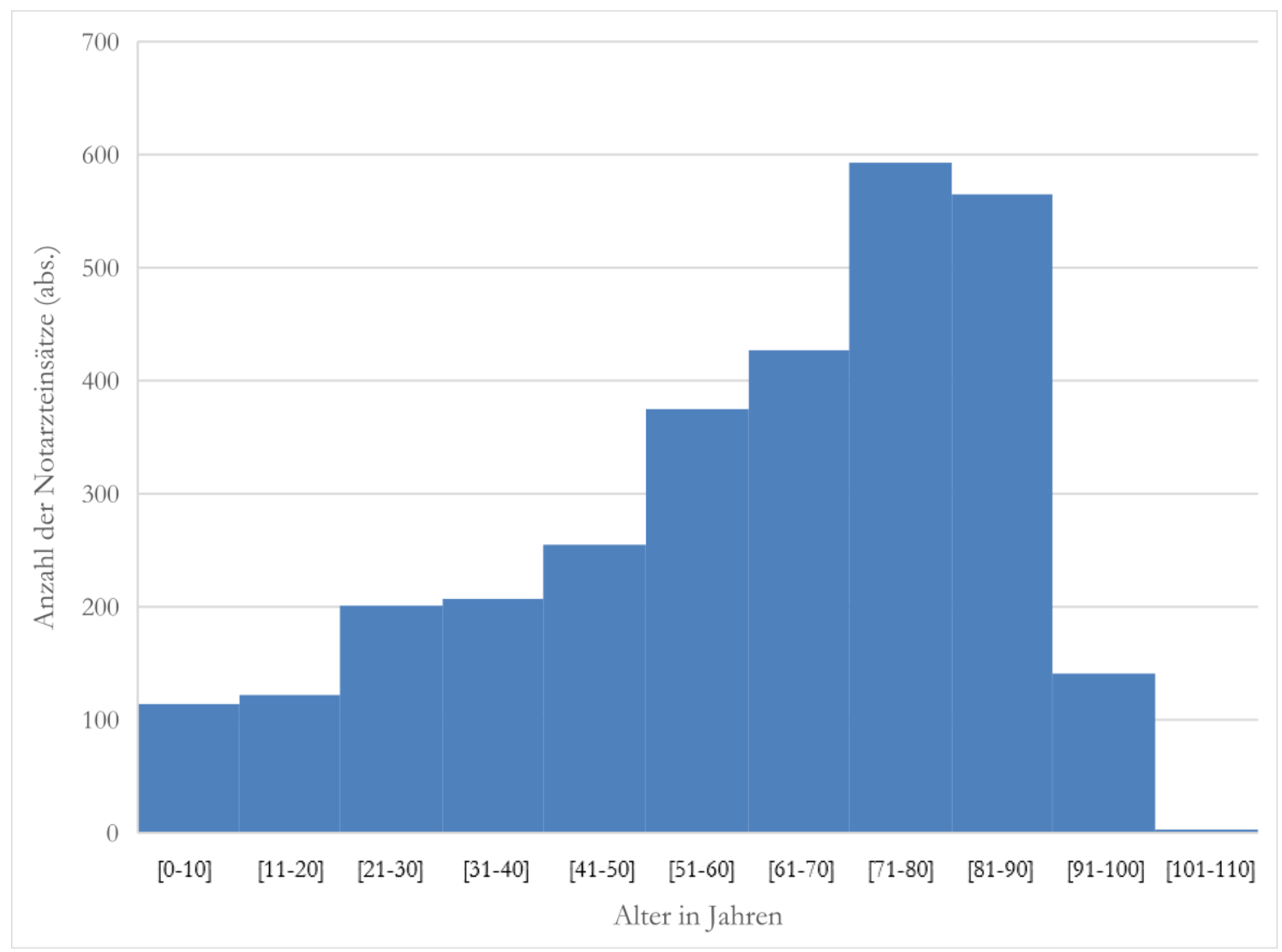

Abbildung 5: Altersverteilung der behandelten Patienten.

\subsection{Die zwanzig häufigsten Alarmierungsmeldungen}

In Tabelle 7 und Abbildung 6 sind die 20 häufigsten Alarmierungsmeldungen und ihre Häufigkeiten zusammengefasst. Diese 20 häufigsten Meldungen kamen in 2550 der insgesamt 3003 Notarzteinsätze vor und deckten damit 84,9\% der ausgewerteten Einsätze ab. Die mit Abstand am häufigsten vorkommende Meldung ist das ,akute Koronarsyndrom“, gefolgt von der „nicht ansprechbaren Person“ und ,akuter Atemnot“. 
Tabelle 7: Die zwanzig häufigsten Einsatzmeldungen und Angabe der jeweiligen med. Indikation.

\begin{tabular}{|c|c|c|c|}
\hline & Alarmierungsmeldung & $\begin{array}{l}\text { Häufigkeit der Einsätze } \\
\text { abs. (in \%) }\end{array}$ & $\begin{array}{l}\text { Anteil indizierte Einsätze } \\
\text { abs. (in \%) }\end{array}$ \\
\hline 1. & ACS & $415(13,8 \%)$ & $342(82 \%)$ \\
\hline 2. & NAP & $332(11,1 \%)$ & $182(55 \%)$ \\
\hline 3. & Luft-/Atemnot/Dyspnoe & $245(8,2 \%)$ & $181(74 \%)$ \\
\hline 4. & Krampfanfall & $229(7,6 \%)$ & $116(51 \%)$ \\
\hline 5. & Treppe-/Fahrradsturz & $154(5,1 \%)$ & $126(82 \%)$ \\
\hline 6. & COPD & $140(4,7 \%)$ & $120(56 \%)$ \\
\hline 7. & $\mathbf{V U}+\mathbf{M U}$ & $119(4,0 \%)$ & $98(82 \%)$ \\
\hline 8. & Apoplex & $103(3,4 \%)$ & $73(71 \%)$ \\
\hline 9. & BAP & $82(2,7 \%)$ & $44(54 \%)$ \\
\hline 10. & Kindernotfall & $76(2,5 \%)$ & $49(64 \%)$ \\
\hline 11. & Thorax-/Burstschmerzen & $74(2,5 \%)$ & $53(71 \%)$ \\
\hline 12. & Hypertonus/Hypertensive Entgleisung & $71(2,4 \%)$ & $60(85 \%)$ \\
\hline 13. & Analgesie & $61(2,0 \%)$ & $56(92 \%)$ \\
\hline 14. & Sekundärverlegung & $60(2,0 \%)$ & $55(92 \%)$ \\
\hline 15. & Reanimation & $57(1,9 \%)$ & $41(72 \%)$ \\
\hline 16. & HRST/Tachykardie/Bradykardie & $47(1,6 \%)$ & $35(74 \%)$ \\
\hline 17. & Tabletten-/ Medikamentenintoxikation & $46(1,5 \%)$ & $34(74 \%)$ \\
\hline 18. & Allergische/Anaphylaktische Reaktion & $44(1,5 \%)$ & $37(84 \%)$ \\
\hline 19. & Abdominelle Schmerzen/Akutes Abdomen & $32(1,1 \%)$ & $22(69 \%)$ \\
\hline 20. & Suizid-ankündigung/Androhung/Versuch & $29(1,0 \%)$ & $14(48 \%)$ \\
\hline
\end{tabular}




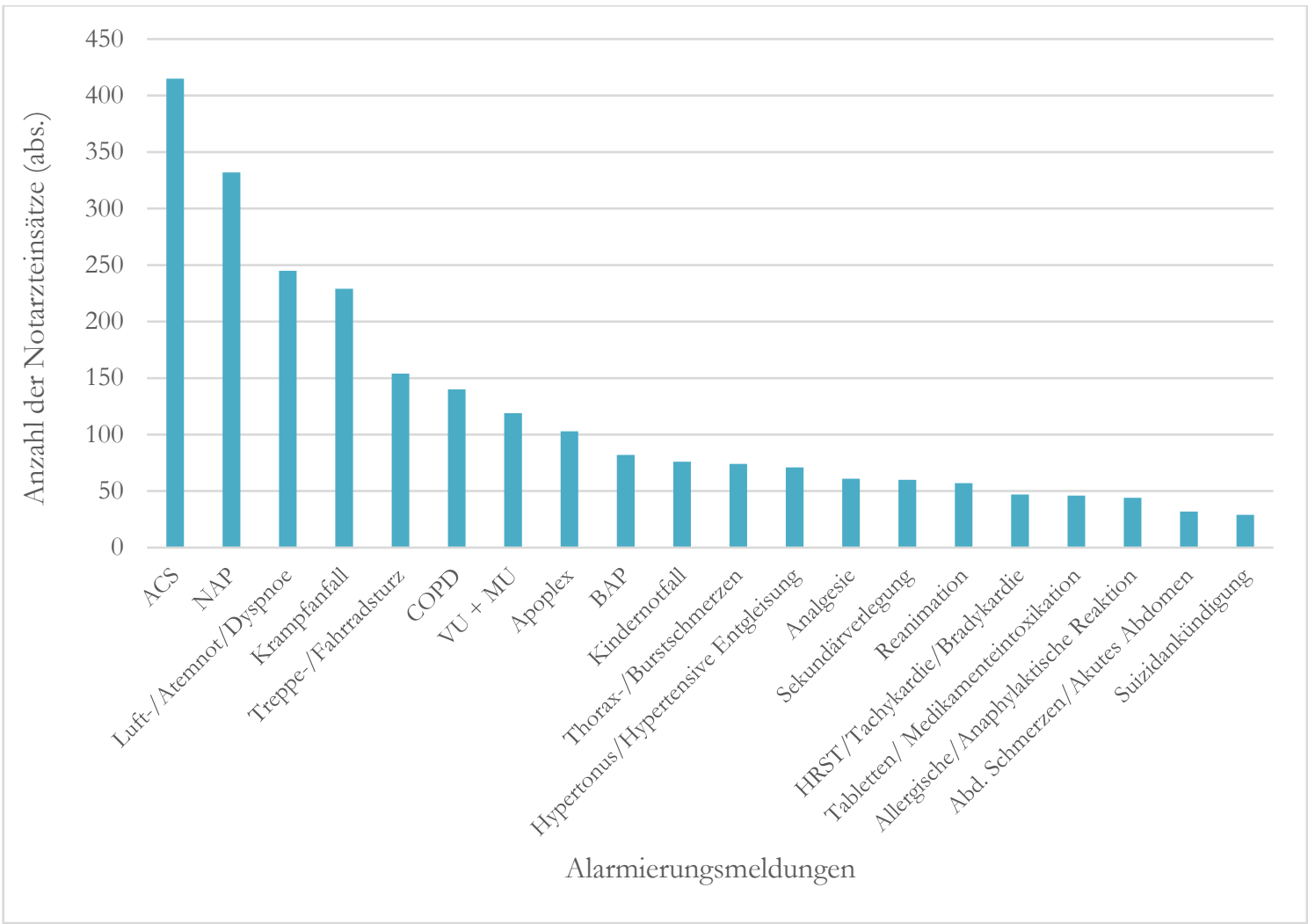

Abbildung 6: Häufigkeitsverteilung der 20 häufigsten Alarmierungsmeldungen.

Die zehn häufigsten Einsatzmeldungen deckten 1895 (63\%) aller Einsätze ab.

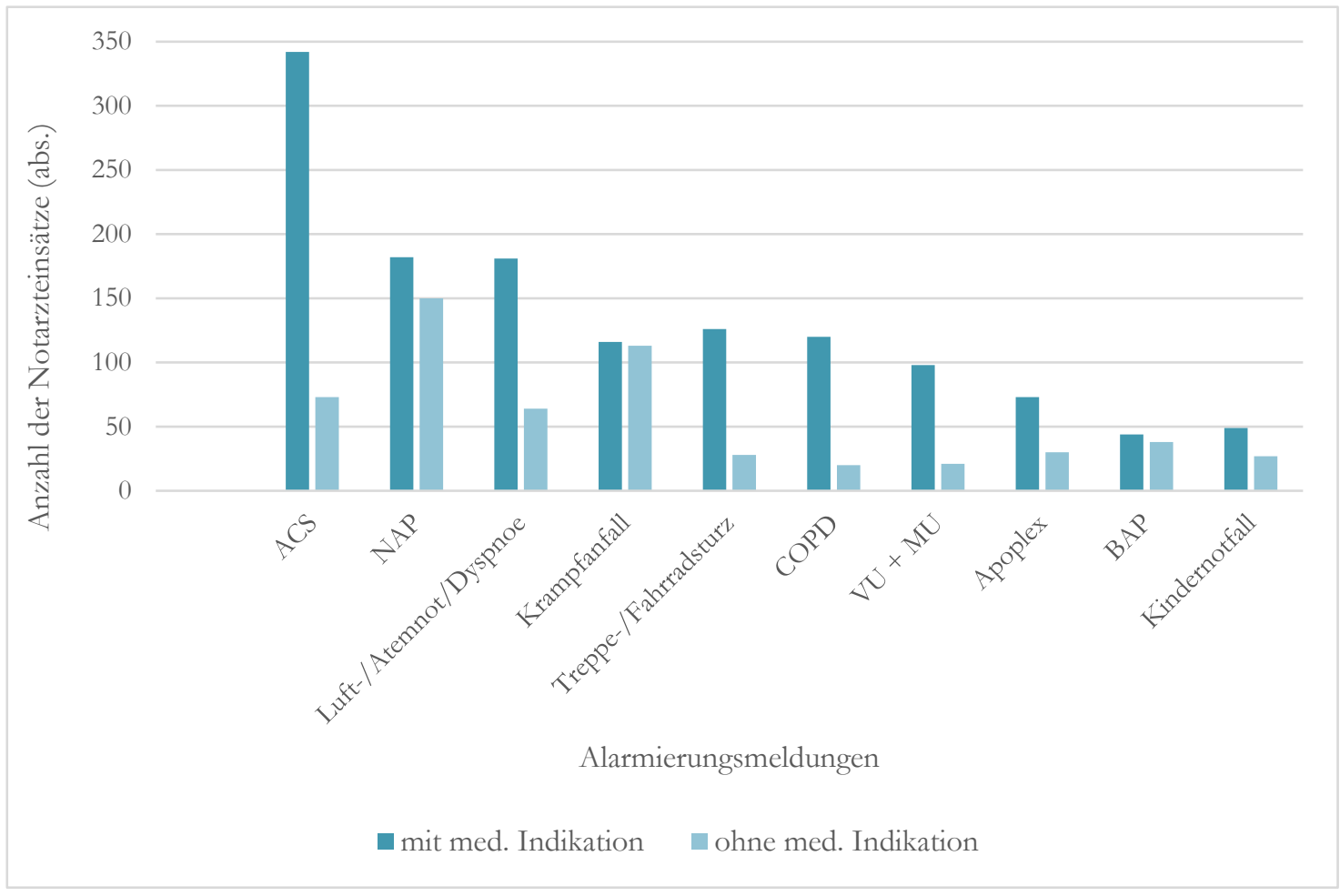

Abbildung 7: Häufigkeitsverteilung der zehn häufigsten Einsatzmeldungen. 


\subsection{Die zehn Alarmierungsmeldungen mit dem höchsten Anteil einer gegebenen Notarztindikation}

Die Alarmierungsmeldungen mit den zehn höchsten Anteilen indizierter Notarzteinsätze entfielen auf insgesamt 1335 Einsätze (44\%). Die höchste Rate indizierter Notarzteinsätze trat bei den Einsatzstichworten "Analgesie" (92\%) und "Sekundärverlegung" (92\%) auf, gefolgt von "COPD" (86\%), "hypertensive Entgleisung" (85\%) und "allergische/anaphylaktische Reaktion" mit 84\% indizierter Notarztalarmierungen. Die Alarmierungsmeldungen "ACS", "Verkehrs- oder Motorradunfall" sowie "Sturz" waren in $82 \%$ der Fälle mit einer gegebenen Notarztindikation assoziiert, "Hypoglykämie" in 81\% und "Atemnot/Dyspnoe" in 76\% der Fälle.

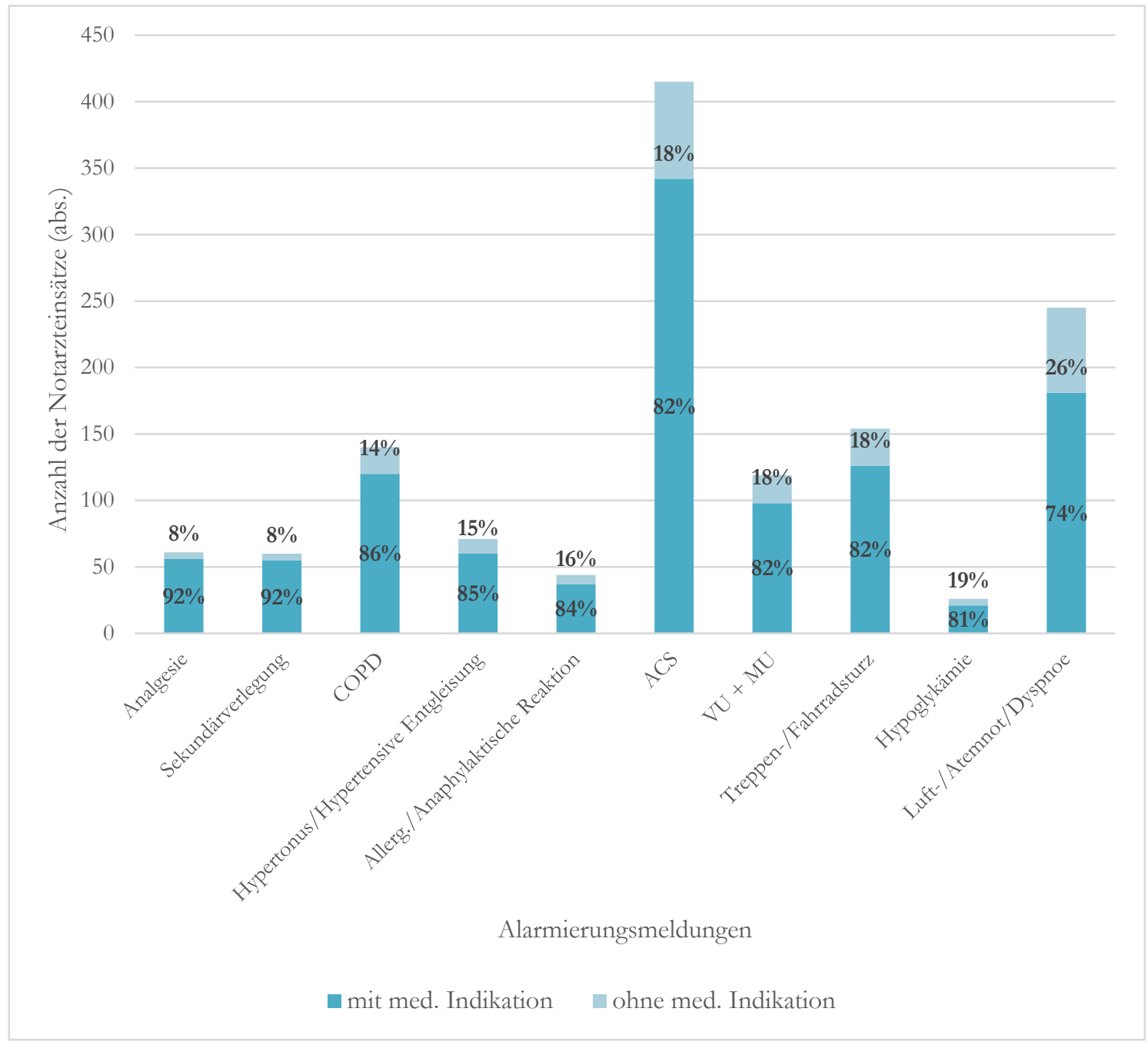

Abbildung 8: Zehn Alarmierungsmeldungen mit dem höchsten Anteil indizierter Notarztalarmierungen. Für jede Alarmierungsmeldung ist der prozentuale Anteil der Einsätze mit und ohne medizinische Indikation aufgeführt. 


\subsection{Die zehn Alarmierungsmeldungen mit dem niedrigsten Anteil einer gegebenen Notarztindikation}

Die Alarmierungsmeldung mit dem niedrigsten Anteil einer gegebenen Notarztindikation war „psychischer Ausnahmezustand“; hier war der Einsatz des Notarztes in nur 27\% der Fälle gerechtfertigt. Weitere Meldungen mit niedrigem Anteil indizierter Notarztbeteiligung (Prozentwerte in Klammern) waren Suizidankündigung (48\%), Krampfanfall (51\%), bedingt ansprechbare Person und nicht ansprechbare Person (54\% und $55 \%$ ). Beim Einsatzstichwort "Kindernotfall" waren 64\% der Notarzteinsätze indiziert, bei der Alarmierung "kollabierte Person" 66\%, bei der Meldung "akutes Abdomen" 69\% und bei "Apoplex" und "thorakaler Schmerz" jeweils 71\%.

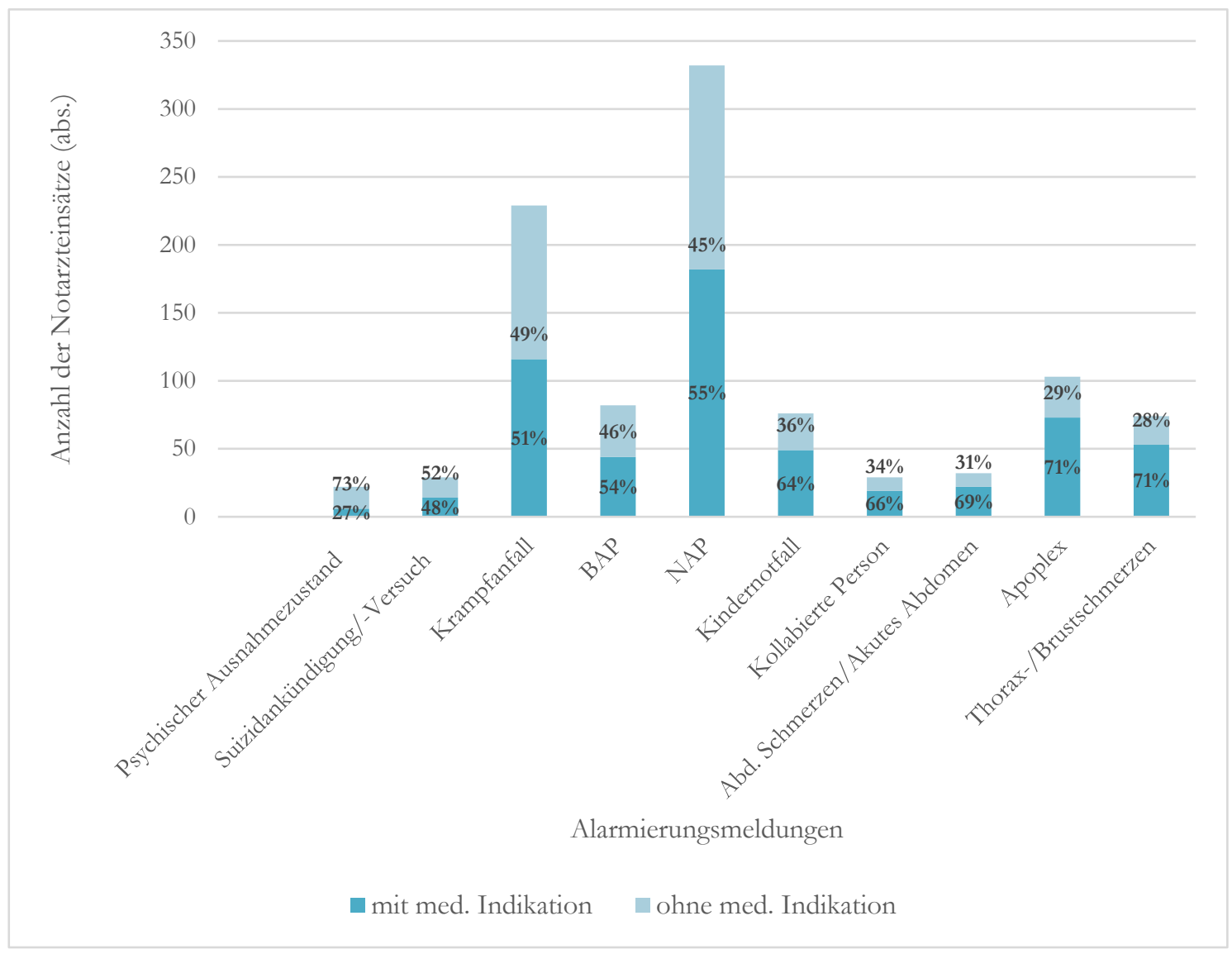

Abbildung 9: Zehn Einsatzmeldungen mit dem niedrigsten Anteil einer medizinischen Indikation. Für jede Alarmierungsmeldung ist der prozentuale Anteil der Einsätze mit und ohne medizinische Indikation aufgeführt. 


\subsection{Anteil medizinisch indizierter Notarzteinsätze bei primärer Alarmierung vs. Nachalarmierung}

Bei 2383 Einsätzen (79\%) wurde der Notarzt bereits aufgrund der Notfallmeldung zeitgleich mit dem RTW alarmiert ( = primäre Alarmierung), bei den restlichen 620 Einsätzen (21\%) wurde der Notarzt von den erstversorgenden Kräften nachalarmiert.

Im Falle der Erstalarmierung betrug der Anteil von medizinisch indizierten Notarzteinsätzen $68 \%(\mathrm{n}=1616)$, bei 32\% $(\mathrm{n}=767)$ lag keine Indikation für den Notarzteinsatz vor.

Im Falle einer Nachalarmierung war eine medizinische Indikation nach den Kriterien dieser Untersuchung dagegen in 83\% $(n=515)$ der Fälle gegeben und in lediglich 17\% $(n=105)$ nicht.

Dieser Unterschied in der Häufigkeit indizierter Notarzteinsätze bei primärer Alarmierung und Nachalarmierung war statistisch signifikant $(p<0,001)$.

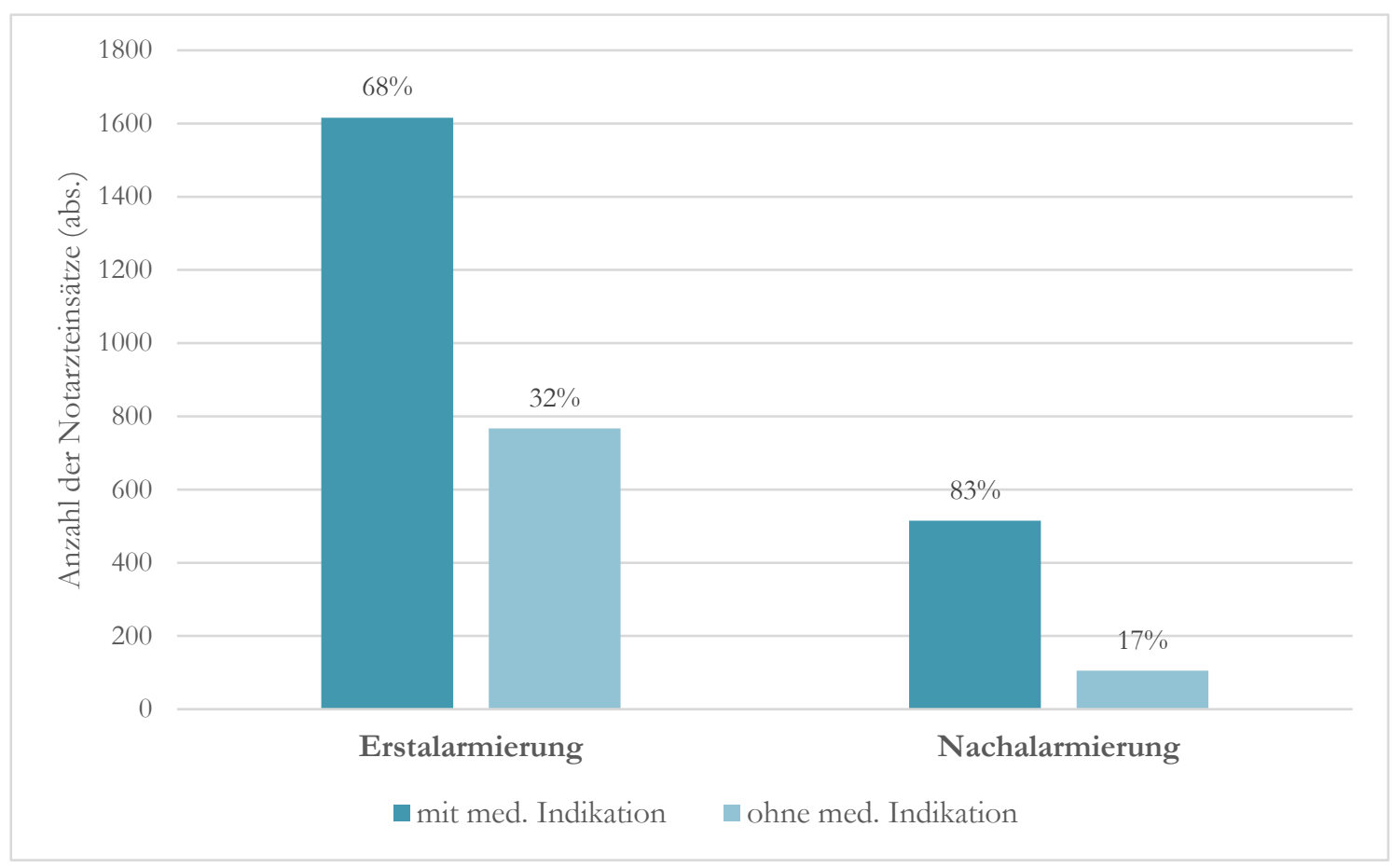

Abbildung 10: Erstalarmierung vs. Nachalarmierung in Abhängigkeit von der medizinischen Indikation.

\subsection{Anteil medizinisch indizierter Notarzteinsätze bei NEF- Einsätzen und RTH-Einsätzen}

Von allen 3003 ausgewerteten Einsätzen wurden 69\% ( $\mathrm{n}=2084)$ bodengebunden mittels eines Notarzteinsatzfahrzeuges (NEF 1-82-1 und NEF 2-82-1) und 31\% ( $\mathrm{n}=919$ ) luftgebunden mit dem Rettungshubschrauber Christoph 44 durchgeführt. 
Der Anteil medizinisch indizierter Notarztalarmierungen war in den beiden Gruppen unterschiedlich: In 69\% der NEF-Einsätze war die Indikation für den Notarzt nach den Kriterien dieser Studie gegeben, bei RTH-Einsätzen lag dieser Anteil bei 75\%. höher. Dieser Unterschied war statistisch signifikant $(p=0,004)$.

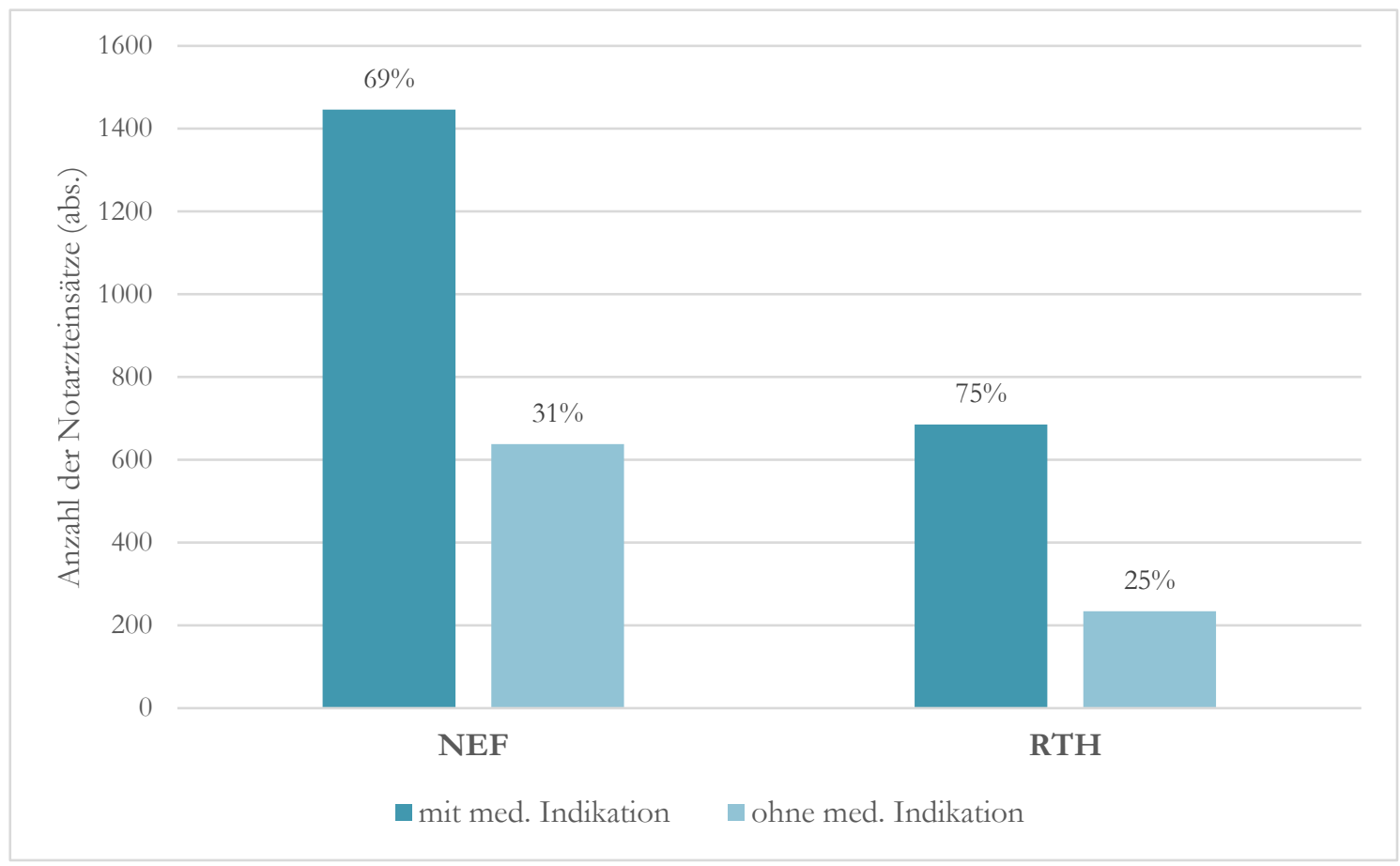

Abbildung 11: Graphik der Verteilung der medizinischen Indikation beim Einsatz des NEF vs. RTH.

\subsection{Anteil medizinisch indizierter Notarzteinsätze während und außerhalb regulärer Praxisöffnungszeiten}

Die Anzahl der Notarzteinsätze innerhalb der Öffnungszeiten von Hausarztpraxen betrug 1295 (43\%), außerhalb der Öffnungszeiten fanden 1708 Einsätze statt (57\%).

Der Anteil medizinisch indizierter Notarzteinsätze war während und außerhalb der Öffnungszeiten niedergelassener Ärzte nahezu gleich (70\% vs. 72\%), dieser Unterschied war statistisch nicht signifikant $(p=0,3)$. 


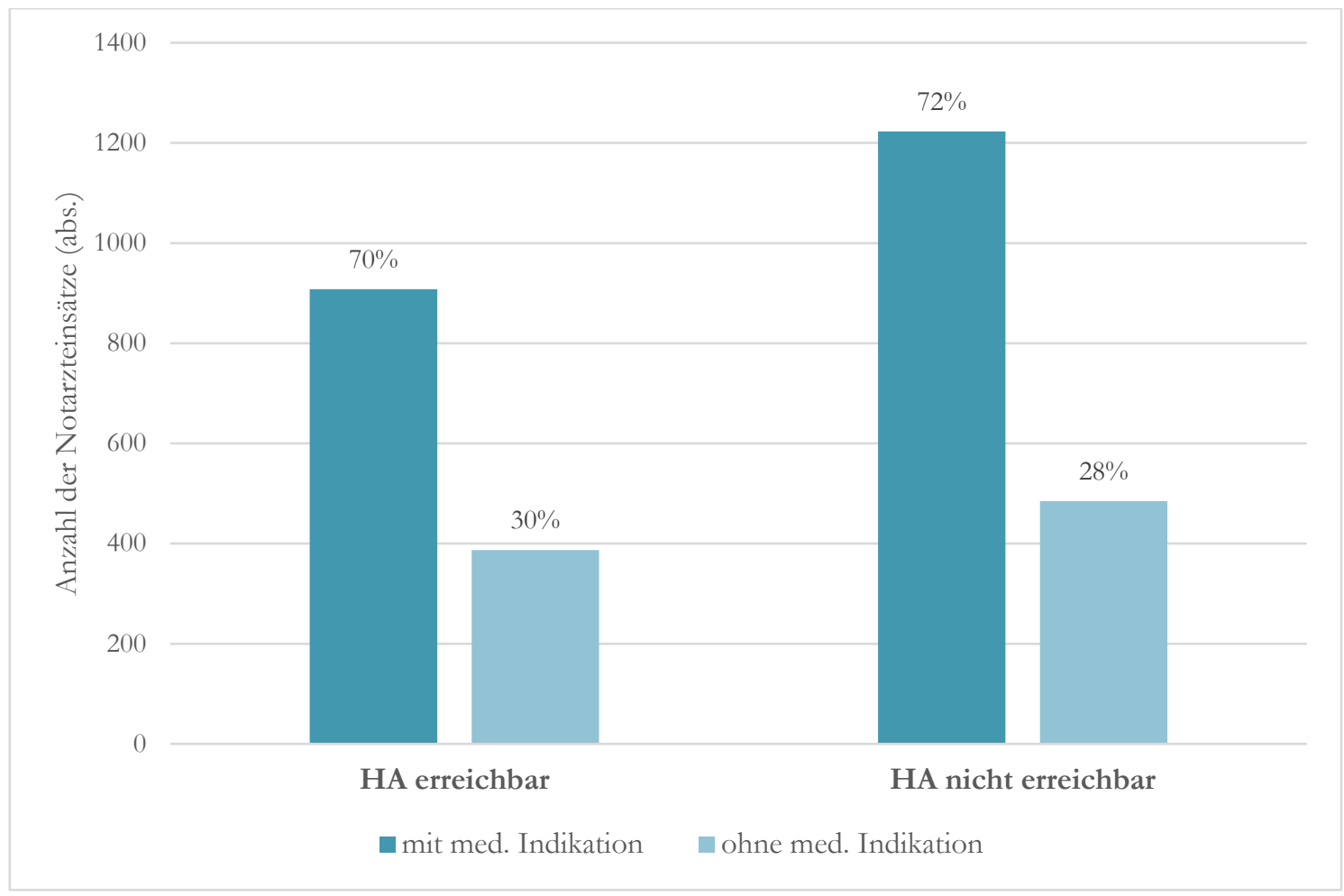

Abbildung 12: Darstellung des Anteils der indizierten Einsätze vs. ohne medizinische Indikation nach Hausarzterreichbarkeit.

\subsection{Anteil medizinisch indizierter Notarzteinsätze in den Gruppen von Notärzten mit und ohne Vorerfahrung im Rettungsdienst}

Im Untersuchungszeitraum der vorliegenden Studie nahmen insgesamt 34 Notärzte am Rettungsdienst der Stadt Göttingen teil. Sieben Ärzte (21\%) absolvierten ihre erste Rotation im Rettungsdienst und wurden daher der Gruppe "Keine Vorerfahrung im Rettungsdienst" zugeordnet. 27 Ärzte (79\%) verfügten bereits über Vorerfahrung im Rettungsdienst, die sie entweder an der Klinik für Anästhesiologie oder anderen Institutionen erworben hatten.

Die Gruppe der Ärzte ohne Vorerfahrung im Rettungsdienst absolvierte 1230 Einsätze (41\%), während bei 1773 Notfalleinsätzen (59\%) Kollegen mit Vorerfahrung zum Einsatz kamen. 


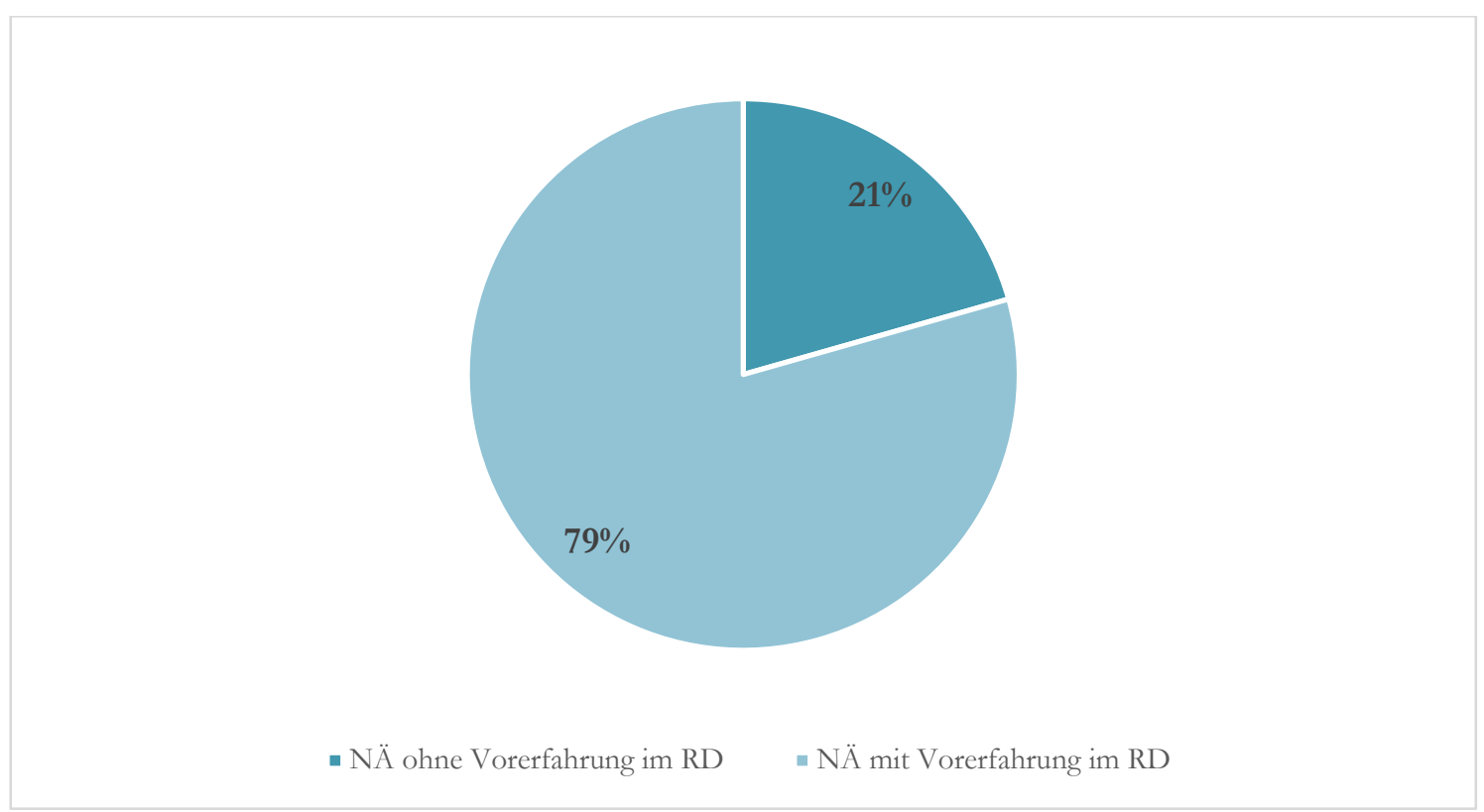

Abbildung 13: Anteil Notärzte ohne Vorerfahrung und mit Vorerfahrung im Ärzteteam in Göttingen (in Prozent).

Der Anteil an Einsätzen, die nach den genannten Kriterien als medizinisch indiziert eingestuft wurde, wies in den Gruppen der Notärzte mit und ohne Vorerfahrung im Rettungsdienst keinen signifikanten Unterschied auf: In der Gruppe von Ärzten mit Vorerfahrung wurden 68,12\% als indiziert klassifiziert, in der Gruppe ohne Vorerfahrung im Rettungsdienst 70,70\% der Einsätze $(p=0,45)$.

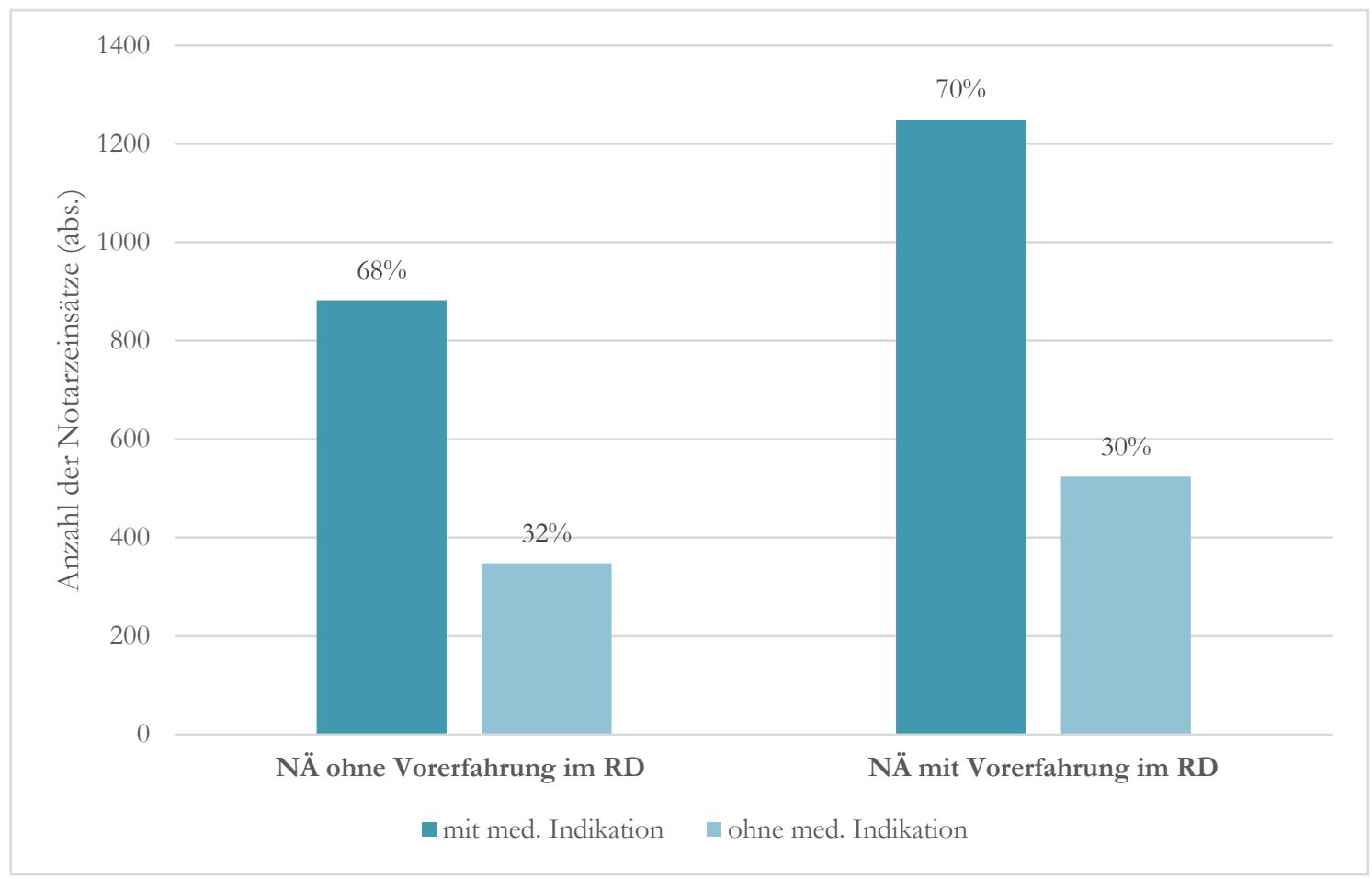

Abbildung 14: Graphische Verteilung Einsätze mit und ohne medizinische Indikation nach Erfahrung der NÄ. 
In 2494 der insgesamt 3003 Einsätze erfolgte ein Transport des Patienten in ein Krankenhaus. In der Gruppe der Notärzte ohne Vorerfahrung im Rettungsdienst erfolgte eine Transportbegleitung durch den Notarzt aus medizinischen Gründen in 79\% der Fälle, in der Gruppe der Notärzte mit Vorerfahrung im Rettungsdienst lag dieser Anteil mit 71\% niedriger. Dieser Unterschied war statistisch signifikant $(\mathrm{p}=0,0002)$.

Keinen signifikanten Unterschied in den beiden Gruppen gab es dagegen bei der Häufigkeit einer Medikamentengabe: Notärzte mit Vorerfahrung im Rettungsdienst verabreichten in $59 \%$ der Fälle ein oder mehrere Medikamente, in der Gruppe ohne Vorerfahrungen waren es $57 \%(p=0,59)$.

Ebenfalls kein signifikanter Unterschied bestand bei der Häufigkeit einer rein ambulanten Behandlung: Notärzte mit Vorerfahrung im Rettungsdienst beließen in 14\% der Fälle die Patienten nach der Behandlung vor Ort, in der Gruppe der Notärzte ohne Vorerfahrung im Rettungsdienst war dies in 12\% der Einsätze der Fall $(p=0,235)$.

\subsection{Anteil medizinisch indizierter Notarzteinsätze bei traumatologischen und nicht traumatologischen Einsätzen}

Von den gesamten Einsätzen entfielen 370 (12\%) auf traumatologische und 2633 (88\%) auf nicht traumatologische Notfälle.

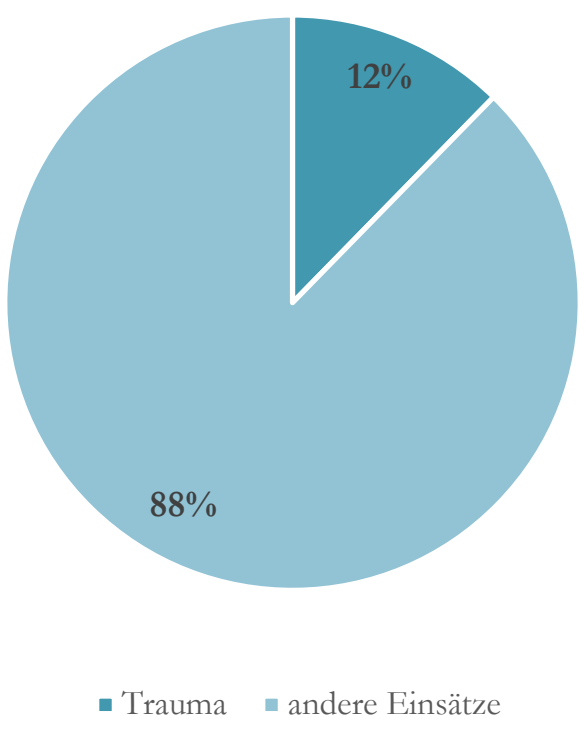

Abbildung 15: Anteil der traumatologischen Notfälle an den Gesamteinsatzmeldungen (in Prozent).

In der Gruppe der traumatologischen Notfälle war eine Indikation für die Entsendung des Notarztes in $82 \%(\mathrm{n}=305)$ der Fälle gegeben, bei den nicht traumatologischen Notfällen lag dieser Anteil lediglich bei 69\% $(\mathrm{n}=1826)$. Dieser Unterschied war statistisch signifikant $(\mathrm{p}$ $=0,0001)$. 


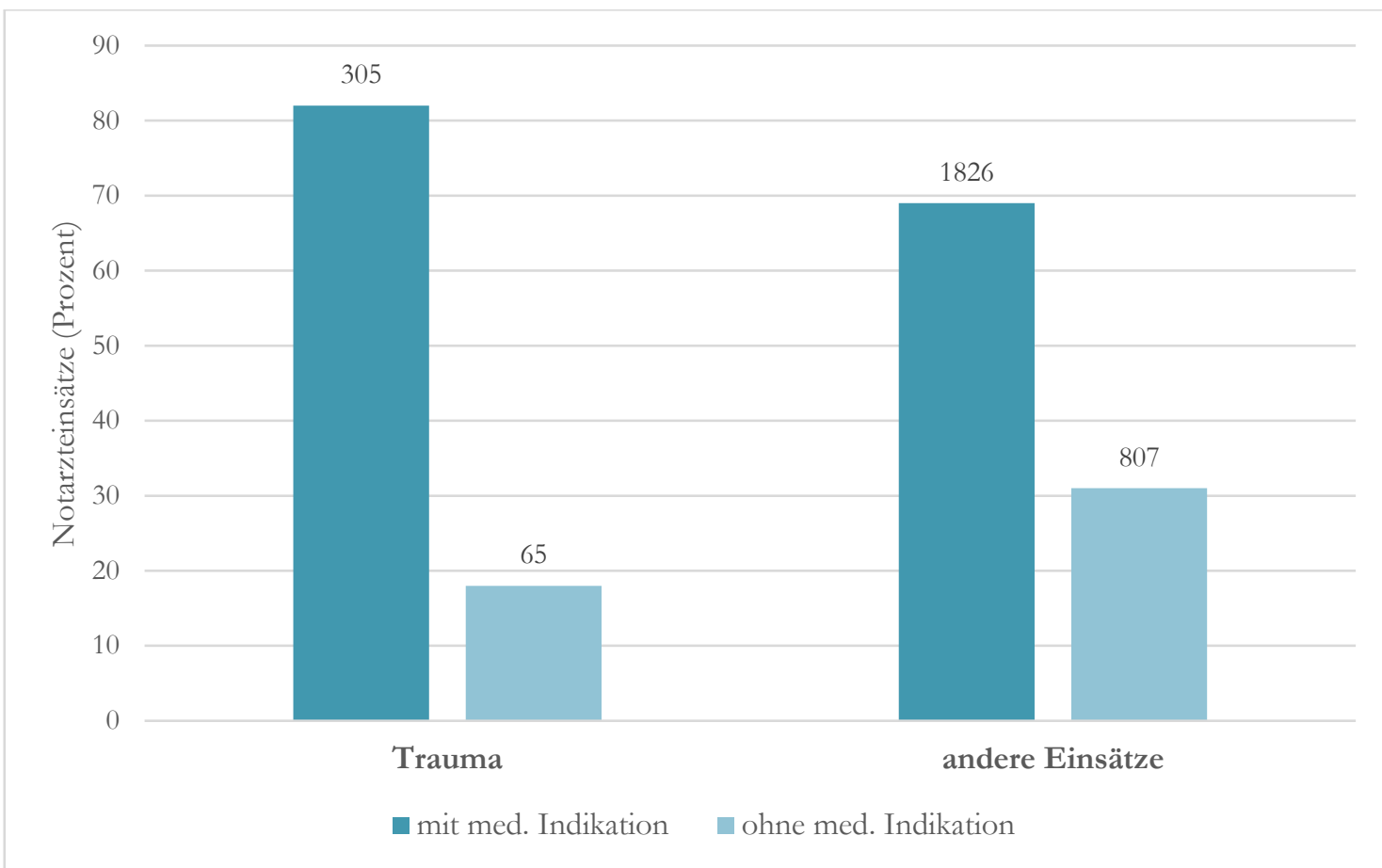

Abbildung 16: Prozentuale Verteilung des Vorliegens einer medizinischen Indikation bei traumatologischen vs. nicht traumatologischen Notfällen. Oberhalb der Balken befinden sich die entsprechenden absoluten Fallzahlen.

\subsection{Anteil medizinisch indizierter Notarzteinsätze in den Gruppen psychiatrische Notfälle und nicht psychiatrische Notfälle}

Im Untersuchungszeitraum fielen 58 Einsätze in die Kategorie psychiatrische Notfälle, dies entspricht $2 \%$ der gesamten Einsätze.

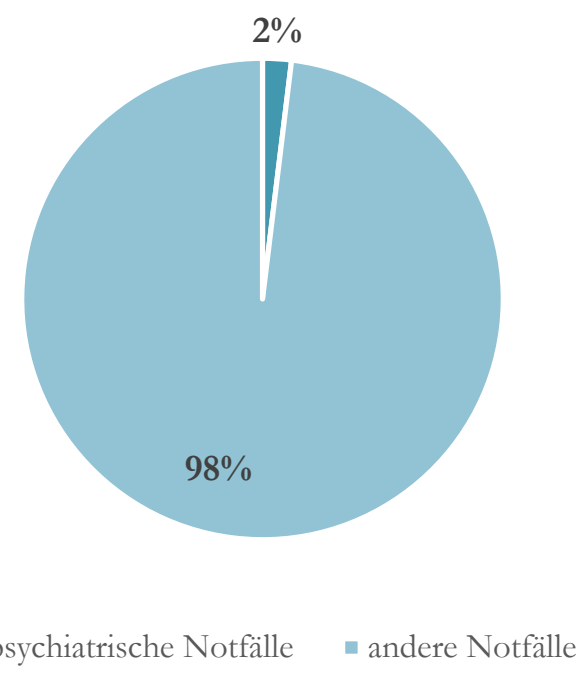

Abbildung 17: Anteil der psychiatrischen Notfälle an den Gesamteinsatzmeldungen (in Prozent). 
Bei psychiatrischen Notfällen war die Indikation für den Notarzt nur in 34\% der Fälle gegeben, bei den nicht psychiatrischen Notfällen dagegen in $72 \%$. Dieser Unterscheid erwies sich als statistisch signifikant $(\mathrm{p}=0,001)$.

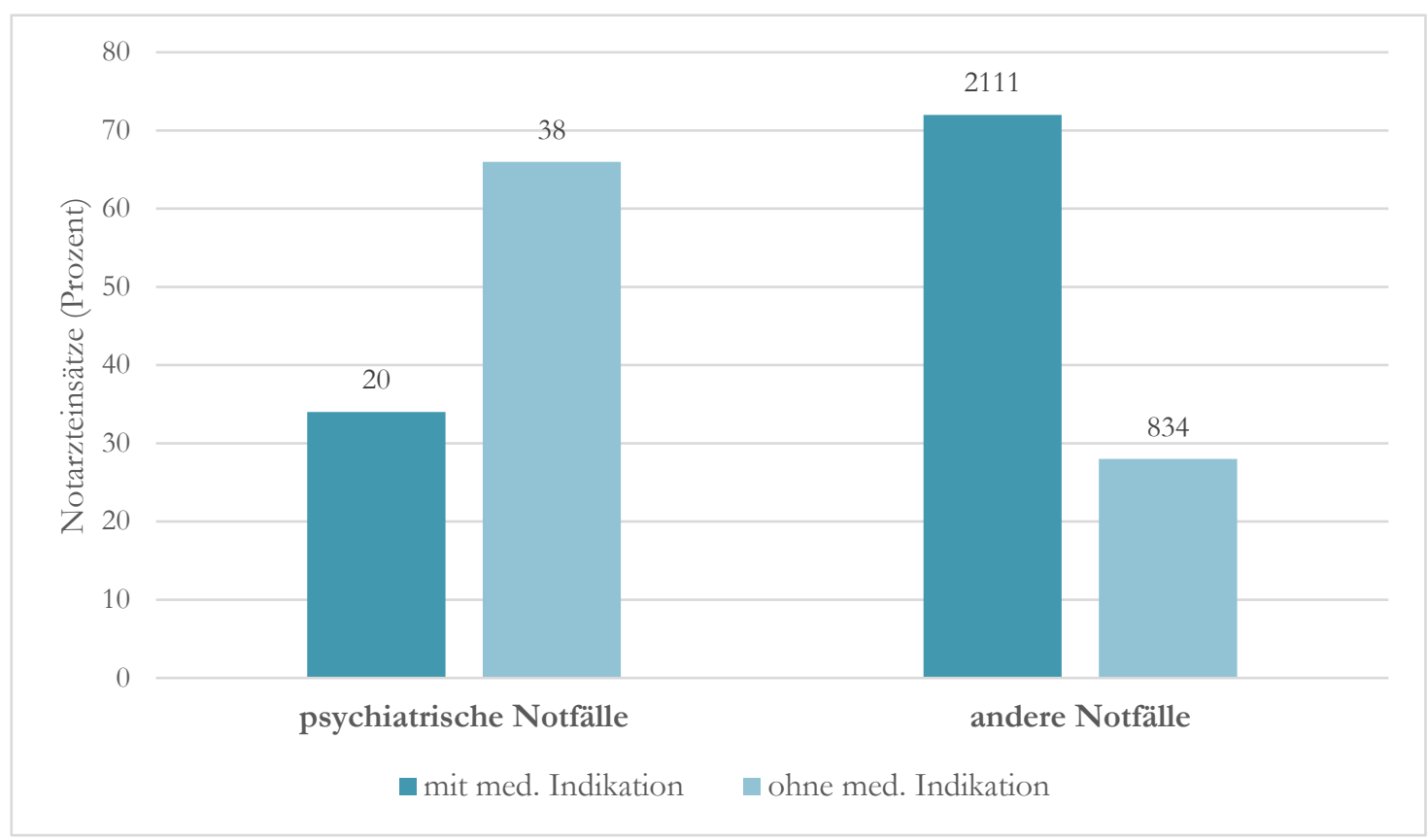

Abbildung 18: Prozentuale Verteilung der medizinischen Indikation bei psychiatrischen Notfällen vs. anderer Einsatzmeldungen. Oberhalb der Balken befinden sich die entsprechenden absoluten Fallzahlen.

\subsection{Anteil medizinisch indizierter Notarzteinsätze in den Gruppen pädiatrische Notfälle und nicht pädiatrische Notfälle}

In 74 Fällen (2,46\%) erfolgte eine Alarmierung zu Kindernotfällen. In diesem speziellen Patientenkollektiv lag bei 47 Einsätzen (64\%) eine begründete Notarztindikation nach den Studienkriterien vor, in 27 Fällen (36\%) lag keine medizinische Indikation für den Einsatz des Notarztes vor. Der Anteil medizinisch indizierter Einsätze war bei pädiatrischen Notfällen niedriger als bei den nicht pädiatrischen Notfällen (71\%), dieser Unterschied war statistisch signifikant $(p=0,013)$. 


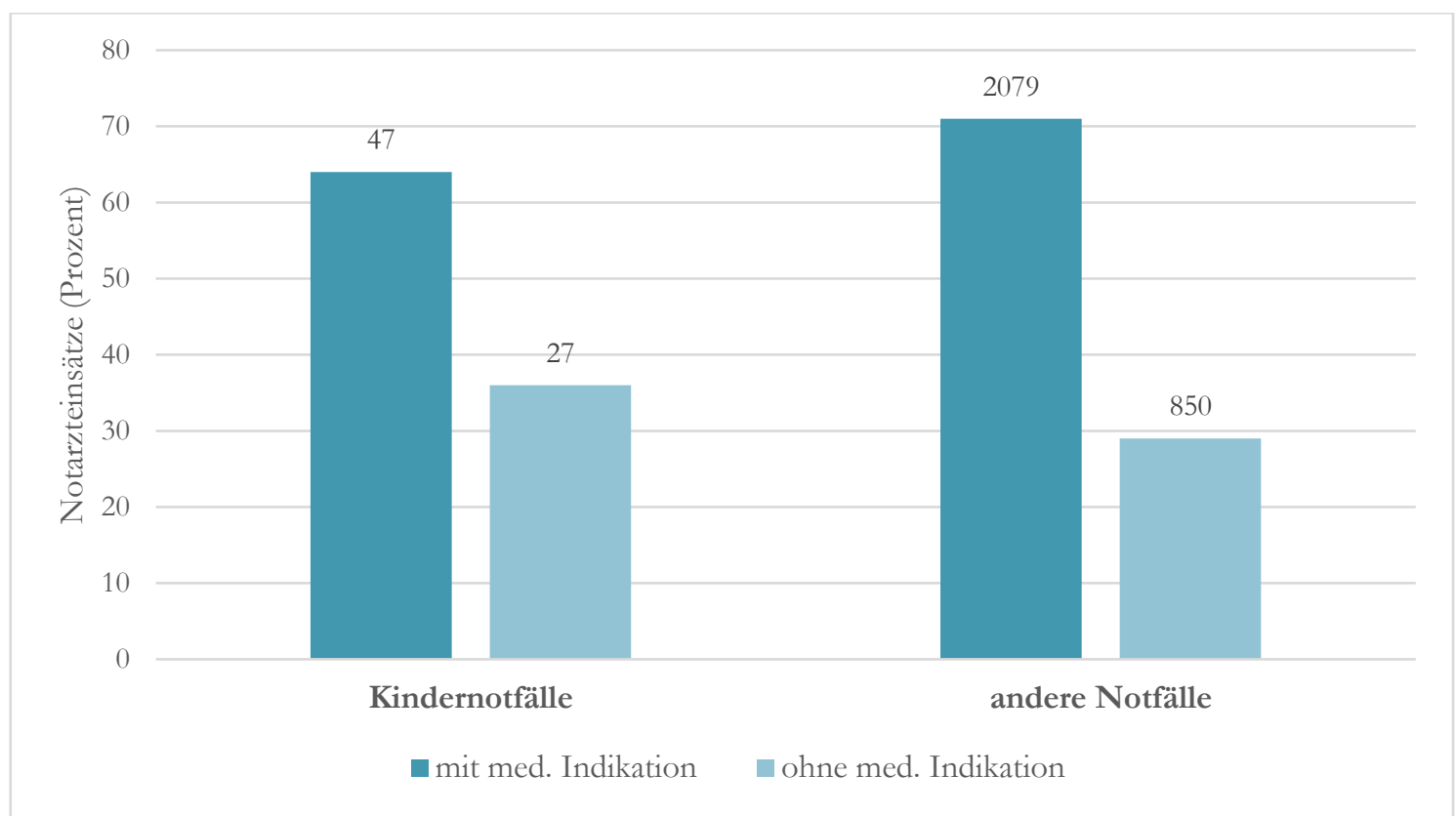

Abbildung 19: Prozentualer Anteil an medizinischer Indikation bei Kindernotfällen. Oberhalb der Balken befinden sich die entsprechenden absoluten Fallzahlen.

\subsection{NACA-Score: Häufigkeitsverteilung und Korrelation mit dem Anteil medizinisch indizierter Notarzteinsätze}

Am häufigsten (1167 Einsätze, 39\%) wurde ein NACA-Score von III vergeben, bei 69\% der Einsätze in dieser Gruppe war die Notarzt-Indikation nach unseren Kriterien gegeben.

Der zweithäufigste NACA-Score (773 Einsätze, 26\%) war IV, hiervon war in 90\% der Fälle die Indikation des Notarzteinsatzes nach den genannten Kriterien gegeben.

Die dritthäufigste Kategorie (364 Einsätze, 12\%) war NACA V, in dieser Gruppe war die Notarztindikation bei $98 \%$ der Fälle gegeben

NACA II folgt an vierter Stelle (339 Einsätze, 11\%), die Indikation des Notarzt-Einsatzes war hier in $42 \%$ der Fälle gegeben.

Die Klassifikation NACA I lag in 5\% der Einsätze vor ( $\mathrm{n}=156)$, in 14\% davon war der Einsatz des Notarztes indiziert.

NACA VI stellte mit 100 Einsätzen (3\%) den kleinsten Anteil dar, in dieser Gruppe wurden jedoch 100\% der Notarzteinsätze als medizinisch indiziert eingestuft. 
Tabelle 8: Verteilung der Einsätze nach NACA-Score.

\begin{tabular}{cccc}
\hline NACA Score & Häufigkeit & $\begin{array}{c}\text { Prozentanteil der } \\
\text { gesamten Einsätze }\end{array}$ & $\begin{array}{c}\text { mit med. } \\
\text { Indikation }\end{array}$ \\
\hline I & 156 & $5 \%$ & $14 \%$ \\
II & 339 & $11 \%$ & $42 \%$ \\
III & 1167 & $39 \%$ & $69 \%$ \\
IV & 773 & $26 \%$ & $90 \%$ \\
V & 364 & $12 \%$ & $98 \%$ \\
VI & 100 & $3 \%$ & $100 \%$ \\
\hline
\end{tabular}

Der Spearman-Korrelationskoeffizient zwischen dem Anteil medizinisch indizierter Notarzteinsätze und dem NACA-Score beträgt 0,9, es liegt somit eine statistisch signifikante Korrelation vor $(\mathrm{p}<0,005)$.

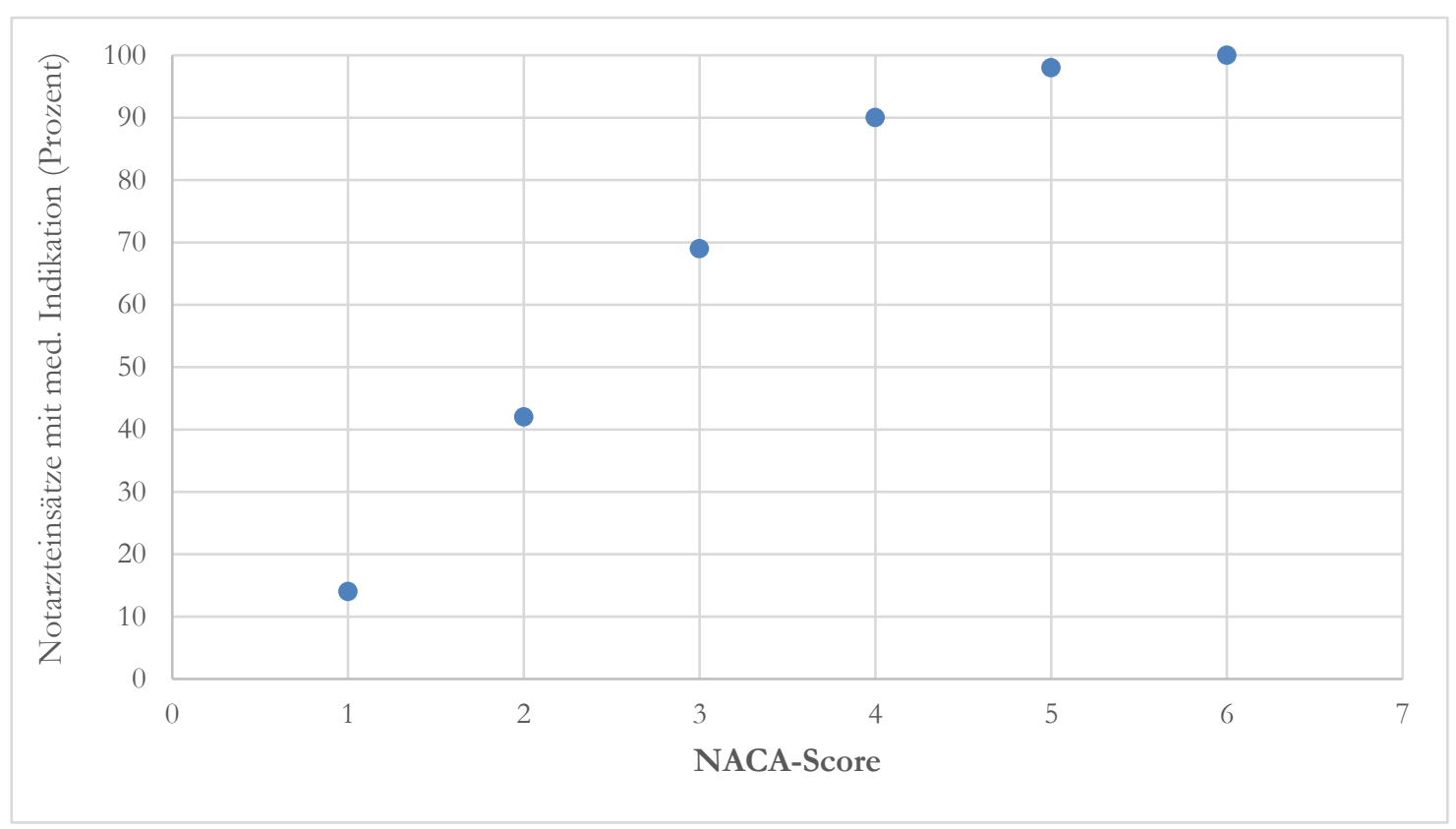

Abbildung 20: Korrelation des NACA-Scores und Einsätze mit med. Indikation (in Prozent) 


\section{Diskussion}

Die Rettung von Menschenleben bzw. die Vermeidung schwerer gesundheitlicher Schäden rechtfertigen den hohen finanziellen und personellen Aufwand, den eine Gesellschaft für einen leistungsfähigen Rettungsdienst erbringen muss. Vor dem Hintergrund dieses Aufwandes wird regelmäßig nach Optimierungsmöglichkeiten gesucht, diese Vorschläge können jedoch nur durch eine rationale Auseinandersetzung mit der untersuchten Problematik und eine konstruktive Diskussion eingeschätzt werden.

\subsection{Fragestellung, Grund für diese Untersuchung}

Im Zentrum dieser prospektiven Arbeit steht die Fragestellung, wie hoch der Anteil an Notarzteinsätzen ist, die nach einem einfachen, objektiven Schema als medizinisch indiziert eingestuft werden.

Die Bearbeitung dieser Fragestellung ist von Bedeutung, da eine aktuelle Bestandsaufnahme der Einsatzrealität arztbesetzter Rettungsmittel eine notwendige Voraussetzung ist, um eventuelle Optimierungsmaßnahmen zu diskutieren. Ein medizinisch indizierter Notarzteinsatz kann ohne Zweifel für den Patienten lebensrettend sein. Nicht gerechtfertigte Einsätze des Notarztes können jedoch mit nachteiligen Auswirkungen einhergehen.

Die Notfallversorgung der Bevölkerung steht sowohl in Niedersachsen als auch bundesweit insbesondere im ländlichen Raum vor großen Herausforderungen. Dafür liegen vielfältige Gründe vor: Auf der einen Seite nimmt die Anzahl von Rettungsdienst- und Notarzteinsätzen kontinuierlich zu; dies ist bedingt durch den demographischen Wandel (Prückner et al. 2008), durch die Schwächung sozialer Strukturen (Engel et al. 2011) und Patientengewohnheiten (Schmitt et al. 2002). Auf der anderen Seite ist sowohl eine bundesweite Ausdünnung der Krankenhausdichte (Fleßa 2005) als auch ein Rückgang der hausärztlichen ambulanten Versorgung zu beobachten. Es existiert in den letzten Jahren eine deutlich zunehmende Tendenz, dass Patienten die Notaufnahmen von Krankenhäusern über die Inanspruchnahme des Rettungsdienstes aufsuchen (Osterloh 2019).

Neue wissenschaftliche Erkenntnisse haben Diskussionen ins Leben gerufen, die fachlichen Anforderungen und strukturellen Gegebenheiten der hochqualitativen präklinischen Notfallversorgung in Deutschland anzupassen (Fischer et al. 2016). Koch et al. fordern die Suche nach Alternativen um den Rettungsdienst zu entlasten, um die Ressource „Notarzt“ für Einsätze mit akuter Vitalgefährdung freizuhalten (Koch et al. 2008).

Der Notarzt stellt im Rettungsdienst die höchst qualifizierte Instanz dar. Für die gesamte Stadt Göttingen und Umgebung stehen jedoch regelmäßig maximal zwei Notärzte zur Verfügung. Die durchschnittliche Dauer eines Notarzteinsatzes betrug in Göttingen ca. 50 Minuten (Bedarfsplan Rettungsdienst der Stadt Göttingen 2015 - Feuerwehr Göttingen 2017), gemessen von der Alarmierung bis zu dem Zeitpunkt, an dem sich der Notarzt bei 
der Leitstelle wieder freimelden kann und erneut einsatzbereit ist. Wird ein Notarzt für einen Einsatz ohne zwingende medizinische Indikation herangezogen, ist er in diesem Zeitfenster nur eingeschränkt für andere Patienten in potenzieller Lebensgefahr verfügbar. Schuster et al. beschreiben in einer Untersuchung im Gebiet Hamburg eine ähnliche durchschnittliche Einsatzdauer von 40 Minuten pro Einsatz. Eine genauere Betrachtung dieser Durchschnittswerte ist allerdings gerechtfertigt, da die tatsächliche Dauer der Einsätze zwischen 25 - 30 Minuten bei geringfügigen Krankheitsbildern und bis zu eineinhalb Stunden für schwerstkrankte Patienten betragen kann. Aus diesen Zahlen folgt eines der Argumente für den restriktiven Einsatz des Notarztes, da der Notarzt nach der Alarmierung selbst für nicht indizierte Einsätze durchschnittlich für etwa eine halbe Stunde gebunden ist (Schuster et al. 2010). Dieser Zeitbedarf basiert darauf, dass ein begonnener Patientenkontakt adäquat beendet werden muss, und auch in diesen Fällen die Pflicht zu einer gründlichen Dokumentation besteht.

Vor dem Hintergrund knapper ärztlicher Personalressourcen, die besonders in ländlichen Gebieten mit längeren Anfahrzeiten zum Tragen kommt (Luiz et al. 2011) sowie einer immer höheren Arbeitsauslastung für Mediziner, werden derzeit intensive Bemühungen unternommen, nicht ärztliche Aufgaben konsequent und effektiv zu minimieren, um Zeit für originär ärztliche Aufgaben am Patienten zurückzugewinnen.

Es zählt zu den typischen Rahmenbedingungen der präklinischen Notfallmedizin, dass der Notarzt an der Einsatzstelle häufig mit besonderen und nicht selten auch unerwarteten Situationen konfrontiert wird. Die Einschätzung von Gefahrenlagen und das Arbeiten mit deutlich eingeschränkten diagnostischen Möglichkeiten stellen eine Herausforderung sowohl für den jungen als auch für den erfahrenen Mediziner dar. Bei der Polytraumaversorgung müssen nicht nur erweiterte lebensrettende Maßnahmen (Sefrin 2012), sondern auch die komplexe technische Rettung und die Zusammenarbeit mit anderen Einsatzkräften wie Feuerwehr und Polizei beherrscht werden. Es ist essenziell, dass notärztliches Personal mit diesen Fertigkeiten vertraut ist (Kill 2008). Für eine typische notärztliche Maßnahme wie die endotracheale Intubation wurde ermittelt, dass zur sicheren Beherrschung ein Erfahrungshorizont von 60 - 80 Anwendungen erforderlich ist (Konrad 1998 und Buis et al. 2016). Eine Auswertung von ca. 100.000 Notarzteinsätzen ergab, dass bei einer Einsatzhäufigkeit von 16 Einsätzen pro Monat lediglich alle 3,7 Monate eine nicht reanimationsbedingte Intubation vorgenommen wird, und die Versorgung eines polytraumatisierten Patienten sogar nur alle 14,5 Monate erfolgt (Gries et al. 2005). Diese Zahlen verdeutlichen exemplarisch, dass das derzeitige Einsatzspektrum selbst bei regelmäßigem Einsatz im Notarztdienst nicht dazu geeignet ist, die nötige Routine für die Behandlung anspruchsvoller Notfälle zu vermitteln, da der einzelne Notarzt nur noch selten mit komplexen Notfallsituationen konfrontiert wird (Prause et al. 2007). Je mehr Notärzte notwendig sind um die stetig steigende Anzahl an Einsätzen abzudecken, umso geringer wird die Wahrscheinlichkeit, dass der einzelne Notarzt mit entsprechenden Situationen konfrontiert wird. An Standorten mit geringer Einsatzfrequenz kann daher die erforderliche 
Routine für die Beherrschung schwieriger Notfallsituationen nicht alleine durch die ausschließliche Teilnahme am Notarztdienst erworben bzw. aufrechterhalten werden (Armbruster et al. 2014).

An vielen Standorten werden Notärzte aus dem Klinikbetrieb heraus eingesetzt, d. h. müssen neben ihrer Arbeit im Rettungsdienst auch Patienten auf Stationen oder in der Notaufnahme versorgen. (Staufer 2018).Eine spezifischere Indikationsstellung für Notarzteinsätze könnten dazu beitragen, die Qualität der stationären Patientenversorgung an Kliniken zu verbessern, an denen Stationsarbeit und Rettungsdienst zeitlich miteinander konkurrieren.

Darüber hinaus ist das Unfallrisiko für die Besatzung eines Notarzteinsatzfahrzeuges ein weiteres Argument für eine sorgfältige Überprüfung der Indikation von Notarzteinsätzen. Fahrten zum Einsatzort unter Inanspruchnahme von Sondersignalen und Wegerechten erfolgen regelmäßig mit höheren Geschwindigkeiten, aktuelle Untersuchung zeigen ein bis zu fünffach erhöhtes Unfallrisiko für Fahrten unter Sondersignalen (Watanabe et al. 2019). Der Einsatz von Rettungshubschraubern ist ebenfalls mit einem erheblichen Gefahrenpotenzial verbunden (Ruskin 2019). Das Risiko Einsatz-assoziierter Todesfälle liegt hierbei noch höher als in Risikoberufen wie z. B. kommerzielle Fischerei, Holzfäller- oder Stahlarbeiten. Diese Zahlen untermauern die Forderung, Fahrten mit Sondersignalen und Rettungsflüge auf ein Minimum zu begrenzen (Tanaka und Lorenzo 2019).

Die in dieser Untersuchung gewonnenen Erkenntnisse spiegeln den derzeitigen Ist-Zustand wider und können somit die Grundlage für Diskussionen über Organisation und Ressourcenmanagement des arztbesetzten Rettungsdienstes sein.

\subsection{Studiendesign}

\subsubsection{Bewertungssystem der Notarztindikationen}

Zentrales Instrument der vorliegenden Studie ist ein einfaches System zur Beurteilung der Indikation für den Einsatz eines Notarztes. Bei der Planung der Studie stand im Vordergrund, dass die benötigten Daten einfach zu erheben sind (d. h. möglichst geringer Aufwand für die aktiven Notärzte) und objektiv interpretiert werden können.

Das erste Kriterium war die ärztliche Transportbegleitung in die Klinik, da diese Aufgabe naturgemäß nur vom Notarzt übernommen werden. Alle Einsätze, bei denen der Notarzt den Patienten aus medizinischen Gründen in die Klinik begleitet hat, wurden folglich als "indiziert" eingestuft. Für diese Informationen wurden die Notärzte nach jedem Einsatz befragt ob sie den Transport begleitet hatten, und wenn ja, ob die Begleitung aus rein medizinischen Gründen erfolgte. Letzteres unterlag der subjektiven Beurteilung des Notarztes, zu beiden Fragen lagen aber eindeutige "ja/nein"-Angaben vor.

Das zweite Kriterium, bei dessen Vorliegen der Notarzteinsatz als "indiziert" eingestuft wurde, war die Gabe von Medikamenten während des Einsatzes. Zum Zeitpunkt der 
Datenerhebung hatten erst wenige nicht ärztliche Mitarbeiter des Rettungsdienstes die Qualifikation des Notfallsanitäters erworben, zudem waren die Behandlungsalgorithmen, die die Gaben von Medikamenten durch Notfallsanitäter im Rahmen der Regelkompetenz ermöglichen, noch nicht durch den ärztlichen Leiter Rettungsdienst freigegeben. Somit war die Medikamentengabe zum Zeitpunkt der Untersuchung ebenfalls eine originär ärztliche Maßnahme. Ob Medikamente verabreicht wurden oder nicht, wurde dem elektronischen Notarztprotokoll entnommen.

Die Vorteile dieses Bewertungssystems bestehen zum einen darin, dass die Daten einfach zu erheben und objektiv zu interpretieren sind (ausschließlich "ja/nein"-Angaben). Zum anderen erfasst dieses System medizinisch gerechtfertigte Notarzteinsätze mit einer sehr hohen Sensitivität, da die Kriterien "Medikamentengabe" und "Transportbegleitung" sehr niederschwellige Indikatoren für die Notwendigkeit eines Notarzteinsatzes sind.

In dem hypothetischen Fall, dass der Notarzt zwar eine vital indizierte Maßnahme ergriffen hat (z. B. die Reposition einer Trachealkanüle), aber kein Medikament gegeben wurde und auch kein Patiententransport erfolgte, würde dieser medizinisch durchaus gerechtfertigte Einsatz des Notarztes nach unserem Schema fälschlicherweise als "nicht indiziert" eingestuft werden. Um falsch-negative Ergebnisse zu vermeiden, wurden die Notarztprotokolle von allen Einsätzen, bei denen weder eine Medikamentengabe noch ein ärztlich begleiteter Transport erfolgte, nach anderen ärztlichen Maßnahmen durchsucht, aus denen sich eine anderweitige Indikation für den Notarzt ableiten könnte. In diesen Fällen würde der Einsatz des Notarztes - abweichend zum eigentlichen Schema - als indiziert eingestuft werden.

Der Hauptnachteil des Kategorisierungssystems ist eine niedrige Spezifität, die aus der niedrigschwelligen Definition der beiden Indikations-Kriterien resultiert. In einem relevanten Anteil der Einsätze wurden Medikamente gegeben, die nicht der Therapie akut lebensbedrohlicher Zustände dienten, sondern eher zur Verbesserung des Patientenwohlbefindens eingesetzt wurden, wie beispielsweise Ondansetron. Auch wenn es in diesen Fällen keinen relevanten Einfluss auf die Versorgungsqualität gehabt hätte, wenn diese Medikamente nicht gegeben worden wären, wurde der Einsatz des Notarztes definitionsgemäß als indiziert bewertet. Bei der Studienplanung wurde daher in Erwägung gezogen, nur solche Medikamente als Kriterium für "indizierte" Einsätze zu werten, die vom behandelnden Notarzt in der jeweiligen Situation als absolut unverzichtbar eingestuft wurden, beispielsweise Adrenalin und Amiodaron bei einer Reanimation, Steroide und Antihistaminika bei einer schweren Anaphylaxie etc. Die Idee einer differenzierten Bewertung der gegebenen Medikamente wurde wegen der erheblichen Subjektivität, die mit diesem Ansatz einhergeht, jedoch nicht weiterverfolgt.

Alle Ergebnisse der vorliegenden Studie sind somit vor dem Hintergrund zu betrachten, dass das Bewertungssystem für medizinisch indizierte Notarzteinsätze zwar eine hohe Sensitivität, aber eine entsprechend niedrige Spezifität aufweist. 


\subsubsection{Argumentation für eine prospektive Studie}

Mit Hilfe elektronisch archivierter Einsatzprotokolle wäre es problemlos möglich gewesen, eine sehr viel größere Anzahl an Notarzteinsätzen retrospektiv auf die beiden Kriterien Medikamentengabe und Transport ins Krankenhaus zu überprüfen. Die meisten wissenschaftlichen Arbeiten in der Notfallmedizin basieren auf der retrospektiven Auswertung von Einsatzprotokollen, wie z. B. eine Untersuchung von Bernhard, in der die Notarzteinsätze eines Zeitraumes von 20 Jahren ausgewertet wurden (Bernhard et al. 2006).

Eine prospektive Studie impliziert einen höheren Aufwand bei der Gewinnung der Rohdaten. Zur Beantwortung unserer Fragestellung war jedoch ein prospektiver Ansatz notwendig, weil eine ärztliche Transportbegleitung nicht immer aus medizinischer Indikation, sondern häufig auch aus einsatztaktischen Gründen erfolgt, weil z. B. noch das Einsatzprotokoll geschrieben werden muss oder die Notarztwache räumlich an das aufnehmende Krankenhaus angegliedert ist, wie die Feuerwache Nord und der RTHStützpunkt am Klinikum Göttingen. Aus diesem Grund wurde bei unserer Untersuchung explizit unterschieden, ob die Transportbegleitung aus medizinischen Gründen notwendig war oder aber aus sonstigen, primär nicht medizinischen Gründen erfolgte. Diese Information wäre retrospektiv nicht zu erheben gewesen.

\subsubsection{Repräsentativität dieser Untersuchung}

In einem Zeitraum von 10,5 Monaten wurden 3003 Notarzteinsätze in der Stadt und dem Landkreis Göttingen ausgewertet. Auch wenn die Anzahl der gesamten Notarzteinsätze in den letzten Jahren auf über 5000 Einsätze pro Jahr angestiegen ist (Roessler 2019b), spiegeln die erhobenen Daten einen sehr großen Anteil der Gesamt-Einsätze wider.

Göttingen liegt im Süden der Europäischen Metropol-Region Hannover-BraunschweigGöttingen-Wolfsburg. Die beiden bodengebundenen Notarzteinsatzfahrzeige versorgen überwiegend das Stadtgebiet und das angrenzende Umland mit einer Bevölkerung von etwa 180000 Einwohnern, während der am Universitätsklinikum Göttingen stationierte RTH schwerpunktmäßig außerhalb der Stadt in einem Aktionsradius von 50-70 Kilometern eingesetzt wird und für eine Bevölkerung von etwa 350000 Einwohner (Landesamt für Statistik Niedersachsen 2019) zuständig ist. Dadurch sind in der Studie sowohl Einsätze im städtischen als auch in ländlichen Gebieten abgebildet.

Innerhalb des Zeitraums dieser Untersuchung waren die arztbesetzten Rettungsmittel mit 34 verschiedenen Notärzte besetzt. Davon befanden sich sieben Notärzte in ihrer ersten Rotation im Rettungsdienst, während 27 Notärzte bereits über umfangreiche Vorerfahrungen im Rettungsdienst verfügten oder bereits die Facharztqualifikation besaßen.

Die Charakteristika des ärztlichen Rettungsdienstes sind somit konsistent und mit anderen Metropol-Regionen Deutschlands vergleichbar. Eine Studie von Luiz et al. beschreibt ähnliche Verhältnisse in Rheinland-Pfalz; dort sind - ähnlich wie in Göttingen - die 
Notarztstandorte an Kliniken angeschlossen, der Anteil an erfahrenen Notärzten bzw. Fachärzten ist vergleichbar, und die Rettungsmittel haben ähnliche Dimensionen an Einwohnern zu versorgen (Luiz et al. 2014). Auch die Arbeit von Hegenberg beschreibt vergleichbare Verhältnisse in Bayern, sowohl für den städtischen Bezirk, als auch für ländliche Gebiete (Hegenberg et al. 2019), wobei besonders auf die deutliche Zunahme der absoluten Zahl der Einsätze pro Jahr hingewiesen wird.

Auch hinsichtlich der häufigsten Einsatzmeldungen sind unsere Ergebnisse kongruent mit anderen, aktuellen Studien, z. B. einer Untersuchung von Schlechtriemen für das Saarland (Schlechtriemen et al. 2014). Diese Daten zeigen, dass Probleme des Herz-Kreislauf-Systems, respiratorische und neurologische Symptome verglichen mit der ehemals typischen Indikation des Verkehrsunfalles oder Traumas als Alarmierungsursachen für einen Notarzteinsatz überwiegen, und belegen damit die Erweiterung des Einsatzspektrums der Notärzte im Rettungsdienst. Auch bundesweit liegen nicht traumatische Notfälle inzwischen in ihrer Häufigkeit weit vor traumatologischen Notfällen, die in den Anfangsjahren des Rettungsdienstes noch den ersten Platz belegten (Sikinger et al. 2005).

\subsection{Ergebnisse}

\subsubsection{Verteilung der medizinischen Indikation der Notarzteinsätze}

In der Bundesrepublik Deutschland finden pro Jahr rund 4,6 Mio. Rettungsdiensteinsätze statt, davon ca. 2,1 Mio., also fast die Hälfte, mit Notarztbeteiligung (Behrendt et al. 2009).

Die Ergebnisse dieser Studie zeigen, dass nach unseren Kriterien der Notarzteinsatz in 71\% „medizinische indiziert“ war, hingegen in $29 \%$,nicht medizinisch indiziert“. Da in diesen Fällen keine ärztliche Maßnahme unternommen wurde, ist zumindest kritisch zu hinterfragen, ob bei diesen fast 30\% der Einsätze die Anwesenheit des Notfallmediziners notwendig und berechtigt war.

Im Fall eines relevanten medizinischen Problems hätte der Notarzt den Patienten medikamentös behandelt, eine lebensrettende ärztliche Maßnahme eingeleitet oder den Patienten unter seiner medizinischen Aufsicht zur weiteren Versorgung in ein Krankenhaus transportiert. Da bei 29\% der Einsätze keine der genannten ärztlichen Maßnahmen erfolgten, wäre eine adäquate Patientenversorgung auch durch gut ausgebildetes nicht ärztliches Personal möglich gewesen. Perspektivisch könnte die Telemedizin die Möglichkeit eines Arztkontaktes für medizinische Rückfragen bzw. zur Absicherung des nicht ärztlichen Personal ermöglichen (Brokmann et al. 2015). Eine andere, ubiquitär verfügbare Alternative wäre die Versorgung durch den Hausbesuch eines Arztes im Rahmen des kassenärztlichen Bereitschaftsdienstes (Zdrenka 2010).

Es existieren nur wenige Untersuchungen in Deutschland, die sich im weiteren Sinn mit der Frage befassen, in welchem Prozentsatz der Fälle der Einsatz eines Notarztes tatsächlich 
indiziert ist. Hervorzuheben ist eine aktuelle Studie von Felzen et al., die zwischen Dezember 2017 und Februar 2018 in Aachen durchgeführt wurde. In dieser Untersuchung zeigte sich, dass 53,6\% der Notarzteinsätze als nicht erforderlich eingeschätzt wurden (Felzen et al. 2019). Im Gegensatz zur vorliegenden Arbeit wurden bei dieser Studie zwar keine festen Kriterien zur Definition einer medizinischen Indikation festgelegt; die Einteilung in "indiziert" und "nicht indiziert" erfolgte ausschließlich anhand der Einschätzung der eingesetzten Notärzte. Es bestätigte sich jedoch der allgemeine Eindruck, dass die Notärzte im nicht unerheblichen Maß unnötig eingesetzt wurden.

Parallel dazu zeigten andere Studien in präklinischem Setting in Schweden von 2007 (Hjälte et al. 2007) und aktueller in Australien 2018 (Eastwood et al. 2018), dass bei etwa einem Drittel der Patienten weder eine präklinische Intervention noch ein Transport stattfand, obwohl ein Rettungsmittel zur Einsatzstelle entsandt wurde. Die Ergebnisse dieser Untersuchungen sind vergleichbar mit unserer Studie.

Ausgehend von 2,1 Millionen durchgeführten Notarzteinsätzen pro Jahr in Deutschland, würde eine Verringerung um 30 Prozent zu einer Reduktion von etwa 690.000 Notarzteinsätzen im Jahr entsprechen. Dies würde erhebliches Potenzial für das Ressourcenmanagement beim Rettungsdienst bieten.

Bei den sehr häufigen Alarmierungen aufgrund partieller oder vorübergehender Beeinträchtigungen des Bewusstseins, wurde trotz der hohen Sensitivität unseres Beurteilungssystems etwa die Hälfte der Notarztalarmierungen als nicht indiziert eingestuft. Dies betrifft Alarmierungsmeldungen wie "Krampfanfall", "Schlaganfall" oder „,nicht ansprechbare Person“. Klingner et al warfen die Frage auf, ob der Notarzt bei solchen Notfällen tatsächlich notwendig ist. Ihre Ergebnisse zeigten auf einer Seite eine signifikante Verkürzung der Zeit bis zur Lyse bei Patienten mit der Verdachtsdiagnose Schlaganfall mit der Beteiligung eines Notarztes beim rettungsdienstlichen Einsatz (Klingner et al. 2017). Man könnte argumentieren, dass der Notarzt in diesen Fällen, auch bei unkompliziertem Ablauf und ohne Notwendigkeit der Einleitung weiterer Maßnahmen an der Einsatzstelle, zumindest bei dem Transport und der Auswahl eines geeigneten Krankenhauses sowie bei der Kommunikation mit den weiterversorgenden ärztlichen Kollegen eine wichtige Rolle spielt. Fairerweise muss auf der anderen Seite erwähnt werden, dass es keinen Unterschied in der Korrektheit der Diagnosestellung Schlaganfall, sowie in der gesamten Zeit der präklinischen Versorgung zwischen nichtärztlichem Personal und der Notärzte in der Studie von Klingner et al. zu beachten war, wie König et al. als Hinweis vorbringen (König et al. 2018). Eine Optimierung der Abläufe und der Kommunikation in solchen Notfällen zwischen nicht ärztlichem Personal und dem weiterbehandelten Zentrum könnte diese Vorteile für die Notarztbeteiligung verringern (Klingner et al 2018). Die Debatte bleibt somit zu diesem Thema noch offen.

Im Gegensatz zu Meldungen mit neurologischen Inhalt waren bei Alarmierungen wie ,akute Atemnot" (nach einer Untersuchung von Greiner et al. die häufigste Ursache für eine 
Krankenhausaufnahme (Greiner et al. 2018)) oder „akutes Koronarsyndrom“ in der überwiegenden Mehrzahl der Fälle Maßnahmen des Notarztes an der Einsatzstelle erforderlich. Savage et al. und Lebiedz et al. konnten in ihren Untersuchungen zeigen, dass die korrekte und zügige Diagnosestellung durch den Notarzt zur Reduktion der Zeit bis zum Erreichen des Herzkatheterlabors führt (Savage et al. 2014) und damit zur Verbesserung des Behandlungsergebnisses beiträgt (Lebiedz et al. 2011). Auch andere Autoren weisen auf positive Effekte durch das Heranziehens des Notarztes bei herzischämischen Ereignissen hin (Takeuchi et al. 2015). In der Zukunft könnten neue, diagnostische Verfahren wie die präklinische Bestimmung von Herzenzymen an Bedeutung gewinnen. Diese könnten die Genauigkeit präklinisch gestellter Diagnosen und die Identifikation von kritischen Notfällen verbessern und somit dazu beitragen, insbesondere die Indikation zum ärztlich begleiteten Transport enger zu stellen (Stengaard et al. 2016).

\subsubsection{Unterschiede zwischen RTH und NEF}

Obwohl aus medizinischer Sicht für den Einsatz von RTH und NEF der gleiche Indikationskatalog gilt (Schellhaaß und Popp 2014), scheint in der Realität die Alarmierungsschwelle für ein Notarzteinsatzfahrzeug niedriger zu sein als für einen Rettungshubschrauber. Dies geht aus unseren Ergebnissen hervor, die in der Untergruppe der NEF-Einsätze einen signifikant niedrigeren Anteil medizinisch indizierter Einsätze als in der Untergruppe der RTH-Einsätze zeigten. Ein restriktiverer Einsatz des Rettungshubschraubers verglichen mit dem NEF könnte darin begründet sein, dass sich der Rettungshubschraubers hinsichtlich der Einsatzkosten und des Unfallrisikos deutlich von bodengebundenen Rettungsmitteln unterscheidet (Hinkelbein et al. 2008). Der Rettungshubschrauber wird vor allem dann eingesetzt, wenn er deutlich früher am Notfallort eintreffen kann als ein bodengebundenes Rettungsmittel (Gries et al. 2014).

Traditionell wurden Rettungshubschraubers größtenteils bei Verkehrsunfällen mit Personenschaden eingesetzt, aber auch heute noch stellen Traumata (bis zu 40\% der Luftrettungseinsätze) (Schweigkofler et al. 2015) und sehr schwer erkrankte Patienten einen großen Teil der Einsätze dar. In diesen Fällen scheinen die Patienten vom Einsatz der Luftrettung besonders zu profitieren (Rezar et al. 2017).

Der Einsatz eines Rettungshubschraubers bringt mehrere Vorteile mit sich. Zum einen sind die dort eingesetzten Notfallmediziner in der Regel Fachärzte mit einer langjährigen Routine in der Luftrettung (Hofer und Voelckel 2014), und verfügen daher über ein hohes Maß an Kompetenz (Andruszkow et al. 2012).

Ein weiterer Vorteil ist die Verkürzung der Eintreffzeiten sowie der deutlich schnellere Transport in das versorgende Krankenhaus. Dieser Vorteil kommt insbesondere bei größeren Entfernungen und bei hohem Verkehrsaufkommen (nicht nur in Städten) zum Tragen, und spiegelt sich was sich in diversen Studien eindeutig in Form eines besseren Outcomes und Überlebensraten bei zeitkritischen Erkrankungen wider. Wie die 
Studiengruppen von Gries et al. sowie Schweigkofler et al. belegten, profitieren insbesondere Patienten mit stumpfen Traumata und polytraumatisierte Patienten von der Versorgung durch einen RTH (Gries et al. 2008 und Andruszkow et al. 2016) Zu vergleichbaren Ergebnisse kam auch eine französische Arbeitsgruppe von Desmettre et al. (Desmettre et al. 2012). Darüber hinaus konnte im skandinavischen Raum gezeigt werden, dass der Einsatz des RTH beim akuten Koronarsyndrom (engl. ACS) mit verkürzten Zeiten bis zur perkutanen Koronarintervention (engl. PCI) assoziiert ist (Knudsen et al. 2012 und Hesselfeldt et al. 2013). Zudem konnten Studien aus Österreich zeigen, dass auch bei neurologisch-ischämischen Krankheitsbildern der Einsatz der Luftrettung zu Zeitvorteilen und höheren Lyse-Raten führt (Reiner-Deitemyer et al. 2011).

Insbesondere für den Transport in ein spezielles, individuell geeignetes Zentrum zur weiteren Therapie ist der Rettungshubschrauber den bodengebundenen Rettungsmitteln überlegen (Andruszkow et al. 2013). Dies gilt auch im internationalem Kontext (Tsuchiya et al. 2016). Die frühzeitige Triage und schnellstmögliche Verlegung in das am besten geeignete Zentrum erklärt den Zusammenhang zwischen dem Einsatz des RTH und den besseren Behandlungsergebnissen (Fjaeldstad et al. 2013) und ist von großem Stellenwert insbesondere bei schwerkranken Kindern (Brown et al. 2016). Alle diese Faktoren spiegeln sich in besseren Überlebensraten und einer geringeren Ausprägung bleibender Schäden der Patienten wider (Stewart et al. 2019).

Im Falle limitierter Ressourcen stellt sich vermehrt die Frage, wann ein Rettungshubschrauber zum Einsatz kommen soll (Laatz et al. 2019). In afrikanischen Ländern erfolgt der Einsatz des Hubschraubers hauptsächlich aufgrund klinischer Parameter und der geographischen Lage, anstatt anhand von Indikationskatalogen. Die Verfügbarkeit eines Rettungshubschraubers hat sogar eine Auswirkung auf die Lebenserwartungen in ländlichen Gebieten (Zakariassen et al. 2019), in denen Versorgungslücken bei großen Entfernungen und knappen Ressourcen teilweise durch die Luftrettung überbrückt werden.

Bei allen genannten Vorteilen, die mit dem Einsatz von Rettungshubschraubern verbunden sind, muss auf Einschränkungen der Einsetzbarkeit bei unzureichenden Lichtverhältnissen (Aschenbrenner et al. 2015) und schlechten Wetterbedingungen (Klier et al. 2015) hingewiesen werden. Das flächendeckende Netz der Luftrettung stellt daher lediglich eine Ergänzung des bodengebundenen Notarztsystems dar, auch wenn derzeit Bestrebungen existieren, einen regulären Nachtbetrieb zu etablieren.

\subsubsection{Verhältnisse bei primärer Alarmierung vs. Nachalarmierung}

Bei gut einem Fünftel aller Einsätze dieser Untersuchung (21\%) wurde der Notarzt nicht primär aufgrund der Einsatzmeldung alarmiert, sondern wurde von Einsatzkräften (i. d. R. RTW-Besatzungen) nach deren Eintreffen beim Patienten nachgefordert.

Die Ergebnisse dieser Studie zeigen bei Nachalarmierungen einen signifikant höheren Anteil an medizinisch indizierten Notarzteinsätzen als bei primärer Mitalarmierung durch den 
Disponenten der Leitstelle. Das heißt, wenn ein Rettungs- oder Notfallsanitäter, eine Pflegekraft oder ein Mediziner (jeglicher Fachrichtung) anhand seiner fachlichen Einschätzung vor Ort entscheidet den Notarzt nachzufordern, liegt eine medizinische Indikation für den Notarzteinsatz zu einem signifikant höheren Anteil vor als bei Primäralarmierungen durch die Rettungsleitstelle.

Der Mangel an der Ressource „Notarzt“ könnte dadurch ausgeglichen werden, dass notfallmedizinische Standardmaßnahmen zukünftig häufiger und regelhaft von qualifiziertem Rettungsfachpersonal übernommen werden, und Notärzte deutlich gezielter eingesetzt werden (Gretenkort et al. 2016). Sollte sich aus dem Meldebild nicht eindeutig eine Notarztindikation ergeben, könnte im Zweifelsfall zunächst ein RTW entsendet werden. Im Falle einer vital bedrohlichen Situation könnte der Notarzt umgehend nachgefordert und die Zeit bis zu seinem Eintreffen für Maßnahmen genutzt werden, die für die ärztliche Therapie ohnehin erforderlich sind (z. B. Anbringen des Monitorings, Legen von i.v.-Zugängen, Erheben einer genauen Anamnese etc.).

Durch die Einführung des Notfallsanitäters wurde die medizinische Ausbildung von nicht ärztlichem Rettungsdienstpersonal bundesweit standarisiert und verbessert (Lechleuthner und Neupert 2015), sodass in vielen Fällen eine adäquate Versorgung von Notfallpatienten auch ohne Notarzt möglich ist. In Verbindung mit unseren Ergebnissen kann geschlussfolgert werden, dass es insbesondere im Fall einer geringen Verfügbarkeit von Notärzten sinnvoll sein kann, im Zweifelsfall zunächst einen RTW zu alarmieren. Dieser kann bei nicht vital bedrohlichen Notfällen die vollständige Versorgung übernehmen, und in allen anderen Fällen nicht nur eine "Filterfunktion" für den Einsatz des Notarztes übernehmen, sondern auch bis zu dessen Eintreffen bereits erste therapeutische Maßnahmen einleiten.

Im Nachbarland Österreich existiert ebenfalls ein zweistufiges Rettungssystem für die Notfallversorgung, vergleichbar zu dem deutschen System. Nicht ärztliches Personal wird primär bei nicht lebensbedrohlichen Notfällen eingesetzt und der Notarzt nur dann entsandt, wenn Hinweise auf einen vital bedrohlichen Notfall bestehen. In der Stadt Graz wurde dieses System vor dreißig Jahren um eine dritte Stufe erweitert: Zu Notfällen, in denen eine akute Lebensgefahr nicht sicher ausgeschlossen werden kann, können in Notfall- und Rettungsmedizin ausgebildete Medizinstudenten (die sogenannten Medizinercorps) entsendet werden. Diese Medizinercorps verfügen über einen ähnlichen Ausbildungsstand wie deutsche Notfallsanitäter und können ebenfalls den Notarzt zur Einsatzstelle nachfordern, wenn nach ihrer Ersteinschätzung eine vital bedrohliche Situation vorliegt (Prause et al. 2017).

Nachteilig ist an dieser Vorgehensweise, dass ein Zeitverlust bis zum Eintreffen des Notarztes entsteht (Mürdel 2015). In Deutschland ist die Dichte der RTW-Standorte deutlich höherer als die der Notarztstandorte. Die durchschnittliche Eintreffzeit eines RTW liegt bei 8,4 Minuten, während sie für den Notarzt 14 Minuten beträgt (Schmiedel und Behrendt 
2015). Wird der Notarzt durch die RTW-Besatzung nachalarmiert, verzögert sich sein Eintreffen gegenüber einer Primäralarmierung mindestens um die Anfahrtszeit des RTW. Aufgrund der längeren Anfahrtswege wäre diese Zeitverzögerung in ländlichen Gebieten deutlicher ausgeprägt als in städtischen Regionen. Das RTW-Team kann jedoch unverzüglich mit der eigenverantwortlichen medizinischen Erstversorgung beginnen, bis der nachgeforderte Notarzt eintrifft (Lohs 2018). Aufgrund ihrer hohen Fachkompetenz können insbesondere Notfallsanitäter auch bei akut lebensbedrohlichen Zuständen die Zeit bis zum Eintreffen des Notarztes mit effektiven Therapiemaßnahmen überbrücken (z. B. mit Herzdruckmassage und Beatmung bei einem Herz-Kreislauf-Stillstand oder mit der Anlage eines Druckverbandes bei einer externen Blutung), so dass es kein therapiefreies Intervall entsteht. Zudem können Vorbereitungen für ärztliche Maßnahmen getroffen werden (z. B. Aufziehen von Medikamenten die Vorbereitung einer endotrachealen Intubation), sodass diese Maßnahmen sofort nach dem Eitreffen des Notarztes ohne weitere Zeitverzögerungen durchgeführt werden können. Verschiedene Untersuchungen konnten zeigen, dass eine prompte Notfallversorgung (Chen et al. 2009) und eine frühzeitige Krankenhausaufnahme mit korrekter Übergabe zur weiteren Behandlung (Gupta et al. 2017 und Touray et al. 2018) für das Überleben in einem Notfall maßgeblich sind. Das Rettungsdienstpersonal muss aufgrund seiner Qualifikation in der Lage sein, längere Anfahrtszeiten des Notarztes zu überbrücken, ohne dass Nachteile für den Patienten entstehen.

Das Rendezvous-System erweist sich in dieser Hinsicht ebenso sinnvoll wie der sekundäre, gezielte Einsatz des Notarztes bei Nachforderung durch einen primär eintreffenden RTW.

\subsubsection{Unterschiede nach Erreichbarkeit des Hausarztes}

Notaufnahmen von Kliniken werden von einem Großteil der Patienten außerhalb der regulären Regelarbeitszeiten aufgesucht, d. h. in Abendstunden oder am Wochenende (Gries et al. 2013). Auch der Rettungsdienst wird mit zunehmender Häufigkeit außerhalb der Hausarztsprechstunden gerufen, wie Rau et al. nach einer Untersuchung in NordrheinWestfalen berichten (Rau et al. 2006). Eine Verlagerung der hausärztlichen Versorgung auf den Notarzt könnte zur Folge haben, dass der Anteil medizinisch indizierter Notarzteinsätze außerhalb der regulären Praxisöffnungszeiten niedriger ist als in Zeiträumen, in denen eine hausärztliche Versorgung gegeben ist.

Die Ergebnisse dieser Studie zeigen, dass zwar mehr Notarzteinsätze außerhalb der Öffnungszeiten von Hausarztpraxen stattfinden. Der Anteil medizinisch indizierter Notarzteinsätze weist jedoch während und außerhalb der regulären Praxisöffnungszeiten keinen signifikanten Unterschied auf.

Vermutlich suchen viele Patienten den Zugang zum Gesundheitssystem über die Einweisung durch einen Notarzt oder durch direktes Aufsuchen der Notaufnahme (Schmiedhofer et al. 2017). Ebenso ist einigen Menschen der Zugang zur medizinischen 
Versorgung im niedergelassenen Bereich erschwert, z. B. ist eine Hausarztbindung zumindest in Großstädten nicht immer gegeben (Luiz 2013).

Churpek et al. konnten zeigen, dass der Rettungsdienst während der Nacht deutlich seltener alarmiert wird, woraus schlechtere Ergebnisse hinsichtlich der Überlebensraten bei schweren kardiovaskulären Problemen resultieren (Churpek et al. 2017). Die Arbeit von Hegenberg et al. zeigt zudem einen höheren Schweregrad der Erkrankung von Patienten, die abends oder nachts durch einen Notarzt in eine Notaufnahme eingeliefert werden (Hegenberg et al. 2019). Dieses Phänomen konnte in unserer Studie allerdings nicht beobachtet werden.

\subsubsection{Erfahrungshorizont der Notärzte}

Die Auswertung unserer Daten konnte keinen Zusammenhang zwischen dem Erfahrungshorizont der eingesetzten Notärzte und dem Anteil als medizinisch indiziert bewerteter Einsätze zeigen.

Notärzte mit Vorerfahrung im Rettungsdienst beließen in gleichem Ausmaß Patienten nach einer ambulanten Behandlung an der Einsatzstelle (i. d. R. zu Hause) als diejenigen Notärzte, die ihre erste Rotation im Rettungsdienst absolvierten. Dies scheint vorangegangenen Untersuchungen zu widersprechen. Die Arbeit von Ocker et al. zeigte, dass erfahrene Notärzte es sich häufiger zutrauten Patienten ambulant zu behandeln, um die Notaufnahmen der Kliniken zu entlasten (Ocker et al. 2006).

Darüber hinaus begleiteten Notärzte, die ihre erste Rettungsdienst-Rotation absolvierten, ihre Patienten häufiger aus medizinischen Gründen in die Klinik als Notärzte mit Vorerfahrung im Rettungsdienst. Es ist nicht anzunehmen, dass die Schweregrade der Erkrankungen in diesen beiden Gruppen signifikant unterschiedlich waren. Es scheint daher wahrscheinlicher zu sein, dass Notärzte mit einer geringeren Erfahrung im Rettungsdienst Situationen kritischer einschätzen als erfahrenere Kollegen, und Patienten daher häufiger begleiten (Knapp et al. 2008). Ein Notarzt kann sich als abkömmlich für den nächsten Einsatz bei der Leitstelle melden, wenn die Transportbegleitung in die Klinik nicht aus medizinischer Sicht erforderlich ist. Dadurch sind weniger erfahrene Ärzte häufiger bis zur Übergabe des Patienten im Krankenhaus an den Einsatz gebunden.

\subsubsection{Bedeutung der Altersverteilung}

Die Zahlen unserer Untersuchung ergeben, dass 70\% der Patienten, die den Rettungsdienst in Anspruch nahmen, über 50 Jahre alt waren. Ein steiler Anstieg der Einsätze ist ab einem Alter von 70 Jahren zu verzeichnen. Die Alterskategorie über 65 Jahre, deren Angehörige als „Senioren“ bezeichnet werden (Thümmler 2003), umfasste einen Anteil von 52,3\% von allen Einsätzen. Anders formuliert war jeder zweite Patient über 65 Jahre alt. Dies korreliert mit dem beschriebenen Bevölkerungswandel im Sinne einer „Alterung“ der Gesellschaft deutschlandweit (Statistisches Bundesamt 2019) und in Europa (Lawrence 2013). Auf Grundlage dieser Beobachtung kann davon ausgegangen werden, dass die Inanspruchnahme 
des Rettungsdienstes aufgrund des demographischen Wandels zukünftig weiter zunehmen wird.

In der Altersgruppe ab 65 Jahren lag der Anteil medizinisch indizierter Notarzteinsätze mit 74,65\% höher als bei Patienten unter 65 Jahren (66,92\%). In dieser Altersklasse trifft der Rettungsdienst oft auf multipel vorerkrankte Patienten, für deren komplexe Krankheitsbilder die Behandlungsalgorithmen des Rettungsdienstes häufig nicht anwendbar sind. Die Multimorbidität und die begleitende Polypharmakotherapie in diesen Patientenkollektiv wurde in einer Untersuchung von Wutti et al. belegt (Wutti 2014), darüber hinaus belegen Daten aus den Vereinigten Staaten, dass jeder sechste Patient über 65 Jahre innerhalb von dreißig Tagen nach einer Entlassung erneut vom Rettungsdienst ins Krankenhaus transportiert wird (Evans et al. 2017). Das Alter der Patienten bleibt wegen des Volumens der Einsätze und ihr Komplexität für den Rettungsdienst sehr relevant.

Immer häufiger wird der Notarzt auch zu vereinsamten Senioren gerufen. Besonders in Alten- und Pflegeheimen rufen viele ältere Patienten bzw. ihre Betreuer in ihrer Hilflosigkeit häufig zuerst den Rettungsdienst (Aruga 2013), anstatt andere Instanzen zu kontaktieren. Es muss daher angenommen werden, dass der Rettungsdienst oftmals Versorgungslücken schließen müssen (Kosan 2012). Carron et al. konnten in der Schweiz das Ausmaß dieses Phänomens belegen: Im Zeitraum von 2004 bis 2013 nahm im Schweizer Kanton Waadt bei Bewohnern von Pflegeheimen mit einem Alter von über 65 Jahren die Anforderung des Notarztes um 200\% zu (Carron et al. 2015). Ähnliche Verhältnisse lassen sich im letzten Dezennium auch in Deutschland und Österreich beobachten (Heppner et al. 2014).

Die zunehmende Hilfsbedürftigkeit alter, multimorbider Patienten und Pflegeheimbewohnern erfordern aufgrund des demographischen Wandels neue Versorgungskonzepte. Eine wichtige Rolle spielt eine exakte und sofort zugängliche Patientendokumentation, um im Notfall oder bei terminalen Zuständen medizinische Entscheidungen auf die individuelle Situation des Patienten abstimmen zu können. Ebenso denkbar wären Handlungsprotokolle für die häufigsten pflegerischen und, bis zum einen bestimmten Grad auch medizinischen Vorkommnisse im Pflegeheim (Schuster et al. 2016), mit denen ein Teil der nicht indizierten Einsätze für den Notarzt vermieden werden könnte.

\subsubsection{Besondere Alarmierungsmeldungen: Die Kindernotfälle, das Trauma, die psychosozialen Notfälle}

Eine gesonderte Betrachtung unserer Ergebnisse erfolgt für Kindernotfälle sowie traumatologische Notfälle. Diese Notfälle erfordern vom Notarzt wegen ihrer pathophysiologischen Komplexität und der Spezifität ihrer Behandlung ein hohes Maß an Kompetenz (Wooldridge et al. 2019 und Brown et al. 2017). Desgleichen wird wegen ihrer fachspezifischen Besonderheiten auch auf die psychiatrischen Notfälle näher eingegangen, da in der Literatur der sinnhafte Einsatz des Rettungsdienstes in diesen Fällen immer wieder in Frage gestellt wird (Karutz H. 2014). 
Das Meldungsbild "Kindernotfall" ist im Einsatzkatalog enthalten und führt in der Regel zur Alarmierung des Notarztes. Nach unseren Ergebnissen ist diese Alarmierung nicht zwingend medizinisch begründet; in dieser Untersuchung führte der Notarzt bei über einem Drittel der Kindernotfälle weder eine ärztliche Maßnahme noch den begleiteten Transport durch.

Gleichwohl ist hervorzuheben, dass Kindernotfälle zwar selten sind, aber oft die Expertise des Rettungspersonals herausfordern (Eich et al. 2009) und deshalb oft als sehr anspruchsvoll gewertet werden (Bartels 2001). Die Behandlung von Kindern erfordert aufgrund der speziellen Charakteristika dieses Patientenkollektivs, z. B. bei der Sicherung des Atemweges (Engelhardt und Weiss 2012) oder der Anlage eines intravenösen bzw. intraossären Zugangs (Horton und Beamer 2008) eine besondere medizinisch-technische Expertise (Meißner 2017). In diesem Zusammenhang konnten mehrere Studien die Überlegenheit von Notärzten gegenüber nicht ärztlichem Rettungsdienstpersonal bei der Durchführung lebensrettender Maßnahmen zeigen, wobei frühzeitige und qualifizierte Interventionen oft zu guten Behandlungsergebnissen führten (Heinzel et al. 2011). Dies kommt insbesondere bei traumatologischen Kindernotfällen zum tragen (Schlechtriemen et al. 2006), die daher obligat als Notarztindikation beibehalten werden sollten (Sefrin 2009). Die korrekte Einschätzung der Schwere einer Erkrankung bereits in der präklinischen Phase kann signifikant zur Reduktion der Intensivpflichtigkeit und der Mortalität bei Kindern beitragen (Corfield et al. 2018).

Das Trauma ist die häufigste Todesursache bei Menschen unter 45 Jahren und stellt in allen Altersklassen in den westlichen Industrienationen die dritthäufigste Todesursache dar (Pfeifer et al. 2009). Trotz Abnahme der absoluten Notfälle aufgrund der Fortschritte in der Fahrzeugtechnik, der Optimierung der Sicherheit von Verkehrswegen, der Verfeinerung der Gesetzgebung sowie allgemeiner und spezifischer Präventionsprogramme in der Industrie und im häuslichen Umfeld, sind die Zahlen der Todesfälle vor dem Erreichen des Krankenhauses immer noch relevant (Kleber et al. 2012). Über Jahre hinweg waren die Unfälle mit Schwerverletzten bei weitem die Hauptindikation für den Einsatz des Notarztes. Fast zwei Drittel aller traumatologisch bedingter Todesfälle treten präklinisch ein und fallen damit in den Zuständigkeitsbereich des Rettungsdienstes. Daher war die ärztliche Versorgung des Traumas das primäre Motiv der notfallmedizinischen Versorgung, wofür ursprünglich die Strukturen des notarztbesetzten rettungsdienstlichen Systems konzipiert wurden (Buschmann et al. 2013). In den letzten Jahren haben jedoch die Verkehrsunfälle deutlich abgenommen während Notarzteinsätze bei akuten medizinischen Erkrankungen stark zugenommen haben. Der aktuelle Ausbildungsstandard sieht jedoch immer noch die Versorgung von polytraumatisierten Patienten als einen Schwerpunkt der Ausbildung für Notärzte vor (Reifferscheid et al. 2010).

Bundesweit betreffen traumatologische Notfälle abhängig vom Standort etwa zwischen 15$20 \%$ der gesamten Einsätze (Gries et al. 2003), in unserer Untersuchung lagen traumatologische Notfälle in 12\% der gesamten Einsätze vor. Eine medizinische Indikation 
für den Notarzt war nach den Kriterien dieser Untersuchung bei 82\% der Einsätze gegeben, bei lediglich 18\% der Einsätze mit traumatologischem Hintergrund erfolgte weder eine Medikamentengabe noch eine Transportbegleitung durch den Notarzt.

In dieser Untersuchung lag der Anteil an nicht traumatologischen Einsätzen bei 88\%. Von der Gesamtheit dieser nicht traumatologischen Notfälle wurde in 69\% der Fälle der Einsatz des Notarztes nach unseren Kriterien als indiziert gewertet, also in einem deutlich geringerem Prozentsatz Ausmaß als bei traumatologischen Fällen. Dieser Unterschied erwies sich ebenfalls als statistisch signifikant und bestätigt die Notwendigkeit einer notärztlichen Versorgung bei schweren Traumata. Dieser Ansatz spiegelt sich in den Algorithmen und Leitlinien für Polytraumaversorgung (Helm et al. 2018) wider, in welchen der Einsatz eines Notarztes in Hinblick auf die gesundheitliche Vorteile für Patienten (engl. patient outcomes) klar empfohlen wird (Oramary et al. 2019).

Psychiatrische Notfälle wiesen in dieser Untersuchung den niedrigsten Anteil medizinisch indizierter Notarzteinsätze auf. Insgesamt ist der Anteil psychiatrischer Notfälle in Göttingen mit 2\% relativ gering, in anderen größeren Städten und Ballungsräumen wurde der Anteil psychiatrischer Einsätze bis zu 14\% beschrieben (Sefrin und Ripberger 2008). In unserer Untersuchung lag in nur 27\% dieser Fälle eine medizinische Indikation für den Notarzt vor, demnach erfolgte in fast drei Vierteln der Fälle keine spezifisch ärztliche Maßnahme durch den Notarzt.

Seit Jahren werden daher verschiedene Diskussionen geführt, ob ein Notarzt bei psychiatrischen Notfällen erforderlich ist (Wilhelm 2003). Zwar gibt es aktuell keine genauen Zahlen über die Häufigkeit psychosozialer Probleme in der Notfallmedizin; es lässt sich aber vermuten, dass die Anzahl an psychiatrischen Fällen lange unterschätzt wurde. Nach der Einschätzung von Pajonk werden bundesweit pro Jahr etwa 500.000 Patienten mit psychiatrischen Erkrankungen präklinisch versorgt (Pajonk 2015). Ferner besteht die Vermutung, dass die Anzahl an psychosozialen Notfällen derzeit steigt. Gründe hierfür sind Veränderungen in den Strukturen der stationären und ambulanten Versorgung sowie eine Zunahme destabilisierender psychosozialer Faktoren in der Gesellschaft wie Arbeitslosigkeit, fehlende Integration und soziale Einbindung sowie die Isolation von alleinstehenden Senioren.

Der Notarzt wird immer häufiger zu vereinsamten Personen in einer labilen psychischen und unsicheren sozialeren Lage gerufen. In der letzten Zeit wird vom Notarzt vielerorts immer häufiger erwartet, in gewissen Umständen die Rolle des „psychosozialen Erlösers“ zu übernehmen (Schmitt et al. 2002). Es scheint jedoch legitim zu hinterfragen, ob der Notarzt der richtige Ansprechpartner ist, um diese schwierigen Situationen zu bewältigen oder ob diese wertvolle Ressource eingespart werden könnte, wie unsere Ergebnisse in der großen Mehrheit der Fälle zeigen. Diese Frage ergibt sich insbesondere auch aus der Tatsache, dass viele dieser Einsätze mit einem hohen Zeitaufwand einhergehen und den Notarzt dadurch längerfristig für andere Notfälle blockieren (Kirchner und Hill 2013). 
Als mögliche Lösung könnten Strukturen für eine Zusammenarbeit mit bereits vorhandenen psychosozialen Einrichtungen geschaffen werden, beispielsweise ein vereinfachter Zugang zur stationären Behandlung oder eine rund um die Uhr verfügbare ambulante psychiatrische Versorgung. Hierdurch könnte eine bessere Versorgung von Menschen in psychosozialen Problemsituationen erreicht werden, was zu einer Entlastung der Notärzte beitragen würde (Luiz 2013).

\subsubsection{Der NACA-Score für die Bewertung der Notarzteinsätze}

Der NACA-Score weist bei der Kategorisierung von Notfalleinsätzen eine Reihe von Vorteilen auf: Er wird obligat bei jedem Notfallpatienten erhoben und auf jedem Behandlungsprotokoll dokumentiert (Moecke et al. 2004). Der Score ist unabhängig von physiologischen Messwerten, er lässt sich sowohl bei Erkrankungen als auch bei Verletzungen anwenden und ist damit für die präklinische Notfallmedizin, in der nur auf eingeschränkte diagnostische Möglichkeiten zurückgegriffen werden kann, besonders geeignet (Messelken und Schlechtriemen 2003).

Durch die obligate Dokumentation des NACA-Scores in den Einsatzprotokollen können auch große Patientenkollektive sehr einfach, objektiv und gegebenenfalls sogar retrospektiv ausgewertet werden, ohne dass weitere Informationen über den Patienten erforderlich sind (Messelken et al. 2011).

Jedoch wird auch auf verschiedene Unzulänglichkeiten dieses einfachen Scoring-Systems hingewiesen. Zwar gab es in der Vergangenheit Vorhaben, die Indikation für den Notarzt direkt anhand des NACA-Scores retrospektiv zu bewerten (Messelken und Dirks 2001). Schlechtriemen et al. zweifeln jedoch an, dass sich ein NACA-Grenzwert formulieren lässt, oberhalb dessen die Indikation für einen Notarzteinsatz gegeben ist (Schlechtriemen et al. 2005b). Ein Cut-off Wert (engl. Abgrenzung der Punktzahl) des NACAs ist für die Bewertung der Notarztindikation eines Einsatzes nicht geeignet, wie an den folgenden drei exemplarischen Beispielen gezeigt wird.

Ein besonders breiter Interpretationskorridor besteht bei der Vergabe von NACA IV ("akute Lebensgefahr nicht auszuschließen"). Aufgrund der begrenzten diagnostischen Möglichkeiten und der oft nur unzureichend bekannten Anamnese im Rettungsdienst kann präklinisch ein akut lebensbedrohlicher Zustand (z. B. ein asymptomatisches großes Aortenaneurysma) genaugenommen niemals mit letzter Sicherheit ausgeschlossen werden.

Ein bewusstloser Patient mit einer schweren Hypoglykämie befindet sich objektiv in akuter Lebensgefahr und ist somit als NACA V einzustufen. Die Diagnose ist mittels elektronischer Blutzucker-Messgeräte jedoch so eindeutig zu stellen und die Therapie so standardisiert, dass dieser Zustand ohne jeden Qualitätsverlust auch von Rettungsassistenten behandelt werden kann (Alessandrini et al. 2012), insbesondere, wenn mit Einführung des Notfallsanitäters das Spektrum der Regelkompetenzen erweitert wird. Trotz des NACA-Scores von V ist für eine adäquate Behandlung kein Notarzt erforderlich. 
Ein Patient mit einer isolierten, grob dislozierten, offenen Sprunggelenksfraktur würde dagegen als NACA III eingestuft werden, da eine stationäre Behandlung zwar erforderlich ist, jedoch keine Lebensgefahr besteht. Da unverzüglich eine Reposition unter suffizienter Analgosedierung mit Monitoring der Vitalfunktionen erfolgen muss, ist hier die Notwendigkeit eines Notarzteinsatzes eindeutig gegeben.

Der Zustand des Notfallpatienten kann sich - auch durch das Handeln des Notartzes - sehr dynamisch verändern. Durch eine gute präklinische medizinische Versorgung kann sich der Zustand des Patienten so weit verbessern, dass sich am Ende der Behandlung ein niedrigerer NACA-Score ergibt als zu Behandlungsbeginn. Beispielsweise kann ein Patient mit einem schweren Asthmaanfall oder einer anaphylaktischen Reaktion durch die notärztliche Therapie so weit stabilisiert werden, dass bei Klinikaufnahme keine vitale Gefährdung mehr besteht, während dies vor dem notärztlichen Handeln der Fall war. Aus diesem Grund wird der NACA-Score auf den aktuellsten Einsatzprotokollen sowohl am Anfang als auch am Ende des Einsatzes erhoben und dokumentiert.

Auch wenn die retrospektive Extraktion von NACA-Werten objektiv und ohne jeden Interpretationsspielraum möglich ist, unterliegt die Zuordnung des individuellen NACAWertes der subjektiven Einschätzung des behandelnden Notarztes (Knapp et al. 2008). Sogar vital bedrohliche Krankheitsbilder werden nicht immer mit einem höheren NACA-Punkt eingeordnet.

Aus diesem Grund gab es in Deutschland mehrere Ansätze zur Erstellung eines Scorewertes, der auf objektivierbare Parameter zugreift. So ist der Münchner NACA-Score entstanden, bei dem der klassische NACA-Score mit objektivierbaren Parametern modifiziert wird (Schlechtriemen et al. 2005a). Der Münchner NACA-Score konnte sich jedoch nicht in der Breite durchsetzen, da die klinischen Parameter nicht bei jedem Notfalleinsatz obligat erhoben werden, und ihre nachträgliche Einbeziehung daher sehr aufwendig ist. Dies spiegelt sich auch in der geringen Anzahl an Veröffentlichungen zum Münchner NACA-Score in den letzten Jahrzehnten wider.

Insgesamt lässt der siebenteilige NACA-Score zwar oft, aber keineswegs immer zuverlässige Rückschlüsse darauf zu, ob der Einsatz des Notarztes gerechtfertigt war. Unsere Zahlen demonstrierten eine Korrelation zwischen dem Punktwert des NACA-Scores und dem Anteil medizinisch indizierter Notarzteinsätze, das Problem der Differenzierung und Reproduzierbarkeit bei der Einordnung zwischen NACA II, III und IV besteht jedoch weiterhin (Bernhard et al. 2014). Zudem vermittelt der NACA-Score zwar Informationen über die Schwere des Zustandes des Patienten, aber nicht direkt über das Ausmaß und die Notwendigkeit einer notärztlichen Therapie. Es ist berechtigt anzunehmen, dass schwer verletzte oder kranke Patienten mit höheren NACA-Werten öfter eine intensivere Zuwendung von Personalressourcen benötigen (Schneider et al. 2018), sodass sie von dem Einsatz eines Notarztes profitieren können. Die Arbeitsgruppe von Raatiniemi in Norwegen konnten in einer Analyse von ca. 1500 Fällen aus die Luftrettung einen guten prädiktiven 
Charakter des NACA-Scores hinsichtlich der Mortalität und der Notwendigkeit erweiterter medizinischer Maßnahmen bei der weiteren Behandlung in der Klinik zeigen (Raatiniemi et al. 2013). Diese Ergebnisse bestätigen sich in einer aktuellen Arbeit aus dem Nachbarland Polen (Leszczyński et al. 2019). Leszczyński et al. wiesen bei Patienten mit einem NACAScore IV eine stärkere Assoziation mit vital bedrohlichen Herzrhythmusstörungen während des Transportes ins Krankenhaus nach.

\subsection{Potenzielle Maßnahme zur selektiveren Alarmierung von Notärzten}

Zur Qualitätssicherung im Rettungsdienst wurden bereits 1997 unter anderem Maßnahmen zur korrekten Disposition der entsandten Rettungsmittel durch die Rettungsleitstelle vorgeschlagen (Moecke und Ahnefeld 1997). Die Entwicklung von besseren Selektionskriterien für die Alarmierung eines arztbesetzten Rettungsmittels könnte wegweisend für eine Qualitätssteigerung sein. Zudem könnte eine bessere Ausbildung und mehr praktische Einsatzerfahrung der Disponenten in Rettungsleitstellen zu einem effektiveren Einsatz der vorgehaltenen Rettungsmittel führen (Schlechtriemen et al. 2007).

Vor dem Hintergrund der genannten Überlegungen dieser Diskussion ergeben sich folgenden Ausblicke als potenzielle Maßnahmen zu einer selektiveren Alarmierung von Notärzten:

\subsubsection{Verbesserung der Leistung der Rettungsleitstelle}

\subsubsection{Die Notarzteinsatzkataloge: Überarbeitung und Aktualisierung}

Für das Entsenden eines Notarztes ist der Rettungsleitstellendisponent zuständig. Bei einem eingehenden Notruf wird die Entscheidung über die Alarmierung eines Notarztes anhand von Indikationskatalogen getroffen. Der Notarztindikationskatalog ist eine Handlungsanweisung, seine Anwendung ist im Landesrettungsdienstrecht von einigen Bundesländern verbindlich geregelt (Hennes und Lang 2000). Der Notarztindikationskatalog bietet zum einen Unterstützung für den Disponenten für die Entscheidung welches Rettungsmittel (arztbesetzt vs. nicht arztbesetzt) zu entsenden ist, ermöglicht auch die Standardisierung und gewährt nicht zuletzt dem Leitstellenpersonal die rechtliche Absicherung.

Die großzügige Aufnahme von Leitsymptomen und Notfallsituationen in die Notarztindikationskataloge führt zu einer niederschwelligen Alarmierung des Notarztes, wodurch Notfälle mit einer bestehenden Notarztindikation mit einer hohen Sensitivität erfasst werden (von Kaufmann und Kanz 2012). Dies geht jedoch obligat mit Einbußen in der Spezifität einher, was sich in unseren Ergebnissen widerspiegelte. 
Der Notarztindikationskatalog aus dem Jahr 2001 (BÄK 2001) wurde zwar im Februar 2013 (BÄK 2013) aktualisiert, diese Aktualisierung bestand jedoch nur in der Ergänzung einiger Krankheitsbilder. Besonders erwähnenswert war die Aufnahme des Symptoms "Schmerz" in den Notarztindikationskatalog.

Viele wesentliche Entwicklungen im Rettungsdienst in den letzten Jahren, wie die Einführung des Notfallsanitäters, die bessere Ausstattung der Rettungsmittel und der zunehmende Stellenwert der Telemedizin, fanden in den Einsatzkatalogen des Notarztes jedoch noch keine Berücksichtigung.

In aktuellen Zeiten mehren sich einige Stimmen, wie die Bundesvereinigung der Arbeitsgemeinschaft der Notärzte Deutschlands (BAND), die für die Notwendigkeit einer Reform des Alarmierungssystems für den Notarzt (Reifferscheid 2019) plädieren, damit diese qualifizierte und zunehmend knappe Ressource möglichst effektiv eingesetzt wird.

Eine Überarbeitung des Notarzteinsatzkatalogs mit Berücksichtigung der neuen Entwicklungen in der Notfallmedizin scheint in der naher Zukunft unentbehrlich zu sein (Helm et al. 2017) und soll der erste Schritt zu einer neuen Einsatzstrategie für arztbesetzte Rettungsmittel sein.

Die aktuellen Indikationskataloge sehen die Alarmierung eines Notarztes bei Notfällen wie Hypoglykämie (Rostykus et al. 2016) oder hypertensiver Entgleisung (Wenzel et al. 2014) vor. Beide Situationen sind exemplarische Beispiele für eine Vielzahl von Notfällen, in denen die Patienten mit der Regelkompetenz von Notfallsanitätern ohne Qualitätsverluste behandelt werden können, solange sich der Zustand nicht durch weitere medizinische Erschwernisse kompliziert.

\subsubsection{Die Leitstelle: Optimierung der Abfrage-Algorithmen}

Da die Leitstelle über die Ressource Notarzt disponiert, könnte es sinnvoll sein, eine strukturierte orientierende Notrufabfrage über die Art und Schwere des Notfalls (Lenz et al. 2000) weiterzuentwickeln um die Spezifizität bei der Auswahl der eingesetzten Fahrzeuge zu erhöhen, und insbesondere die notarztbesetzten Rettungsmittel möglichst effektiv einzusetzen (Marung et al. 2013).

Aus kommunikationsbedingten Gründen beim Anrufer und Disponenten (Chien et al. 2019) werden nicht alle Einsätze mit Notarztindikation erkannt, ebenso wie nicht alle Notfälle identifiziert können, bei denen der Einsatz des Notarztes nicht erforderlich ist. Bei den meisten Notfallpatienten fehlen diagnosespezifische Symptome, die am Telefon gut erkannt werden können (Moeck 2013), wie beispielweise bei der Meldung „Verschlechterung Allgemeinzustand“. Ebenfalls nicht vermeidbar sind sich verändernde Zustände zwischen Alarmierung und Eintreffen der Rettungskräfte. Charakteristische Beispiele wären das Sistieren eines Krampfanfalls bzw. die Bewusstlosigkeit bei einem Kollaps. 
Luiz et al. verzeichneten in ihrer aktuellen Untersuchung entgegen der Erwartungen keine Abnahme der Notarztrate trotz Verwendung eines Abfrageprotokolls für eingehende Notrufe (Luiz et al. 2019). In eine ähnliche Richtung argumentiert eine Arbeit von Bohm und Kurland aus dem Jahre 2018, nach deren Ergebnissen für die verschiedene Einsatzprotokolle und Algorithmen wenig wissenschaftliche Evidenz vorhanden ist (Bohm und Kurland 2018).

Die Abfrage der Leitstelle muss daher zwar natürlich gewisse "Kernpunkte" enthalten (wie z. B. Vitalfunktionen und Notfallhergang). Eine individuelle, angepasste Ausgestaltung der Fragen wird dem jeweiligen Notfall jedoch deutlich gerechter als ein starres Schema.

\subsubsection{Arztbesetzung in der Rettungsleitstelle}

Der Arzt ist der höchst qualifizierte Mitarbeiter im Gesundheitssystem, der oft über eine langjährige klinische Erfahrung in verschiedenen Fachrichtungen verfügt. Daher wäre ein möglicher Ansatzpunkt, mit der Anwesenheit eine Arztes in der Leitstelle sowie seiner Einbeziehung in Entscheidungsprozesse möglicherweise zur Steigerung der Qualität und einer besseren Steuerung der Ressourcen beizutragen (Lohs 2016). Dieses Vorgehen wird bereits in Spanien (Miró 2010) und in Portugal (Gomes et al. 2004) praktiziert. In diesen Ländern nimmt ein Arzt mit Erfahrung in der Notfallmedizin in der Leitstelle die Anrufe entgegen, überblickt die Prioritäten der Anrufe, erfasst die Notfalllage, und mittels einer strukturierten, aber angepassten Abfrage entscheidet über die eingesetzten Rettungsmittel und Ressourcen.

Eine arztbesetzte Leitstelle würde zu einer besseren medikolegalen Absicherung der getroffenen Entscheidungen bei der Disposition von Rettungsmitteln sowie zu einer Übernahme von Verantwortung führen (Katzenmeier und Schrag-Slavu 2010). Zudem wäre aus legalen Gründen die Präsenz des Notarztes am Notfallort, zum Beispiel bei Transportverweigerung, nicht immer erforderlich (Fehn 2014).

\subsubsection{Berücksichtigung der aktuellen Entwicklungen im Rettungsdienst: Notfallsanitäter, verbesserte Ausstattung der Rettungsmittel, Telemedizin}

\subsubsection{Konsequente Nutzung des Notfallsanitäters}

Mit der Einführung des Notfallsanitäters wurde nicht nur die Ausbildung einer Gruppe des nicht ärztlichen Personals im Rettungsdienst verbessert, sondern erstmals auch nicht ärztlichem Personal die eigenständige Ausführung von heilkundlichen Maßnahmen, mit einem besonderen Fokus auf die selbständige Durchführung lebensrettender Maßnahmen nach Algorithmen vom sogenannten Pyramidenprozess erlaubt (Lechleuthner und Neupert 2015). Mit der Erweiterung der Kompetenzen des Notfallsanitäters und der Ausarbeitung von standarisierten Vorgehensweisen (engl. SOP - Standard Operating Procedures) werden viele Krankheitsbilder nicht mehr obligat eine Notarztindikation darstellen (DGAI 2016), wie z. B. ein Schlaganfall ohne Bewusstseinsverlust (Soda et al. 2017). 
In diesem Kontext wird bundesweit in zunehmendem Maße die medikamentöse (auch intravenöse) Behandlung akuter Schmerzzustände im Rahmen des Notfallsanitätergesetzes ( $\$ 4$ Abs. 2 Nr. 2c NotSanG), durch Notfallsanitäter ermöglicht (Pfütsch 2019). Infolgedessen bildet die Alarmierung „Schmerz" bei nicht lebensbedrohlichen Zuständen an sich keine Indikationsstellung mehr für den Notarzt, ist aber dennoch Teil der aktuellen Indikationskataloge. Diese Entwicklung wird unter anderem durch eine Untersuchung von Häske gestützt (Häske et al. 2014), nach der eine zufriedenstellende Analgesie unter Anwendung von Algorithmen in nicht lebensbedrohlichen Situationen auch durch nichtärztliches Personal erfolgen kann. Eine weitere Untersuchung von Schempf et al (Schempf et al. 2017) kam zu der Schlussfolgerung, dass bei der Durchführung einer selektiven Analgesie durch Rettungsassistenten und Notärzte ähnliche Sicherheitsgrenzen eingehalten und vergleichbare Behandlungsergebnisse erzielt wurden. Es muss jedoch erwähnt werden, dass sich die genannte Untersuchung auf Analgesie bei isolierten Frakturen der Extremitäten begrenzte, und allgemein schmerzhafte Krankheitsbilder nicht einbezogen wurden. Die fachgerechte Durchführung einer Analgesie durch Rettungs-/ Notfallsanitäter und die daraus resultierende Entlastung der Notärzte kann dazu führen, dass die ohnehin knappe Ressource Notarzt besser für kritische Situationen verfügbar bleibt, die einen Arzt zwingend erfordern. Eine konsequente Nutzung der Regelkompetenz von Notfallsanitätern sollte daher angestrebt werden.

In unklaren Situationen und bei nicht eindeutig gegebener Notarztindikation könnte der mit Notfallsanitätern besetzte RTW primär entsendet werden und sofort mit der Erstversorgung beginnen. Ab diesem Zeitpunkt gäbe es drei mögliche Vorgehensweise: Bei Bedarf könnte der Notarzt umgehend nachgefordert und die Zeit bis zu seinem Eintreffen mit Vorbereitungen für ärztliche Therapiemaßnahmen genutzt werden. Alternativ könnte in bestimmten Situationen die Konsultation eines Telenotarztes erfolgen (Brokmann et al. 2015). Die dritte Option wäre, dass die Notfallsanitäter die erforderlichen medizinischen Maßnahmen im Rahmen ihrer Regelkompetenz selbständig durchführen und den Patienten in die Klinik zur weiteren ärztlichen Versorgung transportieren. Studien weisen auf vergleichbare Behandlungsergebnisse bei ausgewählten Krankheitsbildern hin, wenn nichtärztliches Personal die Patientenversorgung alleine durchführt (Kanz 2018). Dies ist von besonderer Bedeutung bei standarisierten Vorgehensweisen, wie z. B. die Wiederbelebung beim Herzstillstand. Eine große Analyse von Bürger aus dem Deutschen Reanimationsregister unterstützt eindeutig die Aussage, dass kurze Eintreffzeiten und ein schneller Beginn der therapeutischen Maßnahmen den größten Einfluss auf die Behandlungsergebnisse haben (Bürger et al. 2018). Da diese Maßnahmen international durch Algorithmen standarisiert sind und während der Ausbildung intensiv trainiert werden, können sie auch durch nicht ärztliches Personal effektiv durchgeführt werden.

Art und Umfang der medizinischen Maßnahmen (z. B. die Auswahl freigegebener Medikamente), die durch Notfallsanitäter im Rahmen ihrer Regelkompetenz selbständig und ohne Notarzt angewendet werden sollen, werden derzeit lebhaft diskutiert. Die rechtliche 
Lage ist zwar seit 2014 geklärt (Neupert et al. 2014), dennoch unterliegt die praktische Ausgestaltung des Notfallsanitäter-Gesetzes den regionalen ärztlichen Leitern der Rettungsdienste. Somit bestehen zurzeit viele regionale Unterschiede in Hinblick auf die eigenständige Durchführung medizinischer Maßnahmen ohne direkte ärztliche Aufsicht, insbesondere bei der Auswahl freigegebener Medikamente. Vor diesem Hintergrund erscheint es eine interessante Alternative, dass Notfallsanitäter (bzw. Rettungsassistenten) die vorgefundene Lage und den Patientenzustand an einen Telenotarzt zu übermitteln, und dessen Anordnungen im Rahmen der Delegation ausführen, wie in einer Arbeit von Brokmann für den Fall der Analgesie vorgeschlagen (Brokmann et al. 2016).

\subsubsection{Verbesserte Ausstattung der Rettungsmittel}

Ein weiterer Fortschritt ist die überarbeite technische Ausstattung der Rettungswagen. 1999 wurde die ursprüngliche, aus dem Jahr 1967 stammende DIN 75080 für die standarisierte Ausstattung von Rettungsmitteln durch die DIN EN 1789 ersetzt, die eine wesentlich umfangreichere Ausrüstung vorsieht (Holling 2003). Diese Optimierung der Ausrüstung erweitert sowohl die diagnostischen Möglichkeiten als auch die therapeutischen Optionen der präklinischen Patientenversorgung. So ist es zum Beispiel auf jedem Rettungswagen möglich, ein 12-Kanal-Eletrokardiogramm (EKG) durchzuführen (Tanguay et al. 2018), so dass auch Notfallsanitäter einen ST-Hebungs-Myokardinfarkt erkennen können (Carter 2018), wie es im anglo-amerikanischen Rettungssystem der Fall ist. Neue Perspektiven stellen die Entwicklung von einfachen Tests für die schnelle Bestimmung von spezifischen Biomarken durch nicht ärztliches Personal im präklinischen Kontext dar, wie beispielweise die Tests für die Bestimmung der herzspezifischen Troponin-T (Sørensen et al. 2011). Dies könnte dazu führen, dass Notärzte speziell zu akuten Koronarsyndromen gerufen werden und nicht zu anderen unbedenklichen Fällen, wie eine Interkostalneuralgie.

Neben dem Einsatz mechanischer Reanimationshilfen (Treffer et al. 2017) wird die Anwendung diagnostischer Verfahren (engl. Point-of-Care-Diagnostic) wie zum Beispiel die Ultraschalldiagnostik (Heegaard et al. 2010) durch nicht ärztliches Personal (Baron et al. 2019) und die Übertragung der Befunde zur ärztlichen Interpretation diskutiert. Dieses Vorgehen könnte zukünftig neue Möglichkeiten für die Diagnostik und die Behandlung medizinisch relevanter Krankheitsbilder eröffnen, wie z. B. bei freier Flüssigkeit im Abdomen oder bei Patienten mit kardiogenem Schock.

\subsubsection{Die Ausbreitung der Telemedizin}

Die Telemedizin kann die Möglichkeiten der präklinischen Notfallmedizin deutlich erweitern, da der Notarzt seine fachliche Kompetenz einbringen kann, ohne physisch anwesend zu sein. Dieses System ermöglicht diagnostische Maßnahmen, darauf basierende frühzeitige Therapiemaßnahmen und die ärztliche Transportzielauswahl ohne direkte ärztliche Präsenz am Notfallort. Winburn et al. konnten zeigen, dass diverse Notfallsituationen durch den Einsatz eines Telenotarztes sicher abgearbeitet werden konnten 
(Winburn et al. 2018). Anwendungsbereiche mit zunehmender Tragweite für die Telemedizin wäre die präklinische Behandlung von akuten Schmerzen oder Blutdruckentgleisungen, die Einschätzung der Symptome bei apoplektischen Insulten, die Mitbeurteilung von EKGVeränderungen, die Auswahl von Zielkliniken mit geeigneten Fachabteilungen, die ambulante ärztliche Behandlung von Patienten und die Abklärung bei Transportverweigerung (Koncz et al. 2019).

Die Telemedizin ermöglicht neben der Audiokommunikation auch die Übertragung von Echtzeitvitaldaten, von 12-Kanal-EKGs (Rasmussen et al. 2014) sowie Fotos oder Videosequenzen von der Einsatzstelle. Sie könnte daher dazu beitragen, dass arztbesetzte Rettungsmittel seltener primär alarmiert oder vom RTW nachgefordert werden (Brokmann et al. 2017), ohne dass die Qualität der medizinischen Versorgung abnimmt. Darüber hinaus ermöglicht sie die medico-legale Absicherung der Einsatzkräfte (Felzen et al. 2018).

Wie aus der Veröffentlichung von Brokmann et al. hervorgeht (Brokmann et al. 2015), sprechen die Ergebnisse und Erfahrungen der letzten Jahre seit der ersten Implementierung der Telemedizin für eine breitere Anwendung dieser Methode.

Der Telenotarzt kann, ebenso wie ein Arzt auf der Intensivstation, medizinische Maßnahmen an der Einsatzstelle an nichtärztliches Personal delegieren. Mit der weiteren Entwicklung der Technik wird die Telemedizin ihr Spektrum nochmals erweitern (Czaplik et al. 2015), mit der Konsequenz einer besseren Versorgung durch nicht ärztliches Personal unter notärztlicher Fernanleitung (Czaplik und Bergrath 2016).

Selbst wenn die Telemedizin den konventionellen Notarzt nicht ersetzen kann, stellt sie eine hoch effiziente Bereicherung in der notfallmedizinischen Versorgung dar. Pilotstudien aus Aachen illustrieren bereits, dass die Anwendung der Telemedizin eine Abnahme der Einsatzhäufigkeit des konventionellen Notarztes bewirkt (Rossaint et al. 2017). Auf diese Weise ist der Notarzt besser für solche Notfälle verfügbar, bei denen seine Anwesenheit am Patienten und seine manuellen Fertigkeiten gefordert sind, wie z. B. bei Reanimationen oder der Versorgung von Polytrauma-Patienten.

Besonders nennenswert ist die zunehmende Bedeutung des Telenotarztsystems für die präklinische Versorgung im dünn besiedelten ländlichen Raum. Die Arbeit von Süss et al. aus Mecklenburg-Vorpommern belegte einen deutlichen Rückgang der Notarzteinsätze nach Einführung des Telenotarztsystems (Süss et al. 2020).

\subsection{Die unverzichtbare Rolle des Notarztes im Rettungsdienst}

Der Stellenwert des Notarztes im deutschen Rettungssystem ist durch wissenschaftliche Evidenz belegt. Fischer et al. verglichen in einer Studie vier Rettungsdienstsysteme: Das deutsche und spanische (arztbasiert) auf der einen Seite, sowie das englische und amerikanische (mit ausschließlich nichtärztlichem Personal, engl. Paramedics) auf der anderen Seite. Bei Herz-Kreislaufstillständen sowie bei Krankheitszuständen wie Dyspnoe oder 
Brustschmerzen waren die Behandlungsergebnisse der ärztlich besetzten Rettungsdienste den nicht ärztlichen Systemen überlegen (Fischer et al. 2011).

$\mathrm{Zu}$ ähnlichen Ergebnissen kamen Böttinger et al. in einer systematischen Übersichtsarbeit (engl. systematic review): Wurden bei präklinischen Herz-Kreislaufstillständen die Reanimationsmaßnahmen durch einen Notarzt geleitet, waren die Behandlungsergebnisse bezogen auf das Wiedereinsetzen eines Spontankreislaufes (engl. ROSC) und der Überlebenswahscheinlichkeit nach 30 Tagen im Vergleich zu nicht ärztlich geleiteten Reanimationen besser (Böttiger et al. 2016).

Die Bedeutung des Notarztes bei der präklinischen Notfallversorgung wird außerdem durch eine aktuelle Veröffentlichung von Knapp et al. unterstrichen. In dieser Übersichtsarbeit konnten eindeutig positive Effekte des Notarztes auf die Behandlungsergebnisse und das Überleben nach schwerem Trauma gezeigt werden, insbesondere wenn der Einsatz mittels Luftrettung durchgeführt wurde (Knapp et al. 2019).

Des Weiteren konnten Chen et al. zeigen, dass Überleben von Trauma-Patienten mit abnormaler Herzfrequenz, einem Glasgow-Coma-Score $\leq 8$ oder einem Hämato/Pneumothorax signifikant besser war, wenn die Patienten von einem Notarzt versorgt und mit dem RTH in ein geeignetes Krankenhaus transportiert wurden, und zwar auch in solchen Fällen, in denen die Versorgung ohne Notarzt und der bodengebundene Transport in das nächstliegende Krankenhaus weniger Zeit in Anspruch genommen hätte. Diese Ergebnisse zeigten, dass die Expertise eines Notarztes für ein erweitertes Atemwegsmanagement und die Versorgung von Thoraxtraumata eine wichtigere Rolle spielt als der schnellstmögliche Transport in das nächste erreichbare Krankenhaus (Chen et al. 2018). Die Überlegenheit einer präklinischen ärztlichen Behandlung ist noch ausgeprägter, wenn Anästhesisten als Notärzte eingesetzt werden, da diese Berufsgruppe durch ihre tägliche Arbeit über ein hohes $\mathrm{Maß}$ an Routine bei der Durchführung lebenserhaltender Maßnahmen verfügt (Mikkelsen et al. 2015).

Im Zusammenhang mit Möglichkeiten zur Kosteneinsparung im Gesundheitswesen stellt sich dagegen die Frage, ob der Rettungsdienst in Zukunft ohne Notärzte funktionieren könnte (Sefrin und Kuhnigk 2006). Seit einigen Jahren werden Untersuchungen durchgeführt, um zu zeigen wie sicher die Anwendung von verschiedenen Medikamenten durch nichtärztliches Personal sein kann (Luiz et al. 2015).

In diesem Zusammenhang muss jedoch erwähnt werden, dass selbst in europäischen Ländern mit Rettungssystemen, die traditionell auf gut ausgebildeten Rettungs- bzw. Notfallsanitätern basieren, im letzten Dezennium der Bedarf an einer kompetenten präklinischen notärztlichen Versorgung erkannt wurde (Gretenkort et al. 2017).

Das Rettungssystem von Dänemark basiert auf nicht ärztlichem Personal, an manchen Standorten steht jedoch ein Notarzt zur Unterstützung zu Verfügung. Hamilton et al. konnten in einer Studie belegen, dass die Ergebnisse der prähospitalen 
Reanimationsmaßnahmen und die Überlebenswahrscheinlichkeit nach 30 Tagen bei notärztlich geleiteten Reanimationen besser sind als in Fällen, in denen die Reanimationsmaßnahmen ausschließlich durch nicht ärztliches Personal erfolgten (Hamilton et al. 2016).

Sogar in Großbritannien, der "Wiege" der anglo-amerikanischen Paramedics (engl. medizinisch-technisch-Assistent), konnten in einer klinischen Studie mehrfache Vorteile belegt werden, wenn Notfallmediziner bereits in die präklinischen Versorgung von Traumapatienten involviert wurden (Roberts et al. 2009). In den Niederlanden und Norwegen (Lossius et al. 2002), jedoch auch in außereuropäischen Ländern wie Japan (Igarashi et al. 2018), wurden infolge der wissenschaftlichen Erkenntnisse die nicht ärztlich basierten Rettungsdienstsysteme mit Notärzten ergänzt, die nicht nur auf Rettungshubschraubern, sondern auch auf bodengebundenen Fahrzeugen eingesetzt werden.

Obwohl sich die Ausbildung des nicht ärztlichen Personals in der Vergangenheit sowohl qualitativ als auch quantitativ stetig verbessert hat, ist nicht ärztliches Personal bei der Behandlung von Notfallpatienten stets an Algorithmen gebunden (Jansen et al. 2018). Sowohl die Patienten als auch die Situationen, mit denen das Personal im Rettungsdienst konfrontiert wird, sind in einer Vielzahl der Fälle komplex und lassen sich durch "Standards" nur unzureichend abbilden. Zu erwähnen wären z. B. die Extreme des Lebensalters, d. h. Kinder (Tiesmeier et al. 2016) und Senioren (Duong et al. 2017): Diese können eine sehr variable Sensibilität auf Schmerzmittel zeigen, weshalb für einen sicheren Umgang mit starken analgetischen Substanzen eine erhebliche Expertise erforderlich ist, um diese im Rahmen einer komplexen präklinischen Behandlung zum Vorteil des Patienten einzusetzen (Luger und Luger 2017). Eine andere häufige Klientel im Rettungsdienst sind Patienten mit Alkohol-, Medikamenten- und Substanzabusus (Eyer und Zilker 2012 und Kreimeier und Arntz 2012), bei denen es ebenfalls zu unvorhersehbaren, gravierenden Interaktionen mit Notfallmedikamenten kommen kann (Beerhorst et al. 2012). Qualifizierte Notärzte können auch in solchen Situationen Diagnosen stellen, Entscheidungen treffen und individuelle Behandlungen durchführen, die aufgrund ihrer Komplexität mit starren Algorithmen nur sehr unzureichend abgedeckt wären.

Zusammenfassend belegt eine Vielzahl wissenschaftlicher Arbeiten, dass in bestimmten Situationen der präklinische Einsatz eines versierten Notfallmediziners zu einem messbaren Überlebensvorteil führen kann. Der Notarzt sollte daher obligat bei der Therapie von instabilen Patienten, zur Analgosedierung mit Gefährdung des Atemweges, für eine invasive und auch eine nicht invasive Beatmung sowie in komplizierten Fällen alarmiert werden (Luiz et al. 2016), ebenso bei ST-Hebungsinfarkten und zur Behandlung der akuten und schweren Atemnot, um einige zu nennen.

Wie Bollinger et al. 2020 feststellten, besteht noch für viele invasive Maßnahmen bei nichtärztlichem Personal ein gravierender Ausbildungsbedarf (Bollinger et al. 2020). Da 
manuelle Tätigkeiten (insbesondere unter erschwerten äußern Umständen) jedoch nur dann sicher beherrscht werden, wenn sie regelmäßig, intensiv und gründlich trainiert werden, dürften Notfallsanitäter nur sehr selten oder niemals über eine ausreichende praktische Routine verfügen, um Verfahren wie Thoraxdrainagen oder die Intubation von Kindern sicher durchführen zu können.

Die Notkompetenzen sollen weiterhin als notfallmäßige Maßnahmen bis zum Eintreffen des Notarztes bestehen bleiben. Jedoch ist eine ärztliche Unterstützung bei komplexen bzw. lebensbedrohlichen Situationen unverzichtbar und soll weiterhin jederzeit verfügbar sein. Eine Nachforderung des Notarztes ist nach unseren Ergebnissen sinnvoll und sollte beibehalten werden.

Eine kontinuierliche Weiterbildung (Reifferscheid et al. 2016) für den Notarzt ist essentiell, um die notärztlichen Fähigkeiten auf einem konstant hohen Niveau zu halten. Heutzutage ist die Simulation ein wesentliches Element dieser notärztlichen Weiterbildung (Gal et al. 2016).

Neue technische Entwicklungen in der Notfallmedizin wie z. B. die Telemedizin könnten dazu beitragen, dass ein deutlich größerer Anteil der Patienten auch ohne einen anwesenden Notarzt versorgt wird und die Einsatzhäufigkeit von Notärzten dadurch abnimmt. Hierdurch könnte der Horizont möglicher Einsatzindikationen für den Notarzt erweitert werden, denkbar wäre z. B. die Anwendung von extrakorporalen kreislaufunterstützenden Verfahren in der prähospitalen Phase, wie die Anlage ECLS- (engl. Extracorporeal Life Support) oder ECMO-Systemen (engl. Extracorporeal Membrane Oxygenation) (Lamhaut et al. 2017).

\subsection{Limitationen dieser Studie.}

Die wesentlichste Limitation der Untersuchung besteht in der geringen Spezifität, die unser Schema zur Beurteilung der Notarztindikation erzielt. Es wurde zwar erhoben ob eine ärztliche Maßnahme (i. d. R. eine Medikamentengabe) erfolgte. Um den Einfluss subjektiver Interpretationsmöglichkeiten zu minimieren wurde jedoch nicht differenziert, ob diese Maßnahmen zeitkritisch waren oder ggf. auch erst im Krankenhaus hätten eingeleitet werden können. Beispielsweise kann der Notarzt zu einem jungen Patienten mit einem Infekt aber ohne vitale Bedrohung entsandt werden und eine antiphlogistische Medikation verabreichen. $\mathrm{Da}$ in unserer Untersuchung alle Medikamente gleich bewertet wurden, würde dieser Einsatz nach unseren Kriterien als medizinisch indiziert eingestuft. Retrospektiv betrachtet scheint es jedoch fragwürdig, ob der Einsatz des Notarztes tatsächlich zwingend notwendig war; die Situation in diesem Beispiel würde als NACA Stufe I oder II (geringgradige Störung) eingeordnet werden. Im Falle einer alleinigen Alarmierung eines RTW hätte die Besatzung den Patienten mit hoher Wahrscheinlichkeit ohne Notarzt sachgerecht versorgt, alternativ hätte z. B. auf den kassenärztlichen Bereitschaftsdienst zurückgegriffen werden können. Der Anteil an Patienten, die auch ohne einen Notarzt qualifiziert hätten versorgt werden können, 
dürfte daher wesentlich höher liegen als die 29\% der Fälle, in denen die anwesenden Notärzte keine originär ärztlichen Maßnahmen durchgeführt haben.

Eine weitere Limitation der Untersuchung besteht darin, dass das zweite Kriterium zur Beurteilung der Notarzt-Indikation, nämlich die Bewertung der medizinischen Notwendigkeit einer ärztlichen Transportbegleitung, von der subjektiven Einschätzung des behandelnden Notarztes abhing. Wie unsere Ergebnisse zeigten, war diese Einschätzung auch von dessen Erfahrungshorizont abhängig.

Drittens ist einschränkend für die allgemeine Gültigkeit dieser Untersuchung zu erwähnen, dass Notärzte in Deutschland keiner einheitlichen Fachrichtung angehören (Reifferscheid und Harding 2017). Die arztbesetzen Rettungsmittel in Göttingen werden jedoch ausschließlich mit Ärzten der Klinik für Anästhesiologie besetzt, wodurch die Repräsentativität dieser Untersuchung möglicherweise eingeschränkt wird. Eine Publikation von Sollid und Rehn unterstreicht die Tatsache, dass Anästhesisten aufgrund ihres innerklinischen Aufgabenspektrums und den daraus resultierenden Fertigkeiten am besten für die präklinische Versorgung von Notfallpatienten qualifiziert sind (Sollid und Rehn 2017). Einen besonderen Stellenwert hat dabei die präklinische Sicherung des Atemweges, die zu den Kernkompetenzen von Anästhesisten zählt (Rognås et al. 2013). Eine Arbeit von Schaller et al. konnte darüber hinaus signifikante Unterschiede zwischen Anästhesisten und Chirurgen bei der präklinischen analgetischen Behandlung belegen (Schaller et al. 2019). Für zukünftige Studien hinsichtlich unserer Fragestellungen wäre daher auch eine differenzierte Betrachtung von Notärzten aus verschiedenen Fachrichtungen interessant.

Zudem muss bei der detaillierteren Betrachtung der Ergebnisse spezieller Einsatzmeldungen, wie insbesondere bei psychiatrischen Notfällen, die anteilig geringere Fallzahl berücksichtigt werden.

\subsection{Schlussfolgerungen und Ausblicke für die Zukunft}

Die Unterscheidung zwischen Notfällen, die einen Notarzt zwingend erfordern und Patienten, die ggf. auch von nichtärztlichem Personal effektiv und sicher behandelt werden können, ist von essentieller Bedeutung. In dieser Arbeit wurde nachgewiesen, dass in fast einem Drittel der Einsätze der Notarzt keine explizit ärztliche Maßnahme durchführte. Das hierauf basierende Bewertungssystem, mit dem Notarzteinsätze in dieser Studie als indiziert oder nicht indiziert eingestuft wurden, ist sehr sensitiv, aber wenig spezifisch. Es kann daher angenommen werden, dass eine adäquate Patientenversorgung in einem relevanten Teil derjenigen Fälle, in denen der Notarzteinsatz als indiziert gewertet wurde, auch durch Notfallsanitäter ohne Notarzt möglich gewesen wäre.

Mögliche Alternativen für den Einsatz des Notarztes bestehen in der Telemetrie (d. h. Übertragung von technischen Befunden wie z. B. EKG), der Einbeziehung eines TeleNotarztes sowie im Ausbau der Regelkompetenzen von Notfallsanitätern, diese Ansätze 
weisen jedoch noch weiteren Forschungsbedarf auf. Im deutschen Rettungssystem wird der Notarzt auch weiterhin eine unverzichtbare Rolle spielen (Gretenkort et al. 2019), die sich in der Zukunft jedoch parallel zu den technischen, medizinischen und strukturellen Entwicklungen weiter wandeln wird (Gries et al. 2017). Vor dem Hintergrund potenzieller Nachteile einer liberalen Alarmierung sowie der zunehmend knappen Ressource "Notarzt", sollten fokussierter Einsatzstrategien für den Notarzt entwickelt werden, um eine stabile Versorgungsqualität im Rettungsdienst zu gewährleisten.

Bislang existieren nur wenige Studien im deutschsprachigen Raum, die die Indikation von Notarzteinsätzen thematisieren. Da randomisierte, kontrollierte Studien zu diesem Thema aus ethischen Gründen nicht vertretbar sind, sollten weitere prospektiven Untersuchungen über das Leistungsspektrum von Notärzten erfolgen. Eine optimale Zusammenstellung des Rettungsteams hinsichtlich der medizinischen Besetzung sowie Kommunikation unter den Teammitgliedern und die Delegation von Aufgaben wären Felder zukünftiger Untersuchungen.

Die Position des Notfallmediziners vor dem Hintergrund konstanter Fortschritte im Rettungsdienst wird voraussichtlich Thema der weiterführenden Forschung in der Zukunft sein. 


\section{$5 \quad$ Zusammenfassung}

Das Rettungsdienstsystem in Göttingen (und in ganz Deutschland) ist dual gestaltet: Fahrzeuge ohne Notarzt (Rettungswagen - RTW) und ärztlich besetzte Rettungsmittel (Notarzteinsatzfahrzeug - NEF oder Rettungshubschrauber - RTH) werden parallel durch die Leitstelle alarmiert und treffen sich beim Rendezvous-System am Einsatzort. Wird der RTW alleine zum Einsatz entsendet, kann - falls notwendig - der Notarzt nachgefordert werden. Im Rahmen der Notkompetenz können lebensrettende medizinische Maßnahmen auch von nicht ärztlichem Personal eigenständig durchgeführt werden, wenn das Eintreffen des Notarztes nicht abgewartet werden kann.

Die Häufigkeit von Notarzteinsätzen hat in den letzten Jahrzehnten in erheblichem Umfang zugenommen. Die Entscheidung durch die Rettungsleitstelle über den Einsatz eines Notarztes wird anhand von Indikationskatalogen mit Einsatzstichwörtern getroffen. Diese Strategie wurde, trotz Hinweisen auf potenzielle Nachteile, bislang kaum ernsthaft in Frage gestellt. Es wurde daher eine prospektive Analyse durchgeführt, in der Notarzteinsätze in medizinisch indiziert und nicht medizinisch indiziert unterteilt wurden. Einteilungskriterium war die Durchführung einer originär ärztlichen Maßnahme, d. h. entweder eine notärztliche Transportbegleitung aus medizinischen Gründen oder die Gabe von Medikamenten.

In dieser Arbeit wurde nachgewiesen, dass in fast einem Drittel der Einsätze der Notarzt keine der genannten Maßnahmen durchführte. Die bisherige Bewertung für die Indikation eines Notarzteinsatzes mittels des NACA-Scores ist zwar orientierend, die in dieser Studie an häufigsten vorkommende Punktzahl NACA III ist aber für diesen Zweck nicht aussagekräftig. Eine weitere Betrachtung der Ergebnisse dieser Untersuchung veranschaulicht die Notwendigkeit der Überarbeitung der Alarmierungsstrategie für arztbesetzte Rettungsmittel in den Leitstellen, um einen selektiveren Einsatz von Notärzten zu erzielen. Ein möglicher Ansatz wäre die Überarbeitung der Notarzteinsatzkataloge, die durch neue Entwicklungen in der Notfallmedizin wie z. B. Telemedizin und die Regelkompetenzen von Notfallsanitätern möglich wäre. Die Versorgung von komplex traumatisierten Patienten und Kindernotfälle sollten nach wie vor obligate Einsatzindikationen für den Notarzt bleiben. Für die Mehrheit der nicht traumatologischen Notfälle könnten jedoch die Einsatzkriterien überdacht werden. Besonders exemplarisch sind die psychiatrischen Notfälle. Obwohl bei der Auswahl arztbesetzter Rettungsmittel (NEF oder RTH) einsatzstrategische Gründe die Hauptrolle spielen, zeigen unsere Ergebnisse, dass der Rettungshubschrauber selektiver eingesetzt wird. Durch ihre Regelkompetenz könnten Notfallsanitäter in vielen akuten Situationen auch ohne Notarzt selbständig handeln, und somit eine Filterfunktion für eine gezielte Nachalarmierung des Notarztes übernehmen, wie unsere Daten zeigen.

Für komplexe Notfallsituationen ist belegt, dass der Einsatz des Notarztes zu besseren Überlebensraten und weniger ausgeprägten Folgeerscheinungen nach einem Notfallereignis führt. In der präklinischen Versorgung von Notfallpatienten wird der Notarzt als 
höchstqualifizierteste Instanz zwar auch in Zukunft unverzichtbar bleiben, seine Rolle im Rettungsdienst wird sich aber vor dem Hintergrund aktueller Entwicklungen in der Notfallmedizin wandeln. 


\section{$6 \quad$ Anhang}

\subsection{Fragebogen dieser Untersuchung für den Notarzt}

\begin{tabular}{|c|c|c|c|c|c|c|c|c|}
\hline Datum & Einsatz-Nr. & $\begin{array}{l}\text { Einsatzstichwort } \\
\text { (It. Meldung) }\end{array}$ & 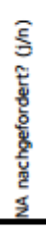 & Zielklinik & 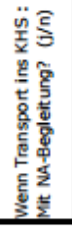 & 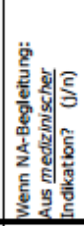 & 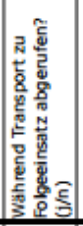 & $\begin{array}{l}\text { Rettungsmittel } \\
\text { (NEF/RTH, Rufname) }\end{array}$ \\
\hline & & & & & & & & \\
\hline & & & & & & & & \\
\hline & & & & & & & & \\
\hline & & & & & & & & \\
\hline & & & & & & & & \\
\hline & & & & & & & & \\
\hline & & & & & & & & \\
\hline & & & & & & & & \\
\hline & & & & & & & & \\
\hline & & & & & & & & \\
\hline
\end{tabular}

Bei Rückfragen: Mauricio Arnoldt (919-4158) oder Daniel Heise (919-4069) 


\subsection{Notarztprotokoll DIN für Göttingen}

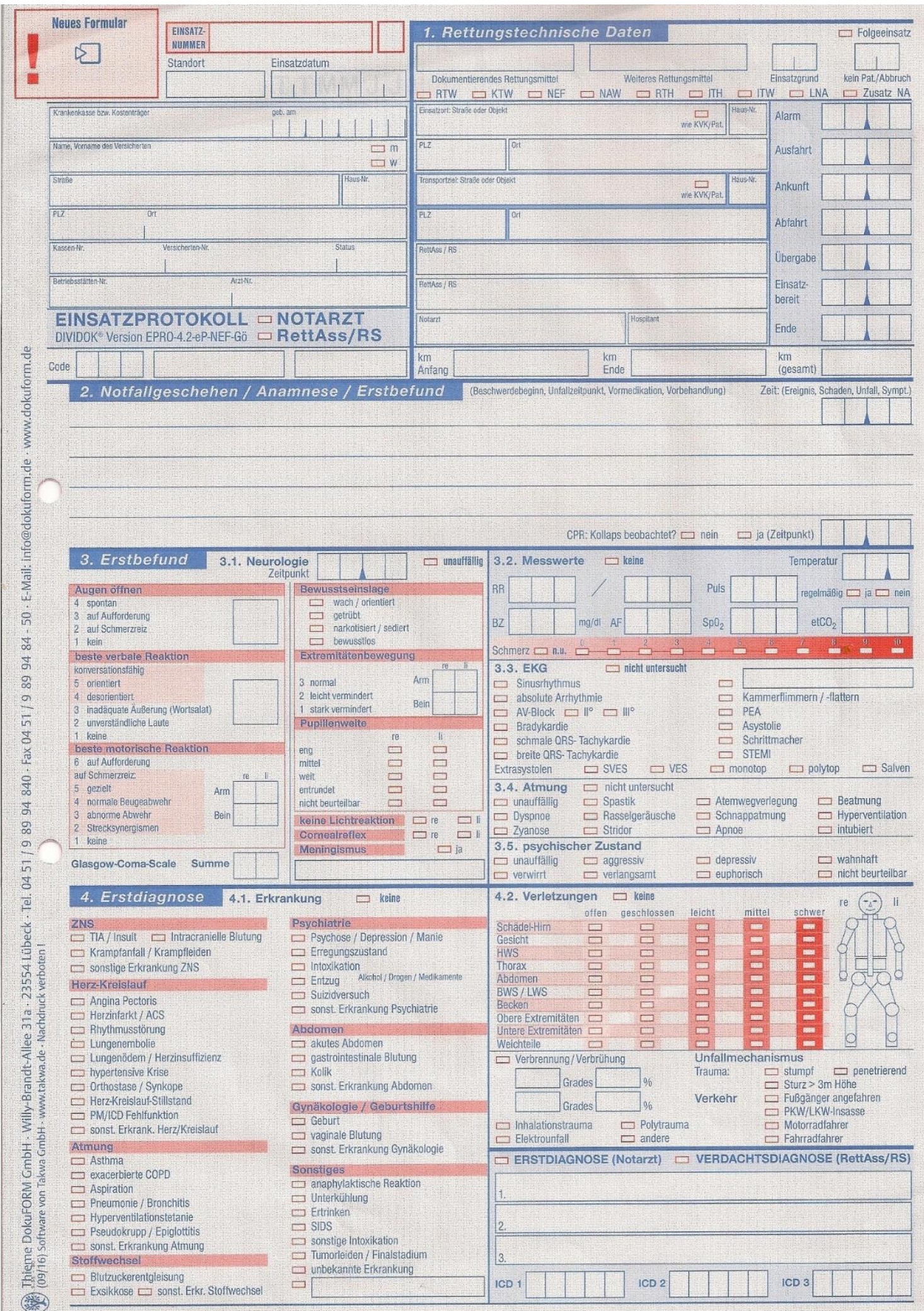




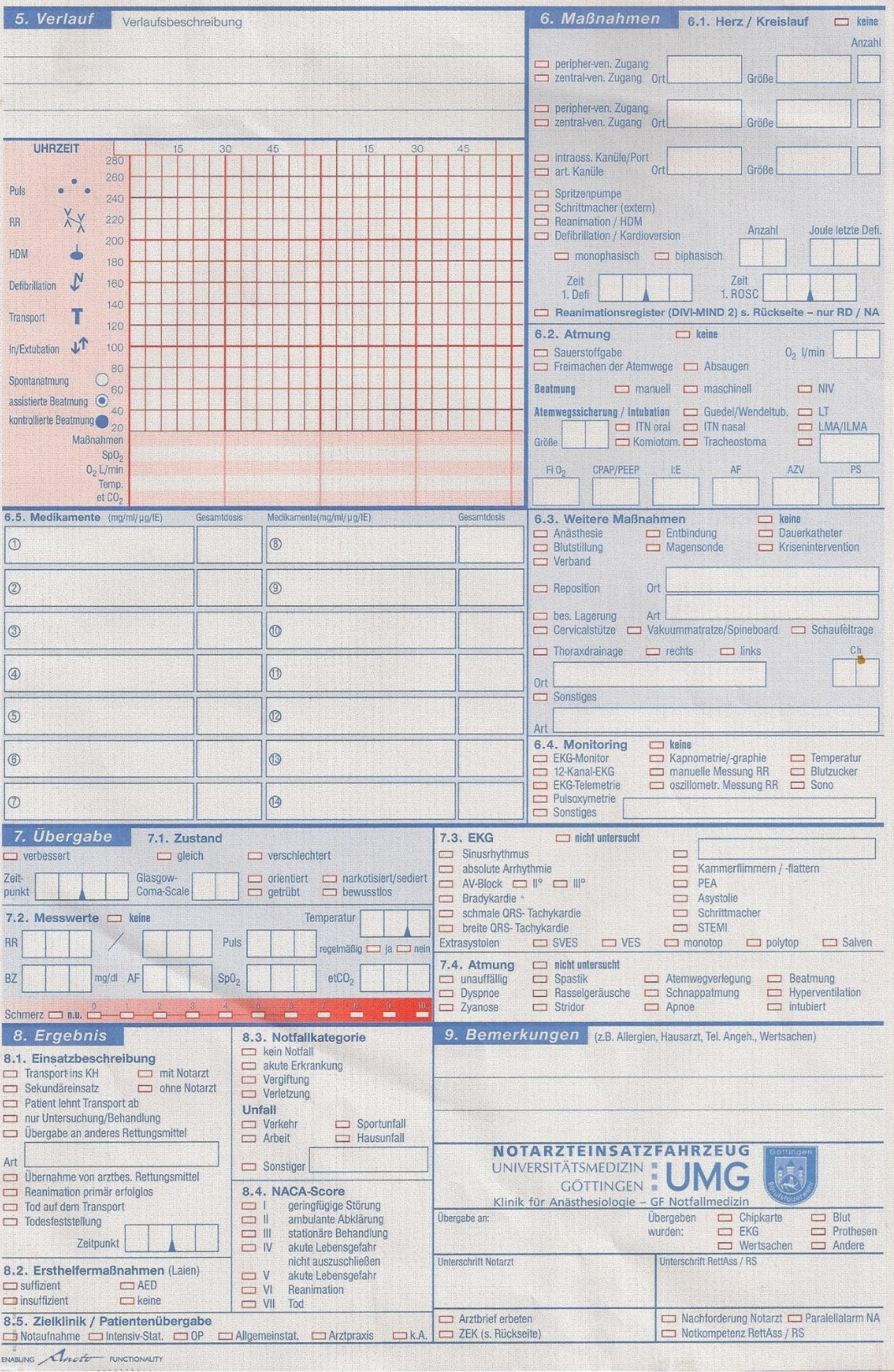




\section{$7 \quad$ Literaturverzeichnis}

Ahnefeld FW (2003): Notfallmedizin gestern. Anaesthesiol Intensivmed Notfallmed Schmerzther $\underline{38}, 277-281$

Ahnefeld FW, Altemeyer KH, Dick WF, Dirks B, Lackner CK, Stratmann D (2003): Die personelle Situation im Rettungsdienst. Notf Rett Med $\underline{6}, 526-532$

Alessandrini H, Oberladstätter D, Trimmel H, Jahn B, Baubin M (2012): NACA-Scoringsystem. Notf Rett Med 15, 42-50

Al-Shaqsi S (2010): Models of international emergency medical service (EMS) Systems. Oman Med $\mathrm{J} 2 \underline{25}, 320-323$

Andruszkow H, Frink M, Zeckey C, Krettek C, Hildebrand F, Mommsen P (2012): Merits and capabilities of helicopter emergency medical service (HEMS) in traumatized patients. Technol Health Care 20, 435-444

Andruszkow H, Lefering R, Frink M, Mommsen P, Zeckey C, Rahe K, Krettek C, Hildebrand F (2013): Survival benefit of helicopter emergency medical services compared to ground emergency medical services in traumatized patients. Crit Care 17, R124

Andruszkow H, Schweigkofler U, Lefering R, Frey M, Horst K, Pfeifer R., Beckers SK, Pape HC, Hildebrand F (2016): Impact of helicopter emergency medical service in traumatized patients: which patient benefits most? PloS ONE 11 , e0146897

Armbruster W, Kubulus D, Schlechtriemen T, Adler J, Höhn M, Schmidt D, Duchêne S, Steiner P, Volk T, Wrobel M (2014): Verbesserung der Notarztausbildung durch Simulatortraining. Anaesthesist 63, 691-696

Aruga Y (2013): Emergency medical services for the elderly: present fact and future challenge. Nippon Rinsho 71, 964-968

Aschenbrenner U, Neppl S, Ahollinger F, Schweigkofler U, Weigt JO, Frank M, Zimmermann M, Braun J (2015): Einsatz der Luftrettung in der Nacht. Unfallchirurg 118, 549-563

Bader K, Bernhard M, Gries A, Kaul M, Schröder R, Ramshorn-Zimmer A (2018): Entwicklung bodengebundener Notarzteinsätze im Stadtgebiet Leipzig von 2003 bis 2013. Anaesthesist 67, $177-187$

Baron A, Beirne G, Wald A (2019): Paramedic point of care ultrasound at Australian mass gatherings. Australas J Ultrasound Med 22, 56-60

Bartels U (2001): Kindernotfälle. Notarzt 17, 31-36

Bayer J, Pache G, Hammer TO, Zwingmann J, Südkamp NP, Strohm PC (2013): German trauma centers: level-dependent differences in polytrauma care regarding resources and diagnostic concepts. Chirurg 84, 316-321

BÄK (2001): Indikationskatalog für den Notarzteinsatz: Unter Bezug auf den Patientenzustand. Dtsch Arztebl 98, A3467

BÄK (2013): Indikationskatalog für den Notarzteinsatz: Handreichung für Telefondisponenten in Notdienstzentralen und Rettungsleitstellen. Dtsch Arztebl 110, A521 
Bedarfsplan Rettungsdienst Stadt Göttingen (2015).

https://ratsinfo.goettingen.de/bi/vo020.asp?VOLFDNR=12323, abgerufen am 21.01.2020

Beerhorst KS, Kardels B, Beine KH (2012): Medical Clearance bei psychiatrischen Symptomen. Notf Rett Med 15, 338-341

Behrendt H, Schmiedel R, Auerbach K (2009): Überblick über die Leistungen des Rettungsdienstes in der Bundesrepublik Deutschland im Zeitraum 2004/05. Notf Rett Med 12, 383-388

Bernhard M, Hilger T, Sikinger M, Hainer C, Haag S, Streitberger K, Martin E, Gries A (2006): Patientenspektrum im Notarztdienst: Was hat sich in den letzten 20 Jahren geändert? Anaesthesist 55, 1157-1165

Bernhard M, Trautwein S, Stepan R, Zahn P, Greim CA, Gries A (2014): Notärztliche Einschätzung der klinischen Weiterversorgung von Notfallpatienten. Anaesthesist $\underline{63}, 394$ 400

Bohm K, Kurland L (2018): The accuracy of medical dispatch - a systematic review. Scand J Trauma Resusc Emerg Med 26, 94

Bollinger M, Langner M, Wellershaus D, Kaisers W, Russo SG (2020): Durchführung invasiver ärztlicher Maßnahmen durch Notfallsanitäter. Notf Rett Med 23, 23-36

Böttiger BW, Bernhard M, Knapp J, Nagele P (2016): Influence of EMS-physician presence on survival after out-of-hospital cardiopulmonary resuscitation: systematic review and metaanalysis. Crit Care $\underline{20}, 4$

Brokmann JC, Dönitz S, Bischoni R, Gliwitzky B, Flake F (2013): Notfallsanitätergesetz. Notf Rett Med 16, 604-610

Brokmann JC, Rossaint R, Bergrath S, Valentin B, Beckers SK, Hirsch F, Jeschke S, Czaplik M (2015): Potenzial und Wirksamkeit eines telemedizinischen Rettungsassistenzsystems. Anaesthesist 64, 438-445

Brokmann JC, Rossaint R, Hirsch F, Beckers SK, Czaplik M, Chowanetz M, Tamm M, Bergrath S (2016): Analgesia by telemedically supported paramedics compared with physicianadministered analgesia: A prospective, interventional, multicentre trial. Eur J Pain 20, 1176-1184

Brokmann JC, Felzen M, Beckers SK, Czaplik M, Hirsch F, Bergrath S, Rossaint R (2017): Telemedizin: Potenziale in der Notfallmedizin. Anästhesiol Intensivmed Notfallmed Schmerzther $\underline{52}, 107-117$

Brown JB, Leeper CM, Sperry JL, Peitzman AB, Billiar TR, Gaines BA, Gestring ML (2016): Helicopters and injured kids: Improved survival with scene air medical transport in the pediatric trauma population. J Trauma Acute Care Surg $\underline{80}$, 702-710

Brown J, Sajankila N, Claridge JA (2017): Prehospital assessment of trauma. Surg Clin North Am 97, 961-983

Buis ML, Maissan IM, Hoeks SE, Klimek M, Stolker RJ (2016): Defining the learning curve for endotracheal intubation using direct laryngoscopy: A systematic review. Resuscitation $\underline{99}$, 63-71 
Bürger A, Wnent J, Bohn A, Jantzen T, Brenner S, Lefering R, Seewald S, Gräsner J-T, Fischer M (2018): The effect of ambulance response time on survival following out-of-hospital cardiac arrest. Dtsch Arztebl Int 115, 541-548

Burgkhardt M, Burgkhardt A (2007): Vom dringlichen ärztlichen Hausbesuch zum Notarztdienst Eine historische Betrachtung. Notfall \& Hausarztmedizin $\underline{33}$, 532-535

Buschmann C, Poloczek S, Giesecke MT, Kleber C (2013): Vermeidbare Todesfälle nach Trauma. Notarzt $\underline{29}, 91-98$

Carron P-N, Dami F, Yersin B, Toppet V, Burnand B, Pittet V (2015): Increasing prehospital emergency medical service interventions for nursing home residents. Swiss Med Wkly $\underline{145}$, w14126

Carter AJE (2018): ST-segment elevation myocardial infarction (STEMI) bypass: The importance of paramedics in an integrated STEMI system of care. CJEM 20, 813-815

Chen J, Bellomo R, Flabouris A, Hillman K, Finfer S, MERIT Study Investigators for the Simpson Centre, ANZICS Clinical Trials Group (2009): The relationship between early emergency team calls and serious adverse events. Crit Care Med $\underline{37}, 148-153$

Chen X, Gestring ML, Rosengart MR, Billiar TR, Peitzman AB, Sperry JL, Brown JB (2018): Speed is not everything: Identifying patients who may benefit from helicopter transport despite faster ground transport. J Trauma Acute Care Surg $\underline{84}$, 549-557

Chien CY, Chien WC, Tsai LH, Tsai SL, Chen CB, Seak CJ, Chou YS, Ma M, Weng YM, Ng CJ, et al. (2019): Impact of the caller's emotional state and cooperation on out-of-hospital cardiac arrest recognition and dispatcher-assisted cardiopulmonary resuscitation. Emerg Med J $\underline{36}$, 595-600

Churpek MM, Edelson DP, Lee JY, Carey K, Snyder A, et al. (2017): Association between survival and time of day for rapid response team calls in a national registry. Crit Care Med $\underline{45}$, $1677-1682$

Corfield AR, Silcock D, Clerihew L, Kelly P, Stewart E, Staines H, Rooney KD (2018): Paediatric early warning scores are predictors of adverse outcome in the pre-hospital setting: A national cohort study. Resuscitation $\underline{133}$, 153-159

Czaplik M, Bergrath S: Telemedizin in der Notfallmedizin. In: Fischer F, Krämer A (Hrsg.): eHealth in Deutschland: Anforderungen und Potenziale innovativer Versorgungsstrukturen. Springer, Berlin, Heidelberg 2016, 319-333

Czaplik M, Brokmann J, Hochhausen N, Beckers SK, Rossaint R (2015): Heutige Möglichkeiten der Telemedizin in der Anästhesiologie. Anaesthesist 64, 183-189

Desmettre T, Yeguiayan JM, Coadou H, Jacquot C, Raux M, Vivien B, Martin C, Bonithon-Kopp C, Freysz M, the French Intensive Care Recorded in Severe Trauma (2012): Impact of emergency medical helicopter transport directly to a university hospital trauma center on mortality of severe blunt trauma patients until discharge. Critical Care 16, R170

DGAI (2016): Telemedizin in der prähospitalen Notfallmedizin: Strukturempfehlung der DGAI. Anästh Intensivmed $\underline{57}, 160-166$

Dick WF (2006): Martin Kirschner: 1879-1942-A surgeon in prehospital care. Resuscitation $\underline{68}$, 319-321

Dick WF (2002): Friedrich Wilhelm Ahnefeld. Resuscitation $\underline{53}$, 247-249 
Dick WF (2003): Anglo-American vs. Franco-German emergency medical services system. Prehosp Disaster Med 18, 29-37

Doggen CJM, Zwerink M, Droste HM, Brouwers PJAM, van Houwelingen GK, van Eenennaam FL, Egberink RE (2016): Prehospital paths and hospital arrival time of patients with acute coronary syndrome or stroke, a prospective observational study. BMC Emerg Med 16, 3

Duong HV, Herrera LN, Moore JX, Donnelly J, Jacobson KE, Carlson JN, Mann NC, Wang HE (2018). National Characteristics of Emergency Medical Services Responses for Older Adults in the United States. Prehosp Emerg Care 22, 7-14

Eastwood K, Morgans A, Stoelwinder J, Smith K (2018): Patient and case characteristics associated with 'no paramedic treatment' for low-acuity cases referred for emergency ambulance dispatch following a secondary telephone triage: a retrospective cohort study. Scand J Trauma Resusc Emerg Med 26, 8

Ebmeyer U, Röse W (2013): Development of the physician based prehospital emergency medicine system in the former GDR. Anasthesiol Intensivmed Notfallmed Schmerzther 48, 730-733

Eich C, Roessler M, Timmermann A, Heuer JF, Gentkow U, Albrecht B, Russo SG (2009): Präklinische Kindernotfälle. Anaesthesist $\underline{58}$, 876-883

Engel P, Wilp T, Lukas RP, Harding U, Weber TP, Van Aken H, Bohn A (2011): Beeinflussen soziodemografische Faktoren Notarzteinsätze?: Analyse in der Stadt Münster. Anaesthesist 60, 929-936

Engelhardt T, Weiss M (2012): A child with a difficult airway: what do I do next? Curr Opin Anesthesiol 25, 326-332

Evans CS, Platts-Mills TF, Fernandez AR, Grover JM, Cabanas JG, Patel MD, Vilke GM, Brice JH (2017). Repeated emergency medical services use by older adults: Analysis of a comprehensive statewide database. Ann Emerg Med 무, 506-515

Everding S, Römer S, Bohn A, Holz E, Lieder F, Baumgart P, Loyen M, Waltenberger J, Lebiedz P (2015): Clinical practice of systemic lysis in prehospital resuscitation. Success and complication rates. Med Klin Intensivmed Notfmed 110, 445-451

Eyer F, Zilker T (2012): Drogenintoxikationen. Notf Rett Med 15, 569-576

Fehn K (2014): Strafbarkeitsrisiken für Notärzte und Aufgabenträger in einem Telenotarzt-System. MedR 32, 543-552

Felzen M, Hirsch F, Brokmann JC, Rossaint R, Beckers SK (2018): Anforderungs- und Qualifikationsprofil an den Notarzt in der Telenotfallmedizin. Notf Rett Med 21, 590-597

Felzen M, Beckers SK, Brockert A -K., Follmann A, Hirsch F, Rossaint R, Schröder H (2019): Wie oft sind Notärzte an der Einsatzstelle erforderlich?. Notf Rett Med (im Druck)

Feuerwehr Göttingen (2017): Leistungen des Rettungsdienstes 2015.

https:// feuerwehr.goettingen.de/staticsite/staticsite.php?menuid=87\&topmenu=8, abgerufen am 21.01.2020

Fischer M, Krep H, Wierich D, Heister U, Hoeft A, Edwards S, Castrillo-Riesgo LG, Krafft T (2003): Comparison of the emergency medical services systems of Birmingham and Bonn: process efficacy and cost effectiveness. Anasthesiol Intensivmed Notfallmed Schmerzther $\underline{38}, 630-642$ 
Fischer M, Kamp J, Garcia-Castrillo Riesgo L, Robertson-Steel I, Overton J, Ziemann A, Krafft T (2011): Comparing emergency medical service systems-A project of the European Emergency Data (EED) Project. Resuscitation 2, 285-293

Fischer M, Kehrberger E, Marung H, Moecke H, Prückner S, Trentzsch H, Urban B, Fachexperten der Eckpunktepapier-Konsensus-Gruppe (2016): Eckpunktepapier 2016 zur notfallmedizinischen Versorgung der Bevölkerung in der Prähospitalphase und in der Klinik. Notf Rett Med 19, 387-395

Fjaeldstad A, Kirk MH, Knudsen L, Bjerring J, Christensen EF (2013): Physician-staffed emergency helicopter reduces transportation time from alarm call to highly specialized centre. Dan Med J 60, A4666

Flake F, Gliwitzky B, Grusnick HM, König MK, Beckers SK (2013): Notfallsanitäter - Chancen und Perspektiven des neuen Berufsbildes. Notf Rett Med 16, 598-603

Fleßa S (2005): Die Zukunft der Kleinst- und Kleinkrankenhäuser in Deutschland. Gesundh ökon Qual manag $\underline{10}, 295-302$

Franschman G, Andriessen TMJC, Boer C, Van der Naalt J, Horn J, Haitsma I, Vos PE (2013): Physician-based emergency medical service deployment characteristics in severe traumatic brain injury: a Dutch multicenter study. Injury $\underline{44}, 1232-1236$

Gal J, Gotthardt P, Vorstand der DGINA, Vorstand der DGSiM (2016): Klinische Notfallmedizin - Implementierung der Simulation in die Weiterbildung. Notf Rett Med 19, 491-495

Galvagno SM, Nahmias JT, Young DA (2019): Advanced Trauma Life Support ${ }^{\circledR}$ Update 2019: Management and applications for adults and special populations. Anesthesiol Clin $\underline{37}, 13-$ 32

Gomes E, Araújo R, Soares-Oliveira M, Pereira N (2004): International EMS systems: Portugal. Resuscitation $\underline{62}, 257-260$

Greiner F, Brammen D, Kulla M, Walcher F, Erdmann B (2018): Standardisierte Erhebung von Vorstellungsgründen in der Notaufnahme. Med Klin Intensivmed Notfmed $\underline{113}, 115-123$

Gretenkort P, Beneker J, Dörges V, Fischer L, Kann D, Sefrin P (2016): Strukturänderungen in der präklinischen Notfallmedizin - Standortbestimmung 2016. Notarzt 32, 264-270

Gretenkort P, Beneker J, Dörges V, Sefrin P, Fischer L, Riebandt F (2017): Einsatz von Notärzten in Ländern mit Paramedic-System. Notarzt 33, 272-278

Gretenkort P, Dörges V, Sefrin P, Beneker J, Riebandt F (2019): Zukünftige Aufgaben des Notarztes - wegweisende Aspekte aus den Nachbarländern. Notarzt $\underline{35}$, 68-75

Gries A, Helm M, Martin E (2003): Zukunft der präklinischen Notfallmedizin in Deutschland. Anaesthesist $\underline{52}, 718-724$

Gries A, Zink W, Bernhard M, Messelken M, Schlechtriemen T (2005): Einsatzrealität im Notarztdienst. Notf Rett Med $\underline{8}, 391-398$

Gries A, Sikinger M, Hainer C, Ganion N, Petersen G, Bernhard M, Schweigkofler U, Stahl P, Braun J (2008): Versorgungszeiten bei Traumapatienten im Luftrettungsdienst. Anaesthesist $\underline{57}, 562$

Gries A, Kumle B, Zimmermann M, Wilke P (2013): Zentrale Notaufnahme - Wo stehen wir heute? Notfallmed up2date $\underline{8}, 97-108$ 
Gries A, Lenz W, Stahl P, Spiess R, Luiz T (2014): On-scene times for helicopter services. Influence of central dispatch center strategy. Anaesthesist $\underline{63}, 555-562$

Gries A, Bernhard M, Helm M, Brokmann J, Gräsner JT (2017): Future of emergency medicine in Germany 2.0. Anaesthesist 66, 307-317

Gupta S, Green C, Subramaniam A, Zhen LD, Low E, Tiruvoipati R (2017): The impact of delayed rapid response call activation on patient outcomes. J Crit Care $41,86-90$

Hamilton A, Steinmetz J, Wissenberg M, Torp-Pedersen C, Lippert FK, Hove L, Lohse N (2016): Association between prehospital physician involvement and survival after out-of-hospital cardiac arrest: A Danish nationwide observational study. Resuscitation 108, 95-101

Harmsen AMK, Giannakopoulos G, Franschman G, Christiaans H, Bloemers F (2017): Limitations in prehospital communication between trauma helicopter, ambulance services, and dispatch centers. J Emerg Med 2, 504-512

Häske D, Schempf B, Gaier G, Niederberger C (2014): Prähospitale Analgosedierung durch Rettungsassistenten. Anaesthesist $\underline{63}, 209-216$

Hecker N, Domres BD (2018): The German emergency and disaster medicine and management system-history and present. Chin J Traumatol 21, 64-72

Heegaard W, Hildebrandt D, Spear D, Chason K, Nelson B, Ho J (2010): Prehospital ultrasound by paramedics: Results of field trial. Acad Emerg Med 17, 624-630

Hegenberg K, Trentzsch H, Groß S, Prückner S (2019): Use of pre-hospital emergency medical services in urban and rural municipalities over a 10year period: an observational study based on routinely collected dispatch data. Scand J Trauma Resusc Emerg Med. 27, 35

Hegenberg K, Trentzsch H, Prückner S (2019): Differences between cases admitted to hospital and discharged from the emergency department after emergency medical services transport. BMJ Open 2, e030636

Heinzel O, Eppich WJ, Schwindt JC, Hoffmann F (2011): Der lebensbedrohliche Kindernotfall im Notarztdienst. Notf Rett Med 14, 151

Helm M, Gliwitzky B, Gries A, Häske D, Hossfeld B (2017): Herausforderungen für die Zukunft der prähospitale Notfallmedizin. Notf Rett Med 20, 567-569

Helm M, Kulla M, Hossfeld B (2018): S3-Leitlinie Polytrauma - Was muss der Notarzt wissen? Notarzt $\underline{34}, 272-278$

Hennes HJ, Lang C (2000): Notarzt-Indikationskatalog. Notf Rett Med $\underline{3}$, 81-82

Heppner HJ, Wiesner R, Schuster S, Thiem U, Christ M, Singler K (2014): Bedeutung der demographischen Entwicklung für die Notfallmedizin. Notf Rett Med 17, 494-499

Hesselfeldt R, Pedersen F, Steinmetz J, Vestergaard L, Simonsen L, Jørgensen E, Clemmensen P, Rasmussen L (2013): Implementation of a physician-staffed helicopter: impact on time to primary PCI. EuroIntervention 2, 477-483

Hinkelbein J, Dambier M, Viergutz T, Genzwürker H (2008): A 6-year analysis of german emergency medical services helicopter crashes. J Trauma Acute Care Surg 64, 204-210

Hjälte L, Suserud BO, Herlitz J, Karlberg I (2007): Why are people without medical needs transported by ambulance? A study of indications for pre-hospital care. Eur J Emerg Med $\underline{14}, 151-156$ 
Holling G (2003): Krankenkraftwagen und Krankentransportmittel - Erkenntnisse 4œ Jahre nach Einführung der Europa Norm EN1789. Notfallmed 29, 422-423

Horton MA, Beamer C (2008): Powered intraosseous insertion provides safe and effective vascular access for pediatric emergency patients. Pediatr Emerg Care 24, 347-350

Igarashi Y, Yokobori S, Yamana H, Nagakura K, Hagiwara J, Masuno T, Yokota H (2018):

Overview of doctor-staffed ambulance use in Japan: a nationwide survey and 1-week study. Acute Med Surg $\underline{5}, 316-320$

Jansen G, Latka E, Mertzlufft F (2018): Der Notfallsanitäter im Ergänzungslehrgang - Analyse der Notfallsanitäter-Ergänzungsprüfungen 10/2015 bis 10/2016 an einem Simulationsmodell. Notarzt $\underline{34}, 308-315$

Jantzen T, Burgkhardt M, Burgkhardt A, Kampmann J (2008): Geschichte der Notfallmedizin im Osten Deutschlands. Notf Rett Med 11, 571-578

Kanz D: Kann der Notfallsanitäter mit seinen erweiterten Kompetenzen das Notarztsystem entlasten? Med. Diss. Charité - Berlin 2018

Karutz H (2014) Der psychosoziale Notfall - für den Rettungsdienst ein Fehleinsatz?. Rettungsd 37, 632-637.

Katzenmeier C, Schrag-Slavu S: Medizinrechtliche Fragestellungen des Einsatzes der Telemedizin im Rettungsdienst. In: Katzenmeier C, Schrag-Slavu S (Hrsg.): Rechtsfragen des Einsatzes der Telemedizin im Rettungsdienst. Springer, Berlin, Heidelberg 2010, 23-65

Kill C (2008): Versorgung des Polytraumas. Notarzt 24, 46-51

Kirchner H, Hill A (2013): Der psychiatrische Notfall. Notarzt 29, 99-105

Kleber C, Giesecke MT, Tsokos M, Haas NP, Schaser KD, Stefan P, Buschmann CT (2012):

Overall distribution of trauma-related deaths in Berlin 2010: advancement or stagnation of German trauma management? World J Surg $\underline{36}$, 2125-2130

Klier M, Wanka-Pail ER, Gehring C, Prueckner S, PrimAIR-Konsortium (2015): Luftrettung rund um die Uhr - Welchen Einfluss hat das Wetter? Notf Rett Med 18, 130-138

Klingner CM, Herdtle S, Brodoehl S, Hohenstein C, Wild T, Behringer W, Witte OW, Günther A (2017): Mit Notarzt schneller zur Lyse. Notfall Rettungsmed 20, 509-514

Klingner CM, Herdtle S, Brodoehl S, Hohenstein C, Wild T, Behringer W, Witte OW, Günther A (2018): Akuter Schlaganfall - Aufgabe für Notarzt oder Rettungsassistent? Notf Rett Med 21, 147-148

Klingshirn H (2001): Notfallmedizin in Bayern - von den Anfängen bis zum heutigen Notarztsystem*. Notarzt $\underline{17}, 186-191$

Knapp J, Bernhard M, Hainer C, Sikinger M, Brenner T, Schlechtriemen T, Gries A (2008): Besteht ein Zusammenhang zwischen der Einschätzung der Vitalgefährdung und der notfallmedizinischen Erfahrung des Notarztes? Anaesthesist 57, 1069-1074

Knapp J, Häske D, Böttiger BW, Limacher A, Stalder O, Schmid A, Schulz S, Bernhard M (2019): Influence of prehospital physician presence on survival after severe trauma: Systematic review and meta-analysis. J Trauma Acute Care Surg 87, 978-989 
Knudsen L, Stengaard C, Hansen TM, Lassen JF, Terkelsen CJ (2012): Earlier reperfusion in patients with ST-elevation myocardial infarction by use of helicopter. Scand J Trauma Resusc Emerg Med 20, 70

Koch B, Wendt M, Lackner CK, Ahnefeld FW (2008): Herausforderungen an die Notfallversorgung der Zukunft: „Regional Health Care“ (RHC). Notf Rett Med 11, 491499

Koncz V, Kohlmann T, Bielmeier S, Urban B, Prückner S (2019): Tele-emergency physician : New care concept in emergency medicine. Unfallchirurg 122, 683-689

Konrad C (1998): Learning manual skills in anesthesiology: Is there a recommended number of cases for anesthetic procedures? Anesth Analg $\underline{86}$, 635-639

Kosan J: Notarzteinsätze in Alten- und Pflegeheimen: der NAW als Lückenbüßer; eine prospektive Studie. Med. Diss. Charité - Berlin 2012

König, M.K., Flake, F., Semmel, T (2018): Schlaganfall - Lassen sich die Zeiten optimieren? Notfall Rettungsmed 21, 146

Kreimeier U, Arntz HR (2012): Jugend im Rausch. Notf Rett Med 15, 189-190

Kumpch M, Luiz T (2011): Integrierte Leitstelle als Logistikzentrale. Notf Rett Med 14, 192-196

Laatz D, Welzel T, Stassen W (2019): Developing a South African Helicopter Emergency Medical Service Activation Screen (SAHAS): A Delphi study. Afr J Emerg Med 2, 1-7

Lackner CK, Altemeyer KH (2005): Quo vadis NACA-Score? Notf Rett Med $\underline{8}$, 85-86

Landesamt für Statistik Niedersachsen (2019), Bevölkerungsveränderungen der kreisfreien Städte und Landkreise im Jahr 2019.

https:/ /www.statistik.niedersachsen.de/themenbereiche/bevoelkerung/themenbereichbevoelkerung---tabellen-87673.html, abgerufen am 20.01.2020

Lawrence RJ (2013): Urban Health Challenges in Europe. J Urban Health 90, 23-36

Lebiedz P, Radke R, Bohn A, Hilker E, Vahlhaus C (2011): Präklinische Strategien zur Optimierung der Behandlung von Patienten mit ST-Hebungsinfarkten. Intensivmed 48, 130-134

Lechleuthner A (2019): Architektur des Rettungsdienstes in Deutschland. Notf Rett Med 22, 136 146

Lechleuthner A, Neupert M (2015): Tätigkeit als Notfallsanitäter im öffentlichen Rettungsdienst. Notf Rett Med 18, 413-420

Lenz W, Luderer M, Seitz G, Lipp M (2000): Die Dispositionsqualität einer Rettungsleitstelle. Notf Rett Med $\underline{3}, 72-80$

Leszczyński P, Mioduski M, Gałązkowski R (2019): The NACA score as a predictor of ventricular cardiac arrhythmias- A retrospective six-year study. Am J Emerg Med $\underline{\text { S0735-6757, 30852-6 }}$

Lippert HD (2013): Der Notfallsanitäter - nur der Rettungsassistent in einem anderen Kleid? Notf Rett Med 16, 216-218

Lohs T (2016): Qualitätsindikatoren für den Rettungsdienst in Baden-Württemberg. Notf Rett Med $\underline{19}, 625-631$ 
Lohs T: Qualitätssicherung im Rettungsdienst Baden-Württemberg. In: Neumayr A, Baubin M, Schinnerl A (Hrsg.): Herausforderung Notfallmedizin: Innovation - Vision - Zukunft. Springer, Berlin, Heidelberg 2018, 233-243

Lossius H, Søreide E, Hotvedt R, Hapnes S, Eielsen O, Førde O, Steen P (2002): Prehospital advanced life support provided by specially trained physicians: Is there a benefit in terms of life years gained? Acta Anaesthesiol Scand 46, 771-8

Luger MF, Luger TJ (2017): Anesthesiological care of trauma patients in orthogeriatric comanagement. Anaesthesist $\underline{66}, 375-392$

Luiz T (2013): Psychosoziale Notfälle - Einsatz für den Notarzt? Notf.med up2date $\underline{8}$, 205-216

Luiz T, van Lengen RH, Wickenkamp A, Kranz T, Madler C (2011): Operational availability of ground-based emergency medical services in Rheinland-Palatinate: state-wide web-based system for collation, display and analysis. Anaesthesist $\underline{60}, 421-426$

Luiz T, Jung J, Flick S (2014): Quo vadis, preclinical emergency physician?: results of a survey of emergency medical services in Rhineland-Palatinate (Germany). Anaesthesist $\underline{63}$, 294-302

Luiz T, Scherer G, Wickenkamp A, Blaschke F, Hoffmann W, Schiffer M, Zimmer J, Schaefer S, Voigt C (2015): Prehospital analgesia by paramedics in Rhineland-Palatinate : Feasability, analgesic effectiveness and safety of intravenous paracetamol. Anaesthesist 64, 927-936

Luiz T, Kumpch M, Grüttner J, Madler C, Viergutz T (2016): Prehospital CPAP therapy by emergency physicians in patients with acute respiratory failure due to acute cardiogenic pulmonary edema or acutely exacerbated COPD. In Vivo $\underline{30}, 133-139$

Luiz T, Marung H, Pollach G, Hackstein A (2019): Implementierungsgrad der strukturierten Notrufabfrage in deutschen Leitstellen und Auswirkungen ihrer Einführung. Anaesthesist 68, 282-293

Marshall JR, Katzer R, Lotfipour S, Chakravarthy B, Shastry S, Andrusaitis J, Anderson CL, Barton ED (2017): Use of physician-in-triage model in the management of abdominal pain in an emergency department observation unit. West J Emerg Med 18, 181-188

Marung H, Blau J, Lenz W, Moecke H (2013): Dispatcher assisted CPR: Implementation of 2010 guidelines in Germany. Resuscitation 4ㅜ, S67

Maurer A, Mayr B, Kaiser H, Schinnerl A, Baubin M (2015): Leitstelle Tirol: Möglichkeiten der standardisierten Notrufabfrage mit dem Medical Priority Dispatch System(C). Notf Rett Med 18, 560-566

Meißner W von (2017): Kindernotfälle - Eine besondere Herausforderung im Rettungsdienst. Retten! $\underline{6}, 173-173$

Messelken M, Dirks B (2001): Zentrale Auswertung von Notarzteinsätzen im Rahmen externer Qualitätssicherung. Notf Rett Med 4, 408-415

Messelken M, Schlechtriemen Th (2003): Der minimale Notarztdatensatz MIND2. Notf Rett Med 6, 189-192

Messelken M, Schlechtriemen T, Arntz HR, Bohn A, Bradschetl G, Brammen D, Braun J, Gries A, Helm M, Kill C, et al. (2011): Der Minimale Notfalldatensatz MIND3. Notarzt 27, 197202 
Metelmann C, Metelmann B, Scheer C, Gründling M, Henkel B, Hahnenkamp K, Brinkrolf P (2018): Sepsis detection in emergency medicine : Results of an interprofessional survey on sepsis detection in prehospital emergency medicine and emergency departments. Anaesthesist 67, 584-591

Mikkelsen S, Krüger AJ, Zwisler ST, Brøchner AC (2015): Outcome following physician supervised prehospital resuscitation: a retrospective study. BMJ Open $\underline{5}$, e006167

Miró Ò (2010): State of emergency medicine in Spain. Int J Emerg Med $\underline{3}$, 219-226

Moecke H, Ahnefeld FW (1997): Qualitätsmanagement in der Notfallmedizin. Anaesthesist $\underline{46}$, $787-800$

Moecke H, Knobelsdorff G von (2008): The anesthesiologist in prehospital and hospital emergency medicine. Curr Opin Anaesthesiol 21, 228-232

Moecke H, Dirks B, Friedrich HJ, Hennes HJ, Lackner CK, Messelken M, Neumann C, Pajonk FG, Reng M, Ruppert M, et al. (2004): DIVI-Notarzteinsatzprotokoll. Notf Rett Med 7 , 259-261

Mürdel F: Primäre versus sekundäre Notarzteinsätze - eine Qualitätsanalyse des Rettungsdienstwesens im Einsatzgebiet Ulm. Med. Diss Ulm 2015

Neupert M, Sarangi F, e.V für die AR (2014): Stellungnahme der Arbeitsgemeinschaft RettungsdienstRecht e.V. zum „Pyramidenprozess“ zur Umsetzung des Notfallsanitätergesetzes (NotSanG). Notarzt $\underline{30}, 118-120$

Ocker H, Schörnig W, Sauer C, Hüppe M, Dörges V, Gerlach K (2006): Ambulante Patientenversorgung durch den Notarzt: Qualifikationsmerkmal oder nur Fehleinsatz? Anaesth Intensivmed 47, 124-132

Ohr T (2005): Wie verbindlich ist die Stellungnahme der Bundesärztekammer zur Notkompetenz? Notf Rett Med $\underline{8}, 440-443$

Oramary D, Al-Sadi O, Blum S, Schaser KD, Kleber C (2019): Präklinische Versorgung des Polytraumas. OP-Journal $\underline{36}, 5-16$

Osterloh F (2019): Notfallversorgung: Wege zu mehr Patientensteuerung. Dtsch Arztebl 116, A6163

Pajonk FGB (2015): Zur Situation der Notfall- und Akutpsychiatrie in Deutschland. Nervenarzt $\underline{86}$, $1081-1090$

Pfeifer R, Tarkin IS, Rocos B, Pape HC (2009): Patterns of mortality and causes of death in polytrauma patients-has anything changed? Injury $\underline{40}, 907-911$

Pfütsch P (2019): From volunteer work to critical care paramedic : The history of an ongoing process of professionalisation. Med Klin Intensivmed Notfmed 114, 258-262

Pietschmann H (2001) Ist Rettungswagenfahren lebensgefährlich? Rettungsdienst 24, 22-25

Prause G, Wildner G, Kainz J, Bößner T, Gemes G, Dacar D, Magerl S (2007): Strategien zur Optimierung notärztlicher Kompetenz in der Flugrettung. Anaesthesist $\underline{56}, 461-465$

Prause G, Wildner G, Gemes G, Zoidl Ph, Zajic P, Kainz J, Pock M, Trimmel H (2017): Abgestufte präklinische Notfallversorgung - Modell Graz. Notf Rett Med 20, 501-508 
Prückner S, Luiz T, Steinbach-Nordmann S, Nehmer J, Danner K, Madler C (2008):

Notfallmedizin - Medizin für eine alternde Gesellschaft. Anaesthesist 57, 391-396

Raatiniemi L, Mikkelsen K, Fredriksen K, Wisborg T (2013): Do pre-hospital anaesthesiologists reliably predict mortality using the NACA severity score? A retrospective cohort study: Pre-hospital NACA severity score. Acta Anaesthesiol Scand 57, 1253-1259

Rasmussen MB, Frost L, Stengaard C, Brorholt-Petersen JU, Dodt KK, Søndergaard HM, Terkelsen CJ (2014): Diagnostic performance and system delay using telemedicine for prehospital diagnosis in triaging and treatment of STEMI. Heart $\underline{100}, 711-715$

Rau R, Mensing M, Brand H (2006): "Medical Emergency Services as Seen by Consumers". Health services research by CATI survey of the community. Med Klin 101, 37-47

Reifferscheid F (2019): Stellungnahme der BAND zum Indikationskatalog für den Notarzteinsatz. Notarzt $\underline{35}, 193-193$

Reifferscheid F, Harding U (2017): Einheitliche Notarzt-qualifikation: Wie steht es 13 Jahre nach der Einführung um die Zusatzweiterbildung Notfallmedizin? Anaesthesiol Intensivmed $\underline{58}$, 44-51

Reifferscheid F, Dörges V, Knacke P, Wirtz S (2010): Weiterbildungsbedingungen für Notärzte. Notarzt 26, 103-107

Reifferscheid F, Harding U, Hossfeld B (2016): Einmal Notarzt - immer Notarzt? Notf Rett Med $\underline{19}, 554-558$

Reiner-Deitemyer V, Teuschl Y, Matz K, Reiter M, Eckhardt R, Seyfang L, Tatschl C, Brainin M (2011): Helicopter transport of stroke patients and its influence on thrombolysis rates. Stroke $\underline{42}, 1295-1300$

Rezar R, Sandner G, Heschl S, Hallmann B, Prause G, Gemes G (2017): Vorteile der Hubschrauberrettung am Beispiel eines österreichischen Notarzthubschraubers. Notf Rett Med 20, 495-500

Roberts K, Blethyn K, Foreman M, Bleetman A (2009): Influence of air ambulance doctors on onscene times, clinical interventions, decision-making and independent paramedic practice. Emerg Med J 26, 128-134

Roessler M, Zuzan O (2006): EMS systems in Germany. Resuscitation 요, 45-49

Roessler M (2019a): Historischer Rückblick zur Entwicklung der Notfallmedizin in Göttingen. https:/ / ains.umg.eu/de/ueber-uns/unsere-klinik/notfallmedizin/geschichte/, abgerufen am 21.01.2020

Roessler M (2019b): Notfallmedizin heute: Rettungsdienst in Stadt und Landkreis Göttingen. https:/ / ains.umg.eu/de/ueber-uns/unsere-klinik/notfallmedizin/notfallmedizin-heute/, abgerufen am 21.01.2020

Rognås L, Hansen TM, Kirkegaard H, Tønnesen E (2013): Pre-hospital advanced airway management by experienced anaesthesiologists: a prospective descriptive study. Scand J Trauma Resusc Emerg Med 21, 58

Röhrig R, Walcher F (2014): Medizinische Dokumentation - Antike und Moderne. Notf Rett Med $\underline{17}, 650-651$ 
Rossaint R, Wolff J, Lapp N, Hirsch F, Bergrath S, Beckers SK, Czaplik M, Brokmann JC (2017): Indikationen und Grenzen des Telenotarztsystems. Notf Rett Med 20, 410-417

Rostykus P, Kennel J, Adair K, Fillinger M, Palmberg R, Quinn A, Ripley J, Daya M (2016): Variability in the treatment of prehospital hypoglycemia: A structured review of EMS protocols in the United States. Prehosp Emerg Care 20, 524-530

Roudsari BS, Nathens AB, Arreola-Risa C, Cameron P, Civil I, Grigoriou G, Gruen RL, Koepsell TD, Lecky FE, Lefering RL, et al. (2007): Emergency Medical Service (EMS) systems in developed and developing countries. Injury $\underline{38}, 1001-1013$

Ruskin K (2019): Helicopter air ambulance services. Curr Opin Anaesthesiol 32, 252-256

Savage ML, Poon KKC, Johnston EM, Raffel OC, Incani A, Bryant J, Rashford S, Pincus M, Walters DL (2014): Pre-hospital ambulance notification and initiation of treatment of ST elevation myocardial infarction is associated with significant reduction in door-to-balloon time for primary PCI. Heart Lung Circ $\underline{23}$, 435-443

Schaller, S. J., Kappler, F. P., Hofberger, C., Sattler, J., Wagner, R., Schneider, G., Blobner, M., \& Kanz, K. G. (2019). Differences in pain treatment between surgeons and anaesthesiologists in a physician staffed prehospital emergency medical service: a retrospective cohort analysis. BMC anesthesiol $\underline{19}, 18$.

Schellhaaß A, Popp E (2014): Air rescue: current significance and practical issues. Anaesthesist 63 , 971-980

Schempf B, Casu S, Häske D (2017): Prehospital analgesia by emergency physicians and paramedics : Comparison of effectiveness. Anaesthesist 66 , 325-332

Schlechtriemen T, Burghofer K, Stolpe E, Altemeyer KH, Lackner CK (2005a): Der Münchner NACA-Score: Eine Modifikation des NACA-Score für die präklinische Notfallmedizin. Notf Rett Med $\underline{8}, 109-111$

Schlechtriemen T, Burghofer K, Lackner CK, Altemeyer KH (2005b): Validierung des NACAScore anhand objektivierbarer Parameter. Notf Rett Med $\underline{8}, 96-108$

Schlechtriemen T, Masson R, Burghofer K, Lackner CK, Altemeyer KH (2006): Pädiatrische Notfälle in der präklinischen Notfallmedizin. Anaesthesist $\underline{55}, 255-262$

Schlechtriemen T, Dirks B, Lackner CK, Moecke H, Stratmann D, Krieter H, Altemeyer KH (2007): Leitstelle - Perspektiven für die zentrale Schaltstelle des Rettungsdienstes. Notf Rett Med 10, 47-57

Schlechtriemen T, Armbruster W, Adler J, Bartha C, Becker K, Höhn M, Kubulus D, Morbe A, Reeb R, Schmidt D, et al. (2014): Herausforderung Notarztdienst. Notf Rett Med 17, 39_ 45

Schmiedel R, Behrendt H: Analyse des Leistungsniveaus im Rettungsdienst für die Jahre 2012 und 2013. In: Bundesanstalt für Straßenwesen (Hrsg) Leistungen des Rettungsdienstes 2004/05. Berichte der Bundesanstalt für Straßenwesen, Mensch und Sicherheit 260, Fachverlag NW, Bremen 2015

Schmiedhofer MH, Searle J, Slagman A, Möckel M (2017): Inanspruchnahme zentraler Notaufnahmen: Qualitative Erhebung der Motivation von Patientinnen und Patienten mit nichtdringlichem Behandlungsbedarf. Gesundheitswesen $\underline{79}$, 835-844 
Schmitt TK, Luiz T, Poloczek S, Huber T, Kumpch M, Madler C (2002): Sozialepidemiologie einer neuen Einsatzkategorie. Notf Rett Med $\underline{5}, 102-109$

Schneider F, Martin J, Schneider G, Schulz CM (2018): The impact of the patient's initial NACA score on subjective and physiological indicators of workload during pre-hospital emergency care. PLoS ONE $\underline{13}$, e0202215

Schulz-Stubner S (2003): Bring the Doctor to the Patient: the German Notarzt System. Anesth Analg 96, 912

Schuster M, Pints M, Fiege M (2010): Duration of mission time in prehospital emergency medicine: effects of emergency severity and physicians level of education. Emerg Med J 27, 398-403

Schuster S, Singler K, Dormann H (2016): Geriatrische Notfallpatienten. Notf Rett Med 19, $657-$ 665

Schweigkofler U, Braun J, Schlechtriemen T, Hoffmann R, Lefering R, Reimertz C (2015): Significance of helicopter emergency medical service in prehospital trauma care. Z Orthop Unfall $\underline{153}, 387-391$

Sefrin P (2003): History of the rescue service in Germany - especially in regard to emergency medicine. Anasthesiol Intensivmed Notfallmed Schmerzther 38, 623-629

Sefrin P (2009): Kindernotfälle - Eine besondere Herausforderung für den Notarzt. Notarzt $\underline{25}$, 107-108

Sefrin P (2012): Die präklinische Notfallversorgung von schwerverletzten, polytraumatisierten Patienten auf der Basis der S3-Polytrauma-Leitlinie. Notarzt 28, 66-70

Sefrin P (2013): Development of emergency medical services in Germany - Western part of Germany. Anasthesiol Intensivmed Notfallmed Schmerzther 48, 734-738

Sefrin P, Kuhnigk H (2006): The position of the emergency physician in the emergency medical service. Anasthesiol Intensivmed Notfallmed Schmerzther $\underline{41}, 2-8$

Sefrin P, Ripberger G (2008): Stellenwert des Notarztes im Rahmen der Bewältigung psychosozialer Probleme. Intensivmed 45, 55-63

Sefrin P, Berger HJ, Schlennert B, Reindl K (2007): Die „Rückmeldezahl” als Basis eines Qualitätsmanagements im Rettungsdienst in Bayern. Notarzt 23, 195-200

Sikinger M, Bernhard M, Bujard M, Serf C, Sacconi T, Hillger K, Gries A (2005): Notfallmedizin gestern, heute und morgen. Notf Rett Med $\underline{8}, 133-138$

Soar J, Nolan JP, Böttiger BW, Perkins GD, Lott C, Carli P, Pellis T, Sandroni C, Skrifvars MB, Smith GB, et al. (2015): European Resuscitation Council Guidelines for Resuscitation 2015: Section 3. Adult advanced life support. Resuscitation 95, 100-147

Soda H, Ziegler V, Shammas L, Griewing B, Kippnich U, Keidel M, Rashid A (2017): Telemedizinische Voranmeldung in der akuten Schlaganfallversorgung. Nervenarzt $\underline{88}$, 120-129

Sollid SJM, Rehn M (2017): The role of the anaesthesiologist in air ambulance medicine. Curr Opin Anaesthesiol $\underline{30}$, 513-517

Sørensen JT, Terkelsen CJ, Steengaard C, Lassen, J. F., Trautner, S., Christensen, E. F., Nielsen, T. T., Bøtker, H. E., Andersen, H. R., Thygesen, K. (2011): Prehospital troponin T testing in 
the diagnosis and triage of patients with suspected acute myocardial infarction. Am J Cardiol 107, 1436-1440.

Statistisches Bundesamt (Destatis) (Hrsg.): Bevölkerung Deutschlands bis 2060. Statistisches Bundesamt, Wiesbaden 2019. https://www.destatis.de/DE/Themen/GesellschaftUmwelt/Bevoelkerung/Bevoelkerungsvorausberechnung/Publikationen/DownloadsVorausberechnung/bevoelkerung-deutschland-2060-

5124202199004.pdf?_blob=publicationFile, abgerufen am: 12.12.2019

Staufer A (2018): Recht: Nebenbei Notarzt? Was es dann zu beachten gilt! Dtsch Arztebl 115, 2-4

Stengaard C, Sørensen JT, Rasmussen MB, Bøtker MT, Pedersen CK, Terkelsen CJ (2016): Prehospital diagnosis of patients with acute myocardial infarction. Diagnosis $\underline{3}, 155-166$

Stevanovic A, Beckers SK, Czaplik M, Bergrath S, Coburn M, Brokmann JC, Hilgers RD, Rossaint R, (2017): Telemedical support for prehospital Emergency Medical Service (TEMS trial): study protocol for a randomized controlled trial. Trials $\underline{18}, 43$

Stewart J, Rerucha C, Galfand W (2019): Does transport by helicopter compared with ground ambulance improve mortality for major trauma? Evidence-based Practice 22, 17-18

Stolpe E, Schrödel M, Lackner ChrK, Gauss C, Klanner W (2002): Einzelversuch zur Untersuchung des Crashverhaltens von Rettungswagen (RTW/NAW). Notf Rett Med $\underline{5}$, $519-524$

Strohm PC, Zwingmann J, Bayer J, Neumann MV, Lefering R, Schmal H, Reising K (2018): Differences in the outcome of seriously injured children depending on treatment level. Unfallchirurg 121, 306-312

Studnek JR, Infinger A, Wilson H, Niess G, Jackson P, Swanson D (2018): Decreased time from 91-1 call to PCI among patients experiencing STEMI Results in a decreased one year mortality. Prehosp Emerg Care 22, 669-675

Suryanto, Plummer V, Boyle M (2017): EMS systems in lower-middle income countries: A literature review. Prehosp Disaster Med $\underline{32}, 64-70$

Süss R, Dewenter C, Ekinci A, Laslo T, Fleßa S (2020): Das Telenotarztsystem - Potentiale für die präklinische Notfallversorgung im ländlichen Raum. Gesundh ökon Qual manag (im Druck)

Takeuchi I, Fujita H, Yanagisawa T, Sato N, Mizutani T, Hattori J, Asakuma S, Yamaya T, Inagaki T, Kataoka Y, et al. (2015): Impact of doctor car with mobile cloud ECG in reducing doorto- balloon time of japanese ST-elevation myocardial infarction patients. Int Heart J $\underline{56}$, $170-173$

Tanaka K, Lorenzo RAD (2019): Lights and siren: A risky business? Ann Emerg Med 74, 110-111

Tanguay A, Lebon J, Lau L, Hébert D, Bégin F (2018): Detection of STEMI using prehospital serial 12-lead electrocardiograms. Prehosp Emerg Care 22, 419-426

Thümmler C (2003): Notfallmedizin: Senioren verändern das Einsatzspektrum. Dtsch Arztebl 100, A680

Tiesmeier J, Hilgefort S, Ueckermann FD, Jakob T, Emmerich M, Henzler D (2016): Mit welchem Spektrum und Maßnahmen muss der Notarzt bei präklinischen Kindernotfällen rechnen? Notarzt $\underline{32}, 222-227$ 
Timmermann A, Russo SG, Hollmann MW (2008): Paramedic versus emergency physician emergency medical service: role of the anaesthesiologist and the European versus the Anglo-American concept. Curr Opin Anaesthesiol 21, 222-227

Touray S, Sanyang B, Zandrow G, Touray I (2018): Incidence and outcomes after out-of-hospital medical emergencies in Gambia: A case for the integration of prehospital care and emergency medical services in primary health care. Prehosp Disaster Med $\underline{33}$, 650-657

Treffer D, Weißleder A, Helm M, Gässler H (2017): Mechanische Thoraxkompressionsgeräte. Notarzt $\underline{33}, 190-196$

Tsuchiya A, Tsutsumi Y, Yasunaga H (2016): Outcomes after helicopter versus ground emergency medical services for major trauma-propensity score and instrumental variable analyses: a retrospective nationwide cohort study. Scand J Trauma Resusc Emerg Med 24, 140

Vanni D, Palasciano G, Vanni P, Vanni S, Guerin E (2018): Medical doctors and the foundation of the International Red Cross. Intern Emerg Med 13, 301-305

von Kaufmann F, Kanz KG (2012): Die Rolle der Leitstelle im Prozess der präklinischen Versorgung. Notf Rett Med 15, 289-299

Watanabe BL, Patterson GS, Kempema JM, Magallanes O, Brown LH (2019): Is use of warning lights and sirens associated with increased risk of ambulance crashes? A contemporary analysis using national EMS information system (NEMSIS) Data. Ann Emerg Med 74, 101-109

Weiss M, Bernoulli L, Zollinger A (2001): Der NACA-Index: Aussagekraft und Stellenwert des modifizierten NACA-Indexes in der präklinischen Schweregraderfassung von Unfallpatienten. Anaesthesist $\underline{50}, 150-154$

Wenzel RR, Slany J, Weber T, Zweiker R, Watschinger W (2014): Hypertensiver Notfall hypertensive Entgleisung (Krise). Journal für Hypertonie 18, 97-100

Wigman LD, van Lieshout EMM, de Ronde G, Patka P, Schipper IB (2011): Trauma-related dispatch criteria for Helicopter Emergency Medical Services in Europe. Injury 42, 525-533

Wilhelm W (2003): Psychiatric emergencies: emergencies for the emergency physician?. Anaesthesist $\underline{52}, 575-576$

Wilson MH, Habig K, Wright C, Hughes A, Davies G, Imray CHE (2015): Pre-hospital emergency medicine. The Lancet 386, 2526-2534

Winburn AS, Brixey JJ, Langabeer J, Champagne-Langabeer T (2018): A systematic review of prehospital telehealth utilization. J Telemed Telecare 24, 473-481

Wooldridge A, Carayon P, Hoonakker P (2019): Complexity of the pediatric trauma care process: Implications for multi-level awareness. Cogn Technol Work 21, 397-416

Wutti C (2014): Der ältere Mensch als Notfallpatient. Notf Rett Med 17, 488-493

Zakariassen E, Østerås Ø, Nystøyl DS, Breidablik HJ, Solheim E, Brattebø G, Ellensen VS, Hoff JM, Hordnes K, Aksnes A, et al. (2019): Loss of life years due to unavailable helicopter emergency medical service: a single base study from a rural area of Norway. Scand J Prim Health Care 37, 233-241

Zdrenka G (2010): Der Ärztliche Bereitschaftsdienst in Bayern. Notf Rett Med 13, 114-118 


\section{Danksagung}

Für die Betreuung in Rahmen dieser Dissertation sowie für die ausgezeichneten gelehrten Kompetenzen in Rahmen meiner Weiterbildung zum Facharzt, bedanke ich mich zunächst bei PD Dr. Daniel Heise ganz herzlich.

Meine besondere Danksagung an Frau Prof. Dr. Sabine Blaschke für die großzügige Begutachtung, sowie an PD Dr. Markus Roessler und Prof. Michael Quintel für die Unterstützung in der Klinik für Anästhesiologie bei der Durchführung dieses Projektes.

Danke an den notärztlichen Kollegen der Universitätsmedizin Göttingen für die Kooperation bei der Datensammlung sowie an den Kollegen der Feuerwehr und des Rettungsdienstes Göttingen für die Zusammenarbeit.

Danke an Graciela Arce und Roberto Arnoldt für ihre uneingeschränkte Unterstützung und Motivation vom Beginn bis zum Ende dieser Arbeit.

Herzlichen Dank an Anne Arnoldt, Ute und Norbert Kaufmann für die Hilfe bei der Korrektur. 
